\title{
Formal Total Synthesis of (-)-Salicylihalamides A and B From D-Glucose and L-Rhamnose
}

\author{
Torsten Haack, KyoungLang Haack, Wibke E. Diederich, Burchelle Blackman, Subho Roy, \\ Srinivas Pusuluri, and Gunda I. Georg* \\ Department of Medicinal Chemistry, Center for Cancer Experimental Therapeutics and \\ Center for Drug Discovery, Higuchi Biosciences Center, \\ University of Kansas, 1251 Wescoe Hall Drive, \\ Lawrence, KS 66045-7582, USA
}

georg@ku.edu

\section{SUPPORTING INFORMATION}

\section{$\underline{\text { Table of contents }}$}

Proton NMR of compound 8

Carbon NMR of compound 8

Proton NMR of compound 9

Carbon NMR of compound $\mathbf{9}$

Proton NMR of compound $\mathbf{1 0}$

Carbon NMR of compound $\mathbf{1 0}$

Proton NMR of compound $\mathbf{1 1}$
S4

S5

S6

S7

S8

S9

S10
Proton NMR of compound $32 \ldots \ldots \ldots \ldots \ldots \ldots \ldots \ldots \ldots \ldots . \quad$ S48

Carbon NMR of compound $32 \ldots \ldots \ldots \ldots \ldots \ldots \ldots \ldots \ldots$ S49

Proton NMR of compound $33 \ldots \ldots \ldots \ldots \ldots \ldots \ldots \ldots \ldots \ldots . \quad$ S50

Carbon NMR of compound 33 ........................ S51

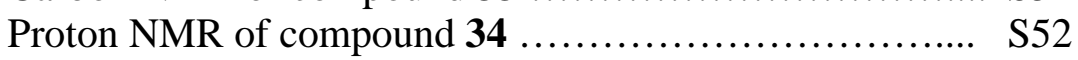

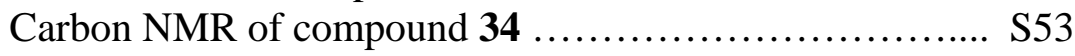

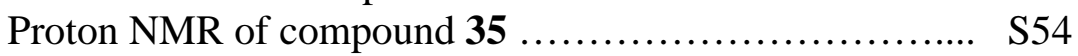


Carbon NMR of compound $\mathbf{1 1}$

Proton NMR of compound 12

Carbon NMR of compound 12

Proton NMR of compound 14 .

Carbon NMR of compound 14

Proton NMR of compound $\mathbf{1 5}$

Carbon NMR of compound $\mathbf{1 5}$

Proton NMR of compound $\mathbf{1 6}$

Carbon NMR of compound $\mathbf{1 6}$

Proton NMR of compound 17

Carbon NMR of compound $\mathbf{1 7}$

Proton NMR of compound $\mathbf{1 8}$

Carbon NMR of compound $\mathbf{1 8}$

Proton NMR of compound 19

Proton NMR of compound 20

Carbon NMR of compound 20

Proton NMR of compound $\mathbf{2 1}$

Proton NMR / HPLC data of compound 21

Carbon NMR of compound 21

Proton NMR of compound 22

Carbon NMR of compound 22

Proton NMR of compound 23

Carbon NMR of compound 23

Proton NMR of compound 25

Carbon NMR of compound 25

Proton NMR of compound $\mathbf{2 6}$

Carbon NMR of compound $\mathbf{2 6}$

Proton NMR of compound 27

Carbon NMR of compound 27

Proton NMR of compound $\mathbf{2 8}$
S11

S12

S13

S14

S15

$\mathrm{S} 16$

S17

S18

S19

S20

$\mathrm{S} 21$

S22

$\mathrm{S} 23$

S24

S25

S26

S27

S28

S29

S30

S31

S32

S33

S34

S35

S36

S37

S38

S39

S40
Carbon NMR of compound 35 .......................... S55

Proton NMR of compound 36 ........................ S56

Carbon NMR of compound 36 ......................... S57

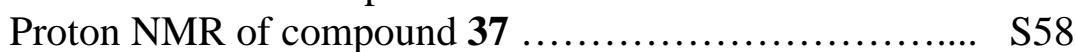

Carbon NMR of compound 37 ........................ S59

Proton NMR / HPLC data of compound 38 ................. S60

Proton NMR / HPLC data of compound 39 ................. S61

Proton NMR / HPLC data of compound 40 ................. S62

Proton NMR / HPLC data of compound $\mathbf{4 1}$................ S63

Proton NMR / HPLC data of compound 42 ................ S64

Proton NMR / HPLC data of compound 43 ................. S65

Proton NMR / HPLC data of compound 44 ................ S66

Proton NMR / HPLC data of compound 45 ................ S67

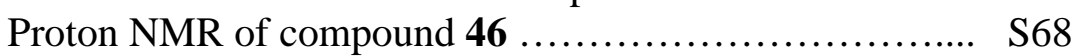

Carbon NMR of compound 46 ........................ S69

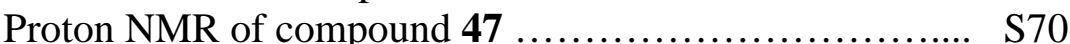

Carbon NMR of compound 47 ......................... S71

Proton NMR of compound 48 .......................... S72

Carbon NMR of compound $\mathbf{4 8}$.......................... S73

Proton NMR of compound 49alpha....................... S74

Carbon NMR of compound 49alpha....................... S75

Proton NMR of compound 49beta...................... S76

Carbon NMR of compound 49beta...................... S77

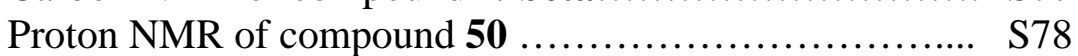

Carbon NMR of compound 50 ......................... S79

Proton NMR of compound $51 \ldots \ldots \ldots \ldots \ldots \ldots \ldots \ldots \ldots . . . \ldots \ldots$

Carbon NMR of compound 51 .......................... S81

Proton NMR of compound $52 \ldots \ldots \ldots \ldots \ldots \ldots \ldots \ldots \ldots . . . \ldots 28$

Carbon NMR of compound 52 .......................... S83

Proton NMR of compound 53 ....................... S84 
Carbon NMR of compound $\mathbf{2 8}$

Proton NMR of compound $\mathbf{2 9}$

Carbon NMR of compound $\mathbf{2 9}$

Proton NMR of compound $\mathbf{3 0}$

Carbon NMR of compound 30

Proton NMR of compound $\mathbf{3 1}$

Carbon NMR of compound 31
Carbon NMR of compound 53 ...

Carbon NMR of compound $\boldsymbol{E}-54$

Proton NMR of compound $\boldsymbol{E}-54$

S85

Carbon NMR of compound $Z-54$

Proton NMR of compound $Z-54$ 


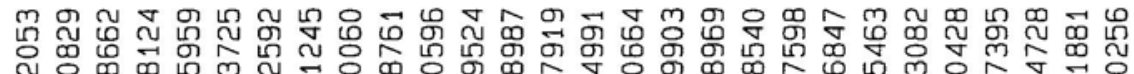

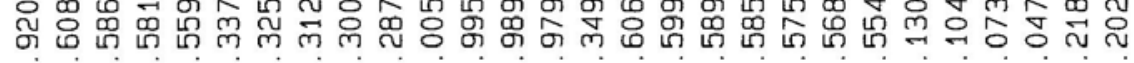

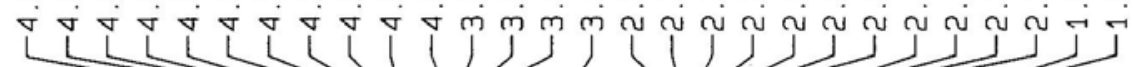
(1)

$\mathrm{HO}$

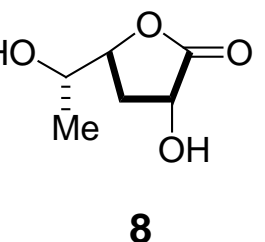

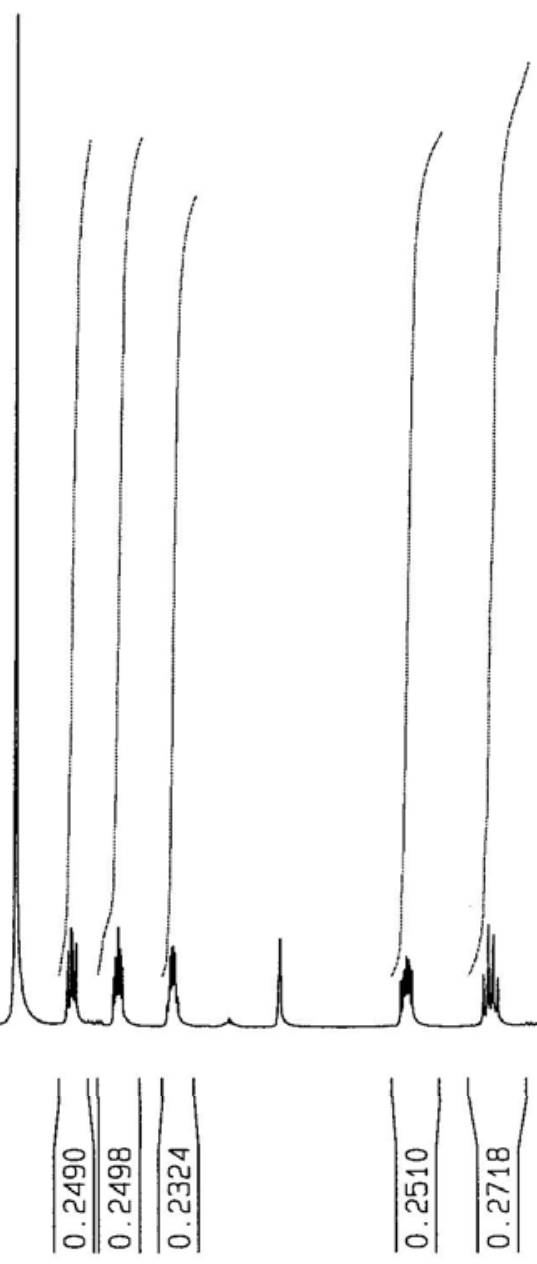



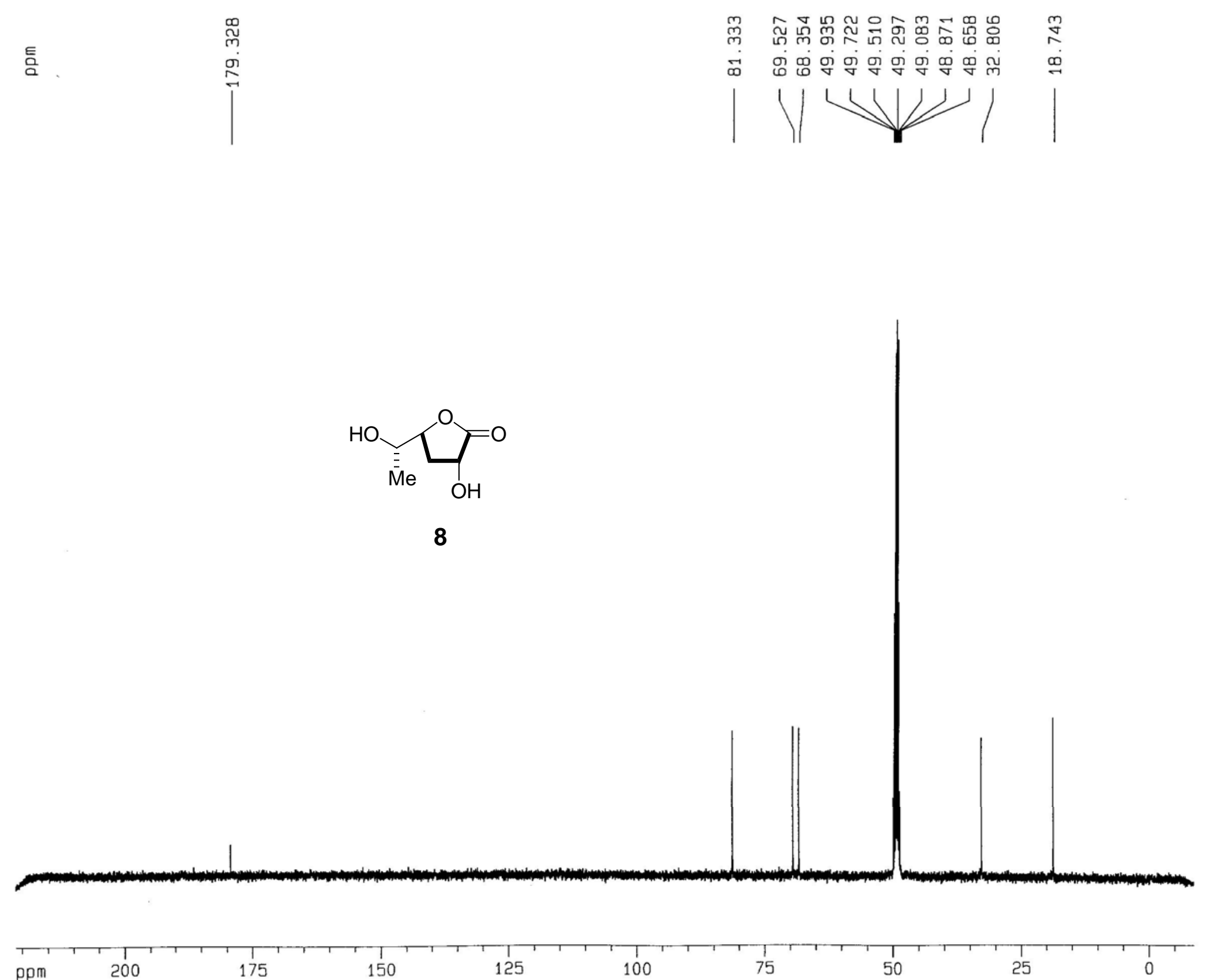


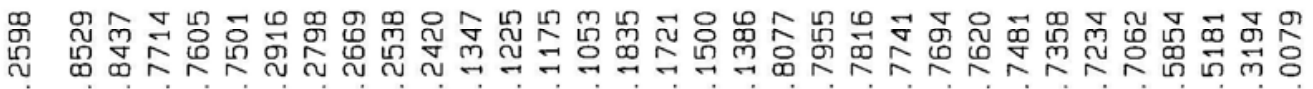

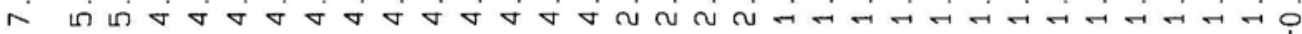
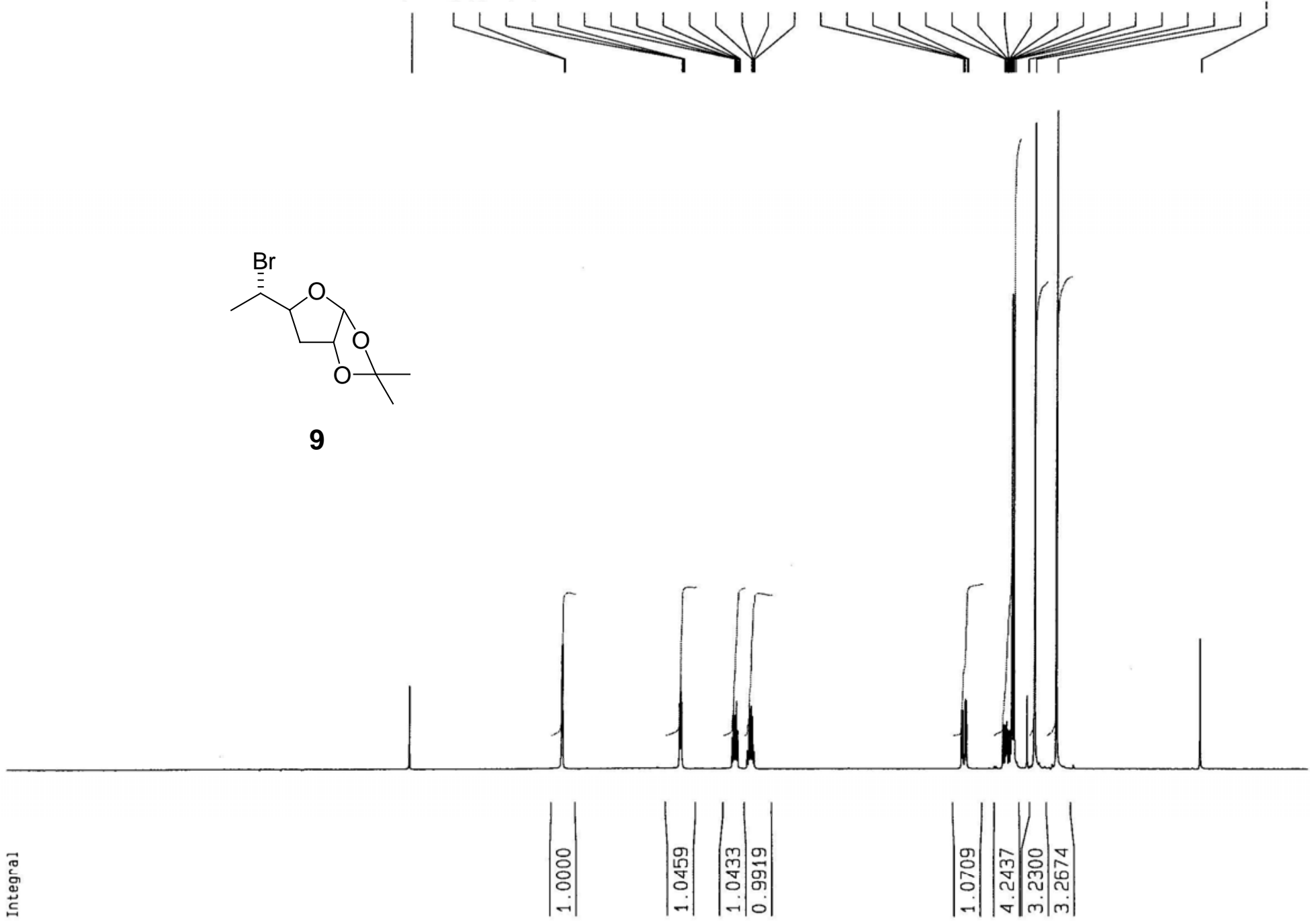

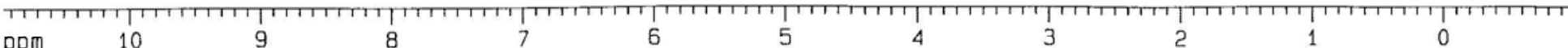




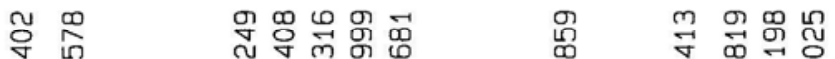

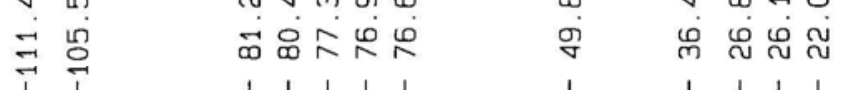

U.

$1 \mid 1$

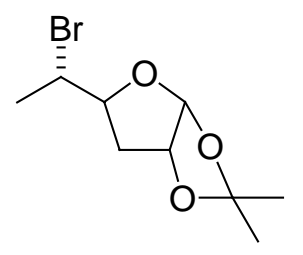

9

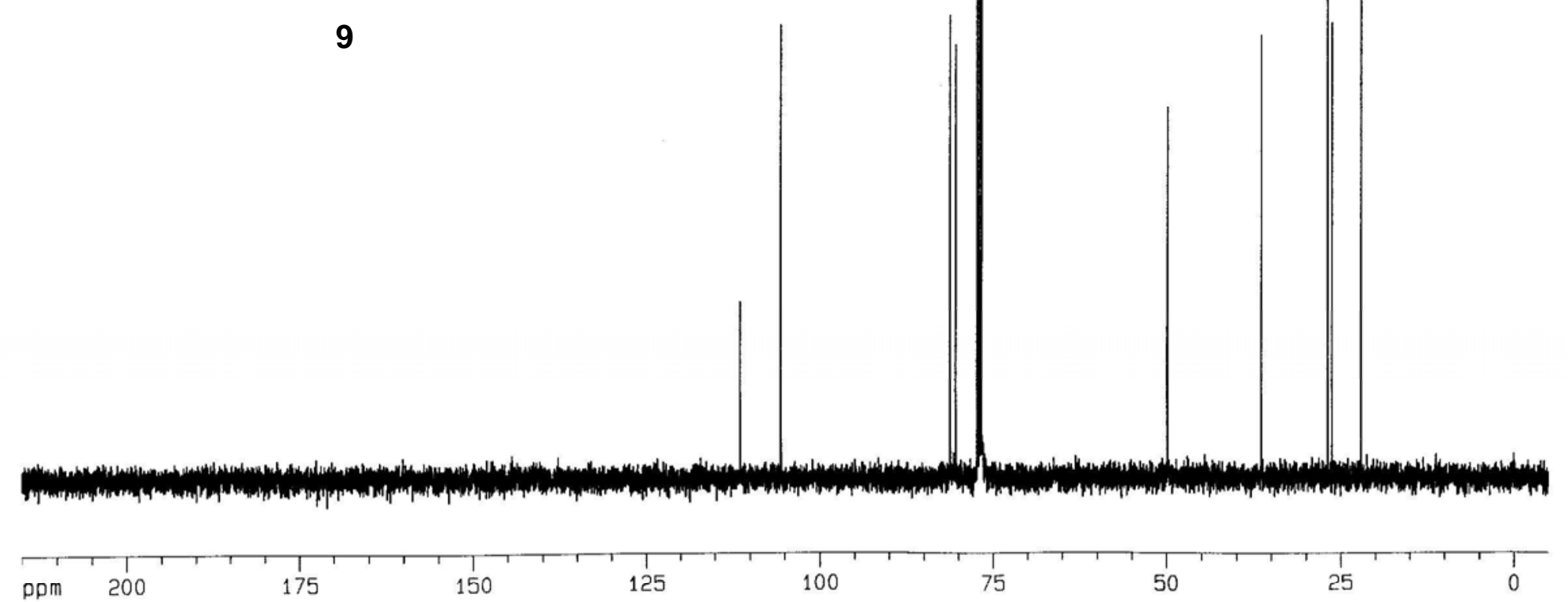



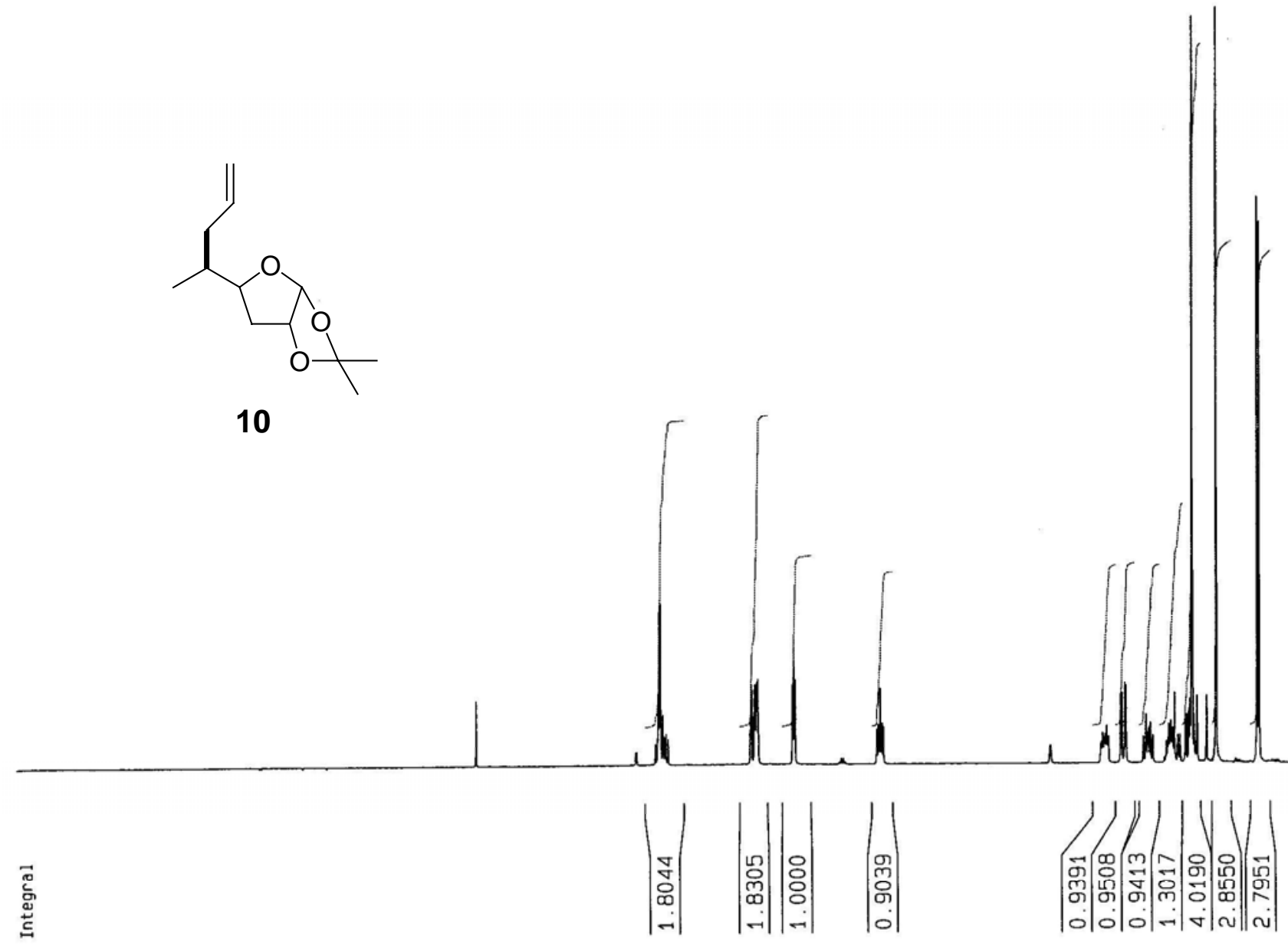


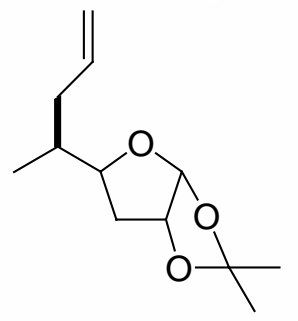

10
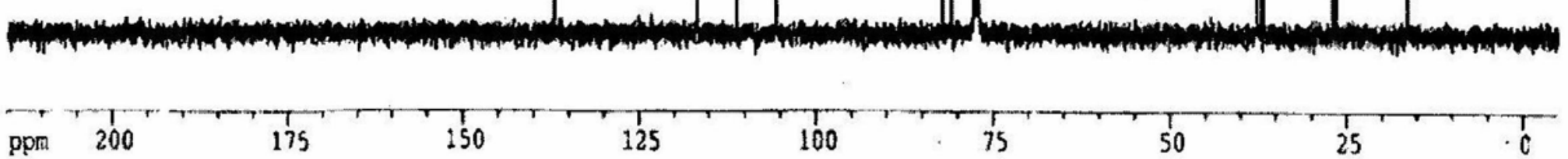


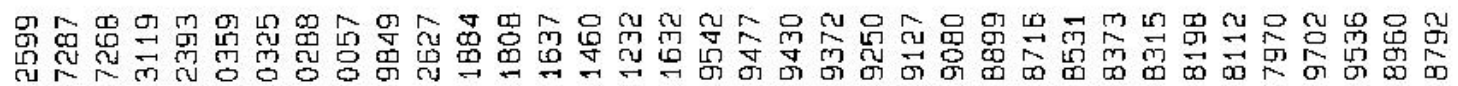

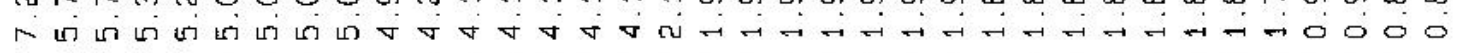
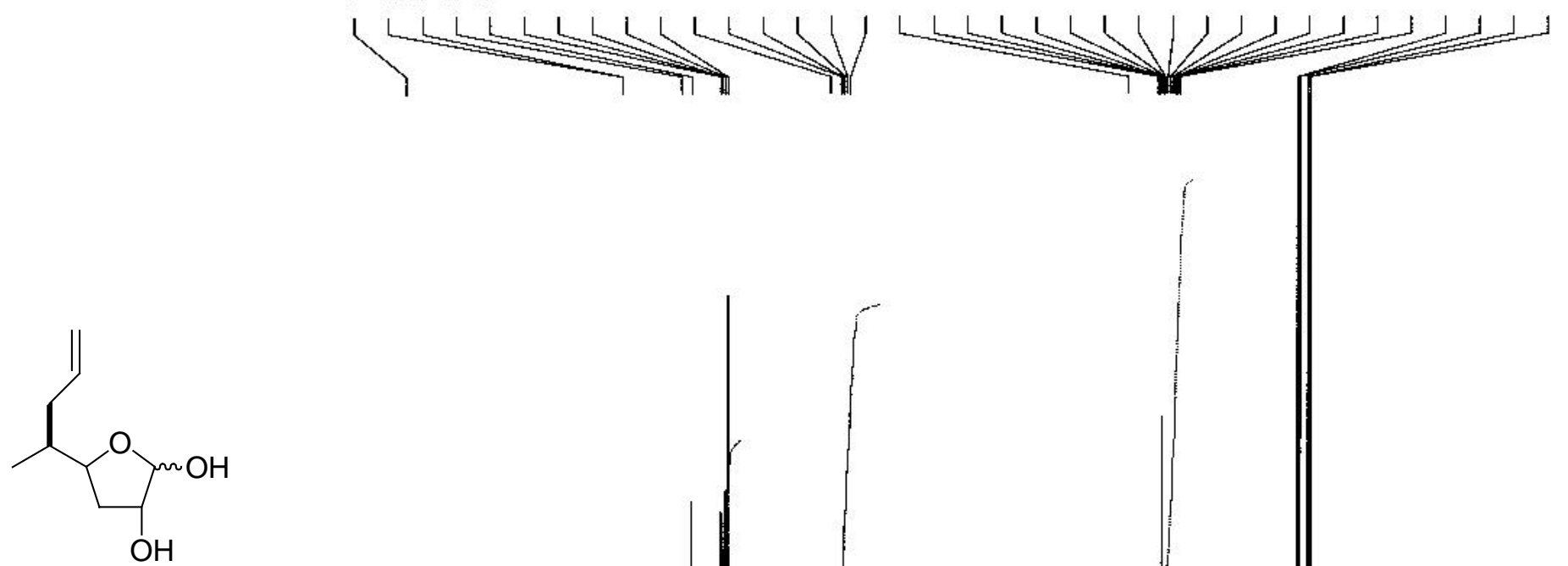

11

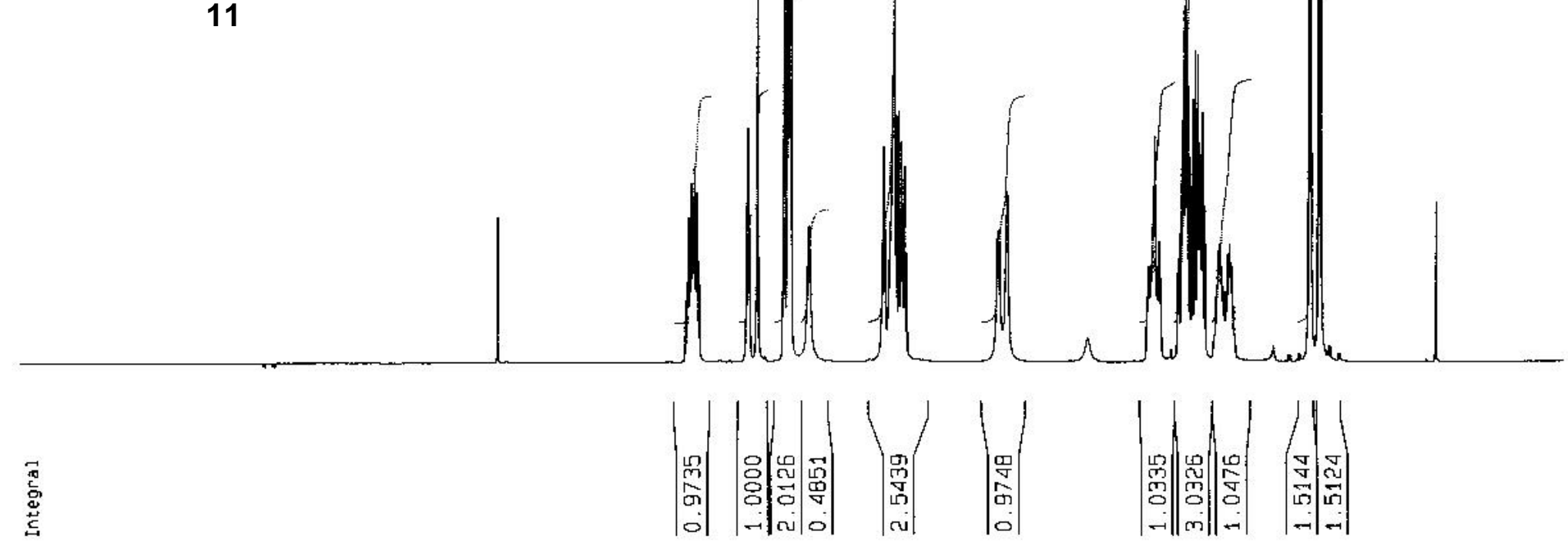




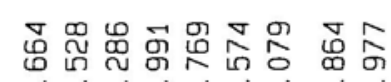

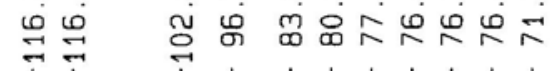

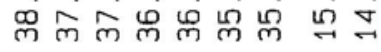
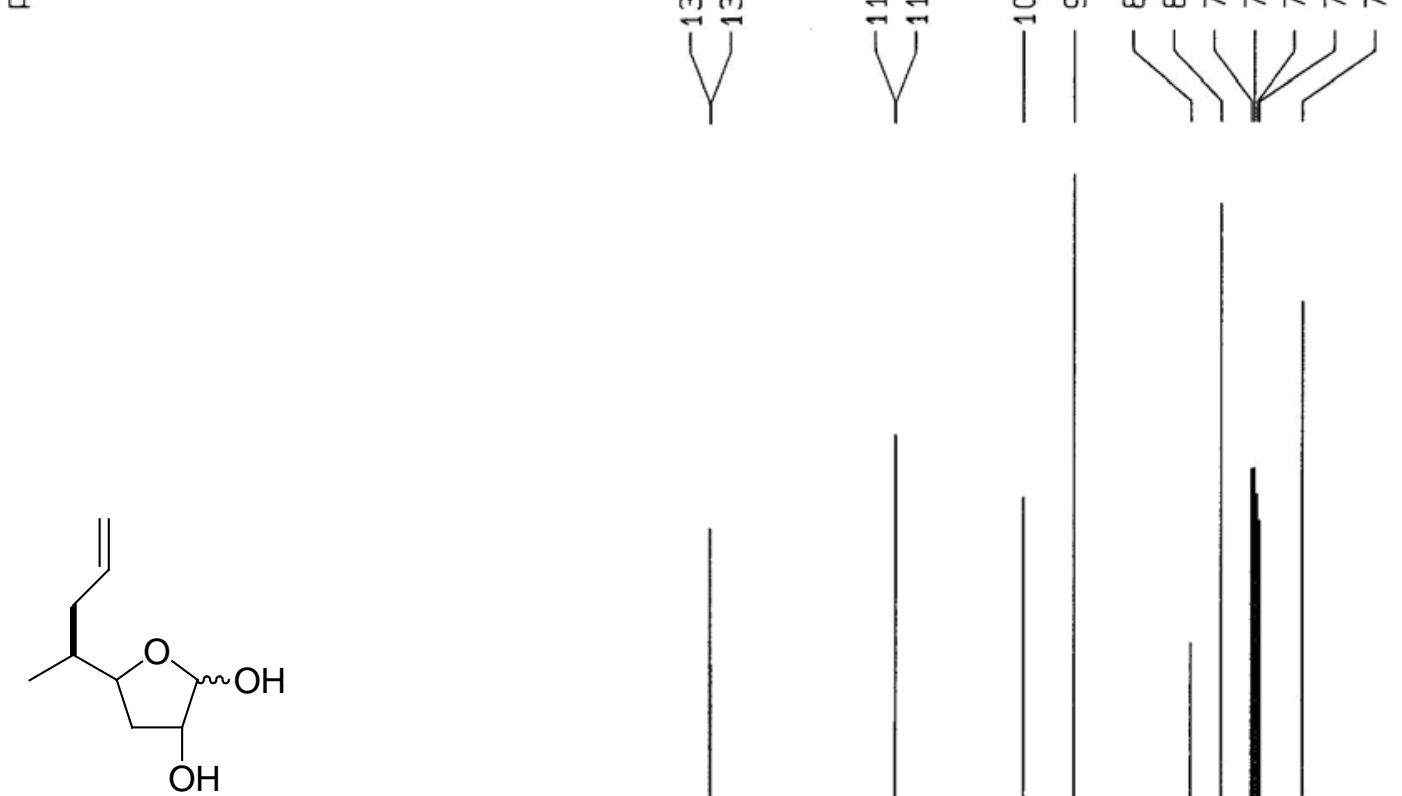

11

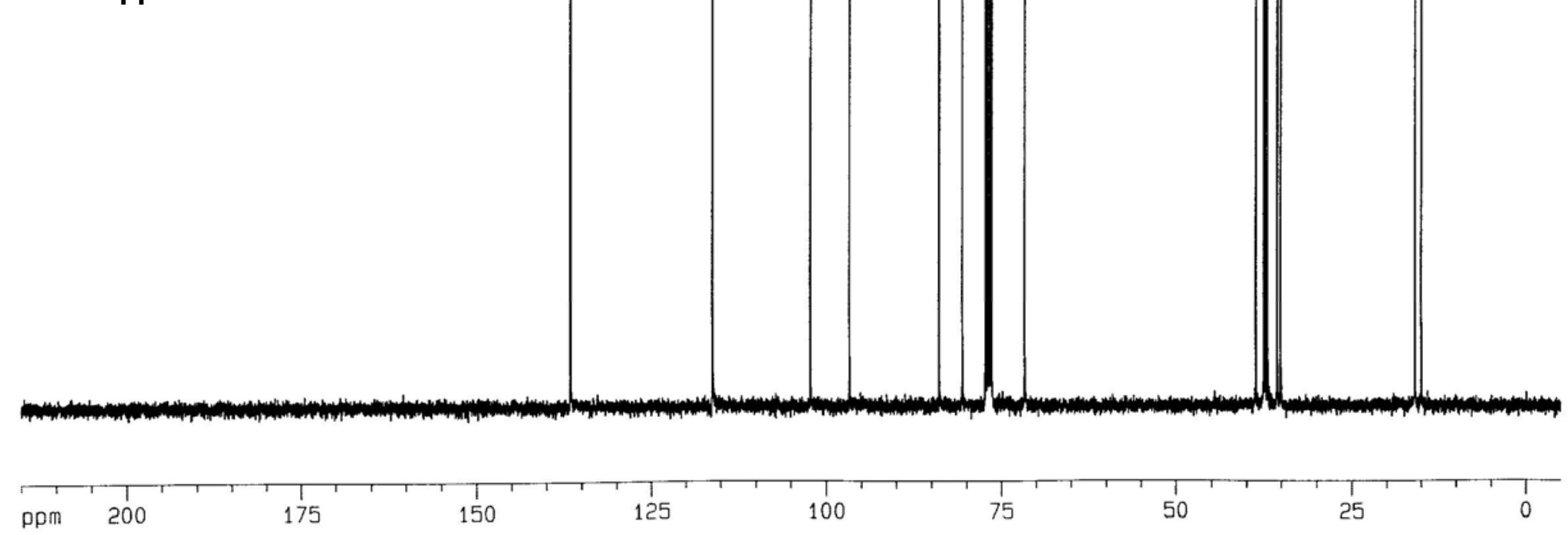




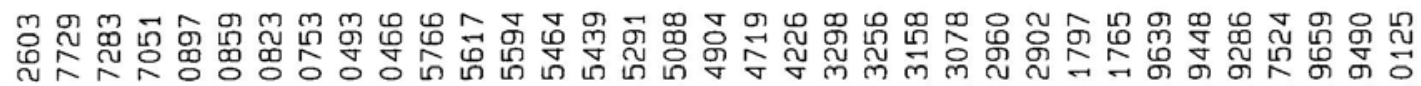

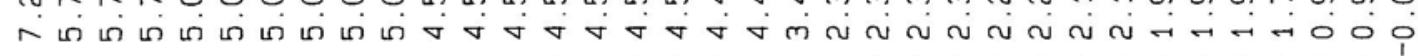
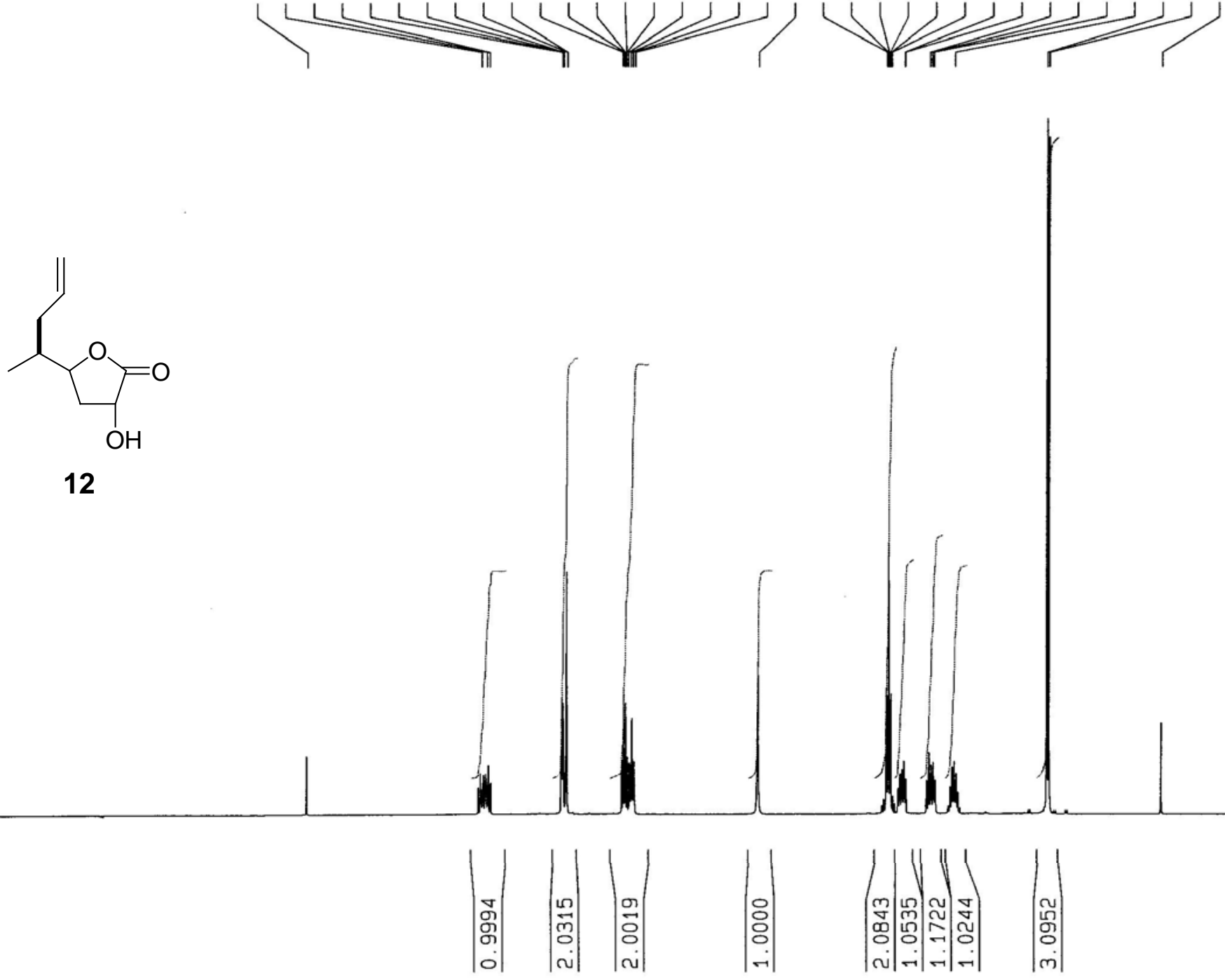


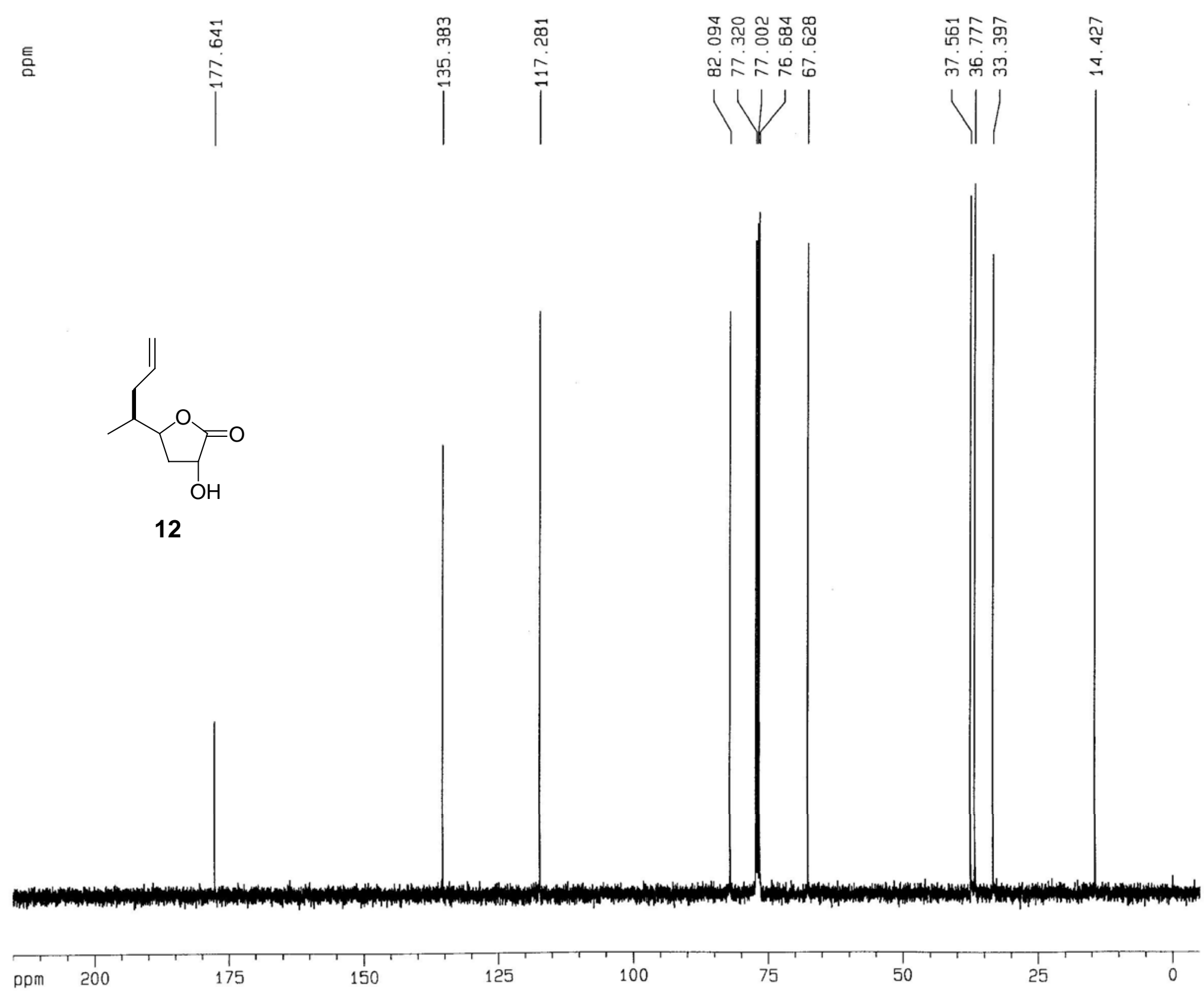




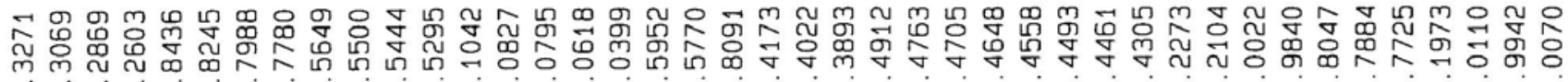
A N
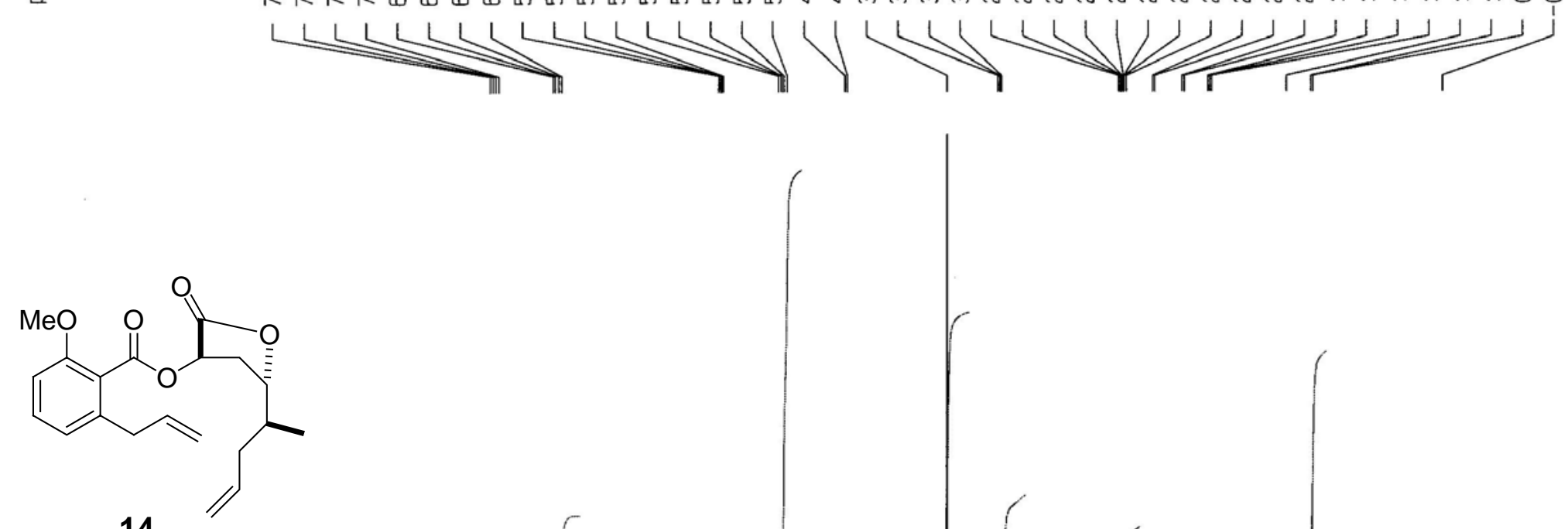

14

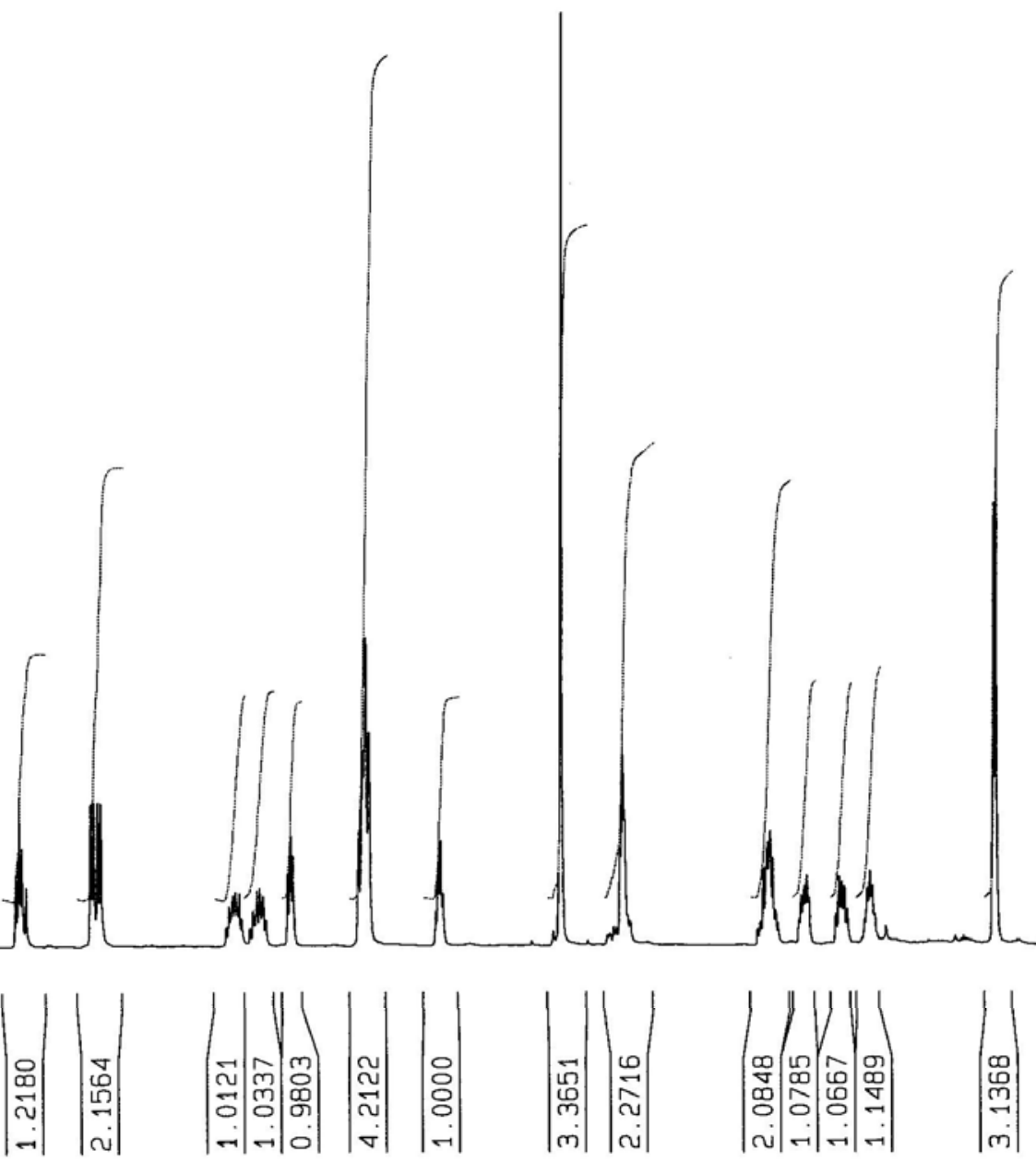




E
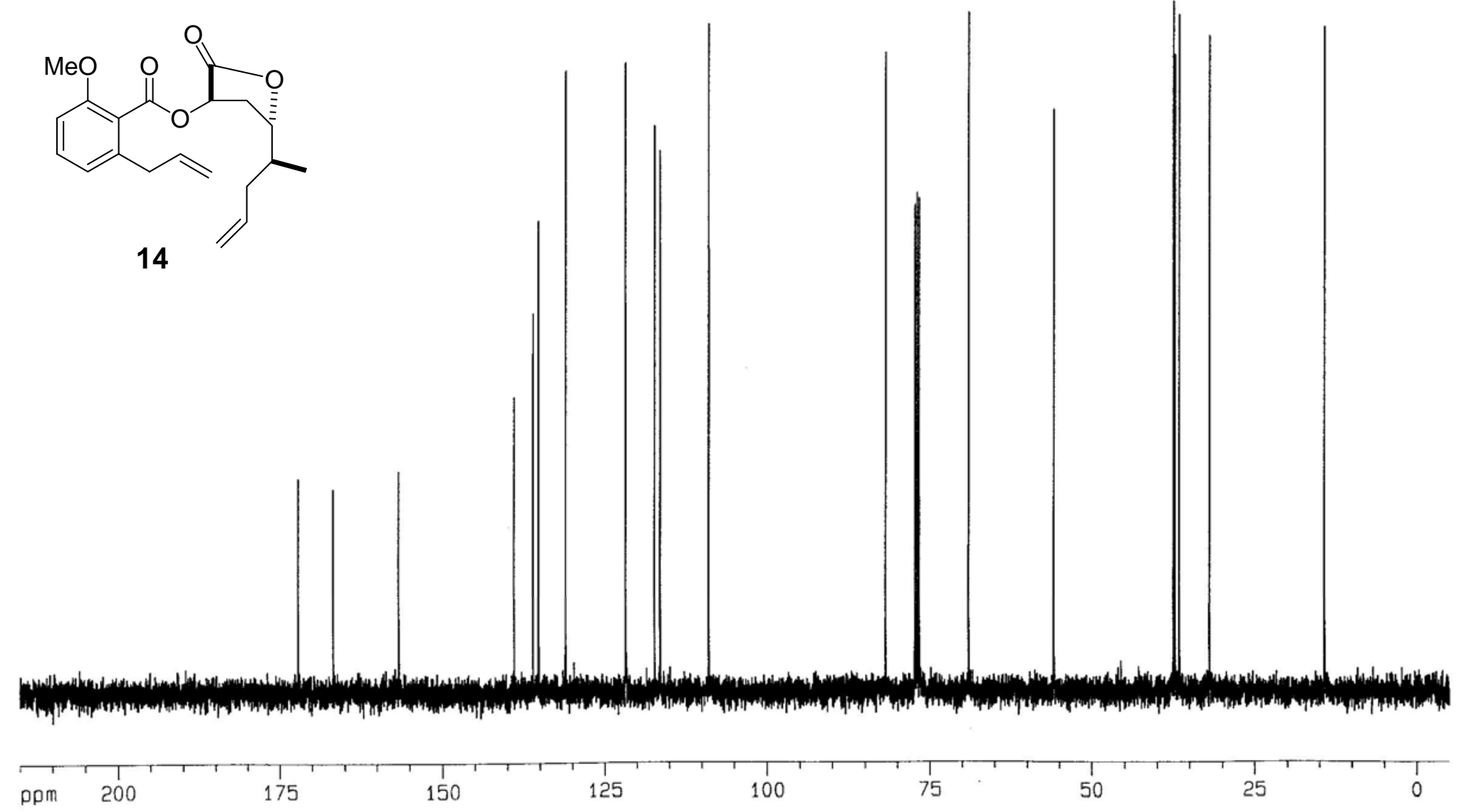


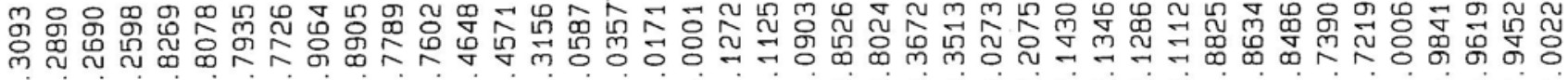
ヘ N ヘ ம
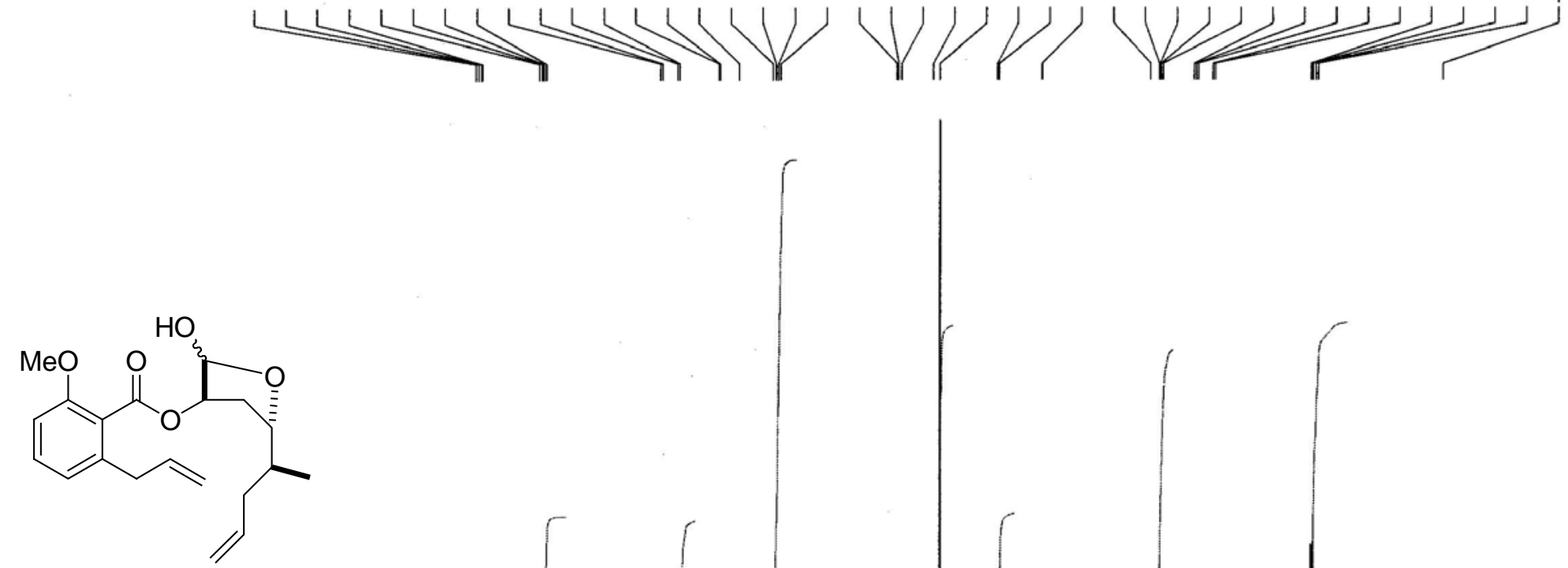

15

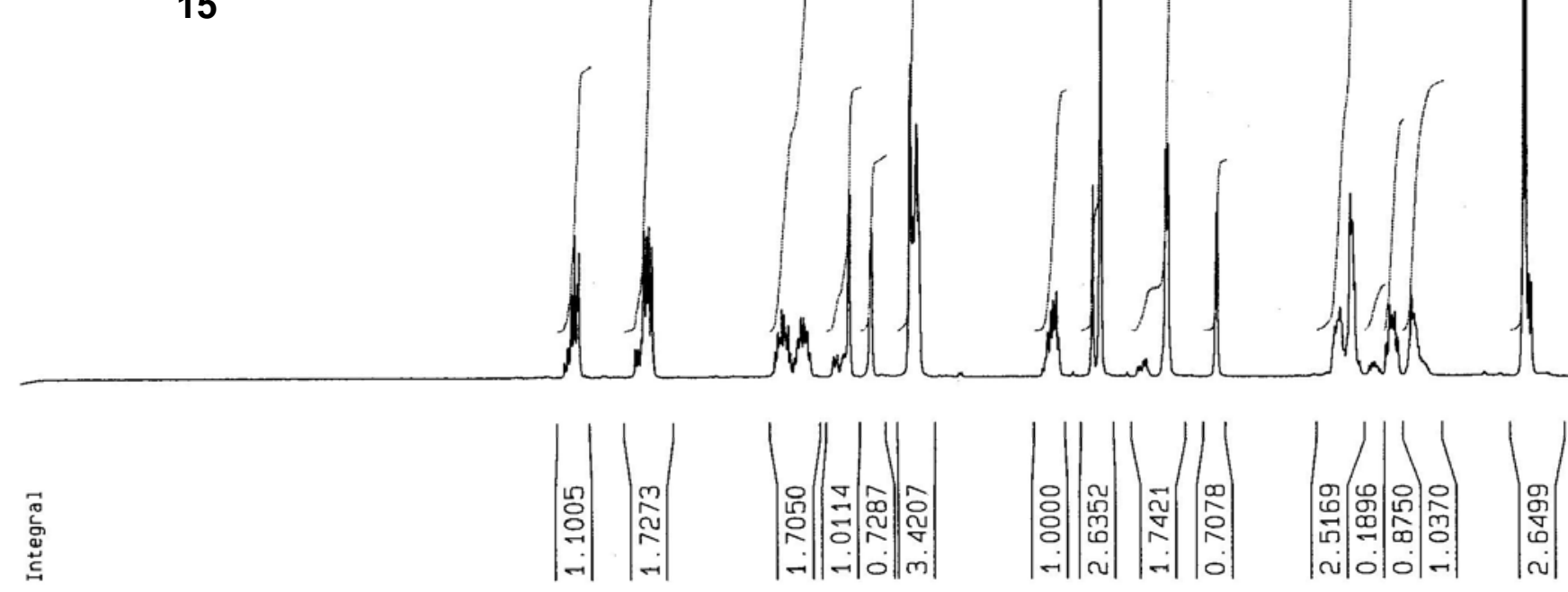




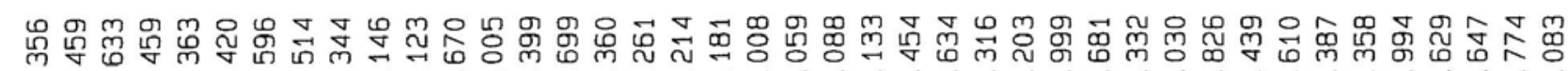

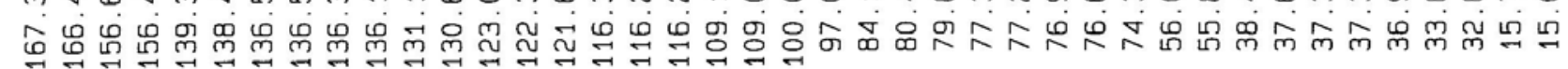
$\underbrace{0.15}$

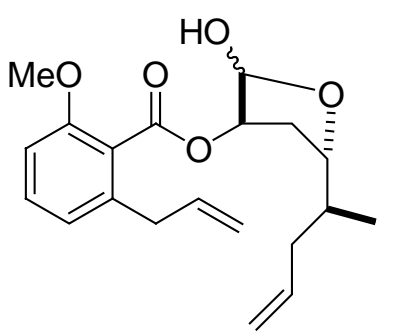

15

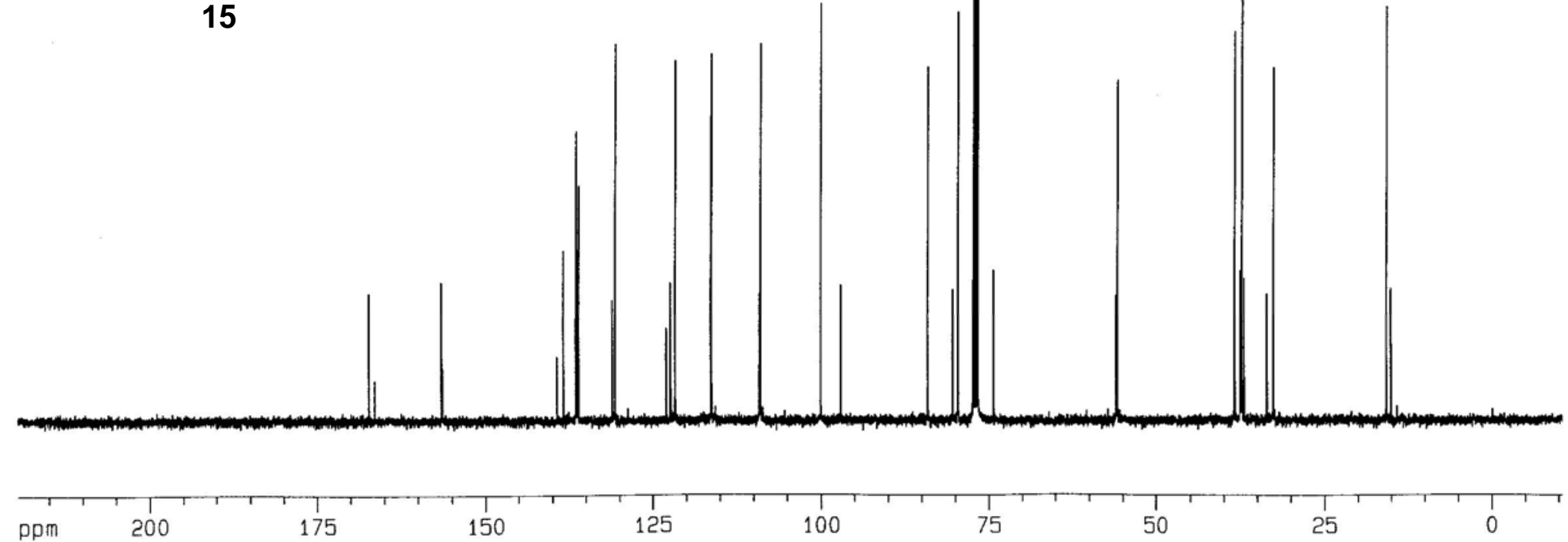




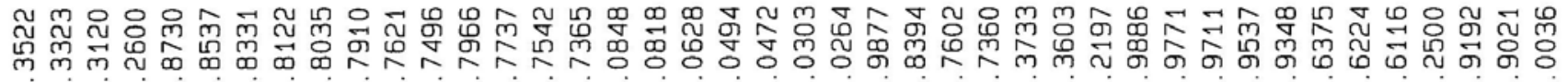

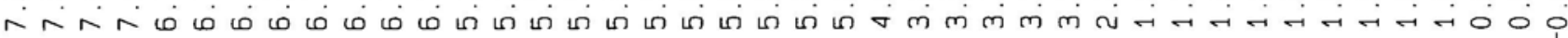
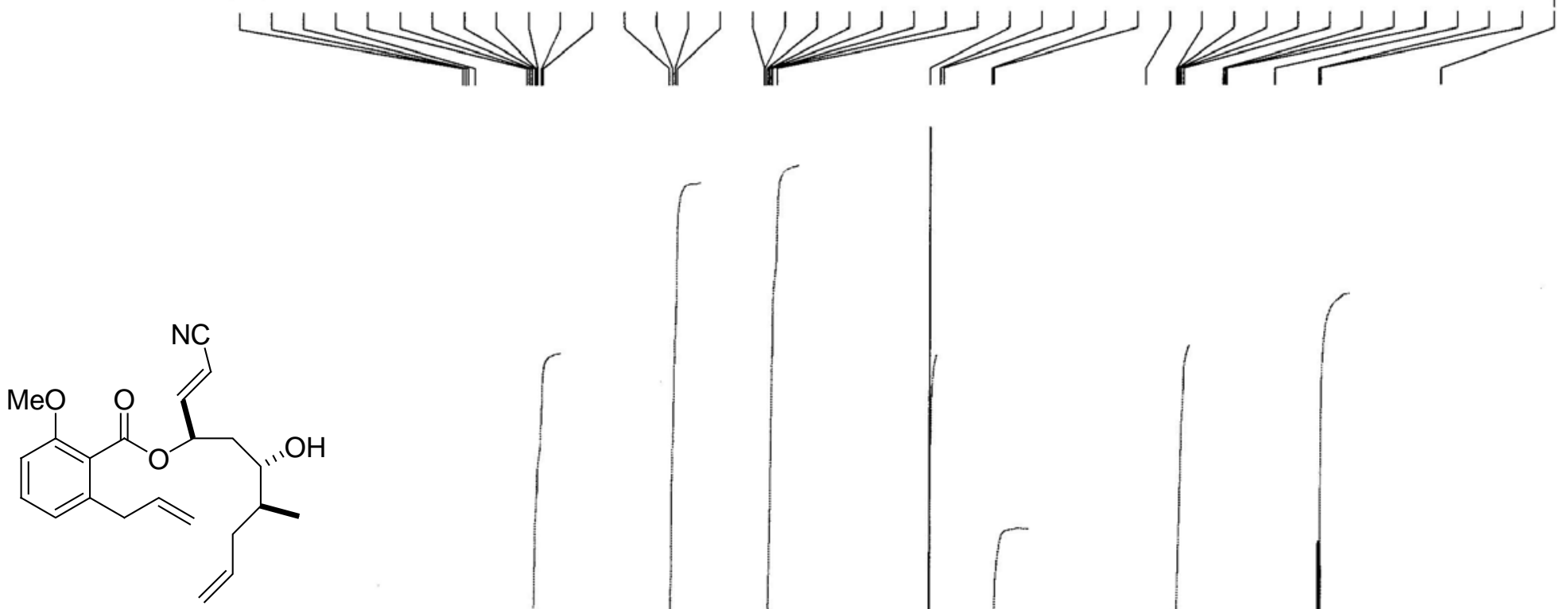

16

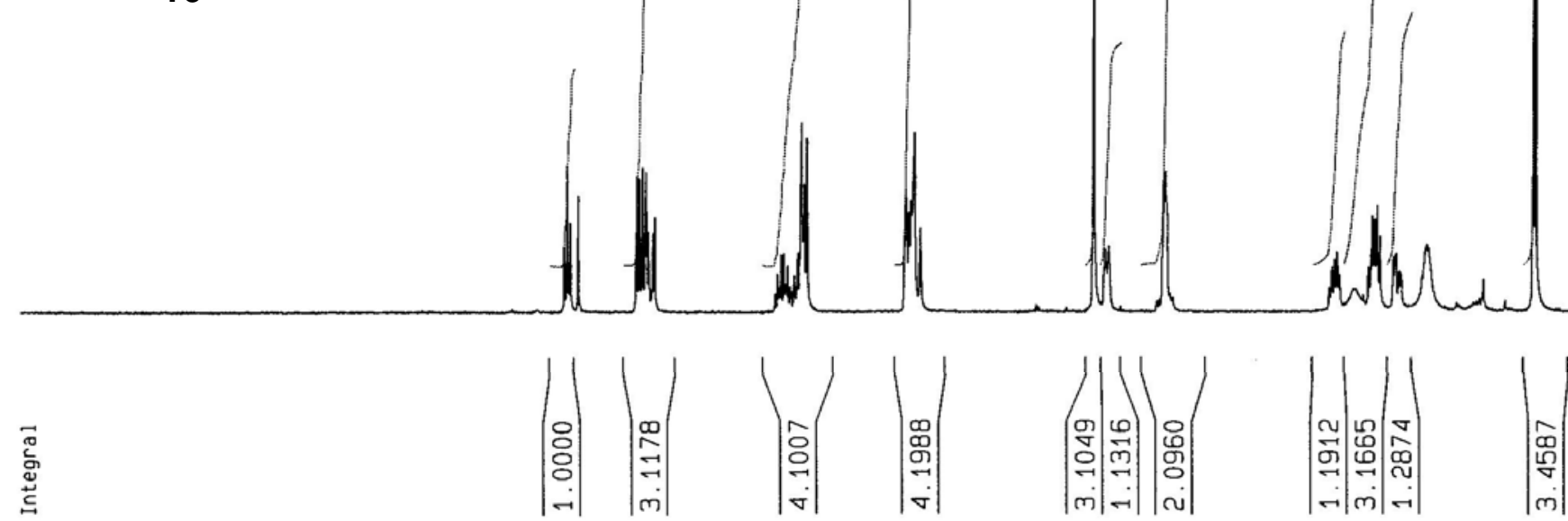




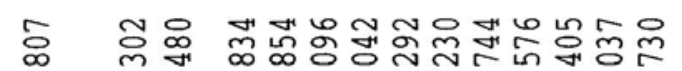

迆

엉요요

क 구윙

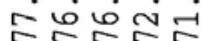

望

inimin

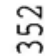

$\vec{\imath}|\overrightarrow{\mid}|$

YI

4

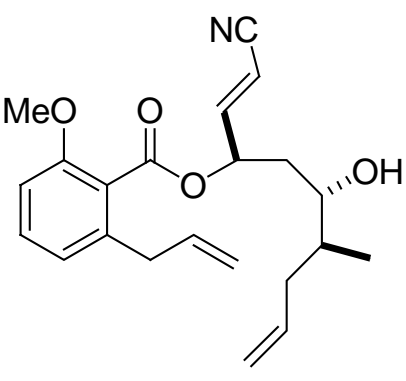

16

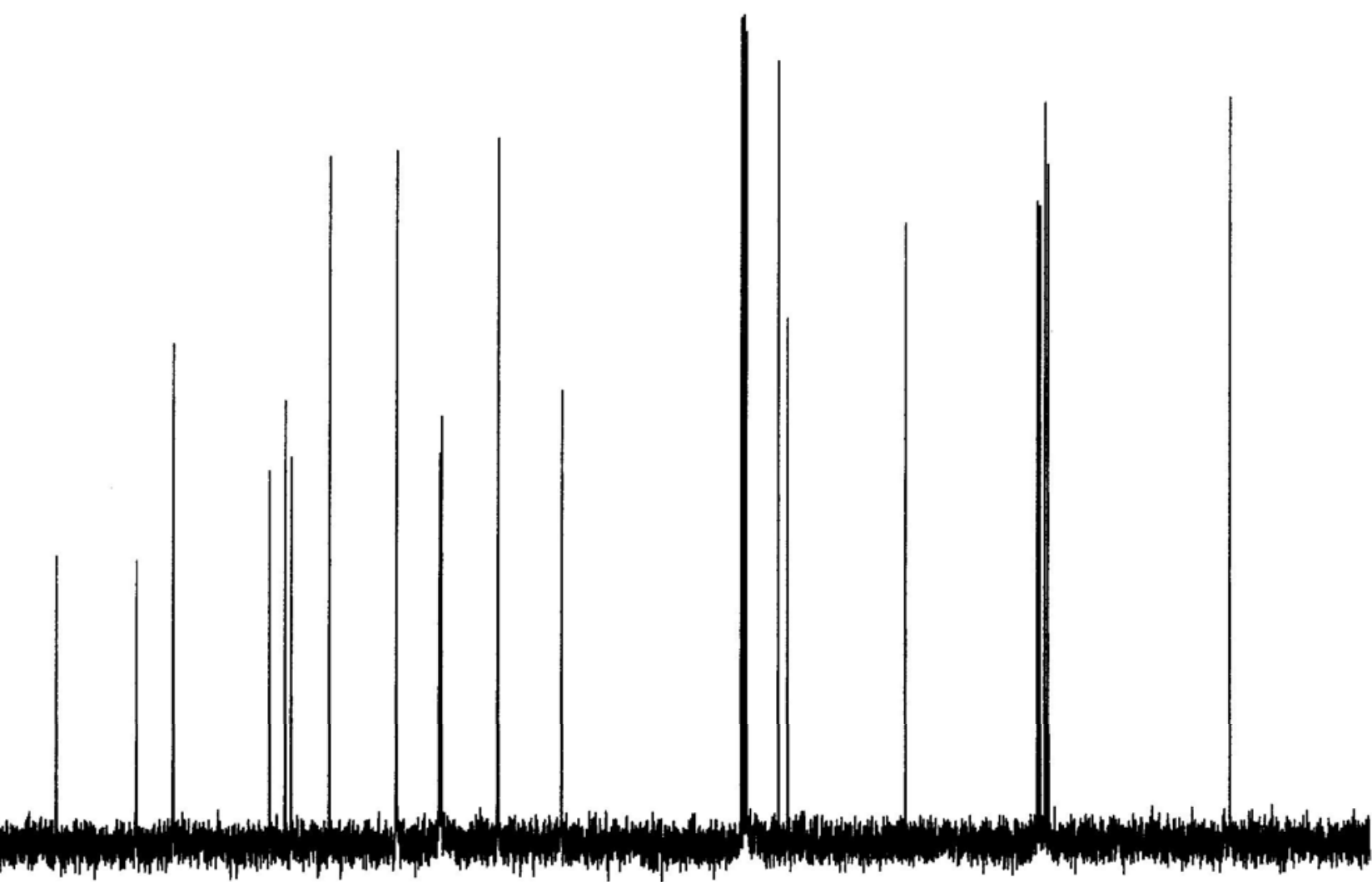




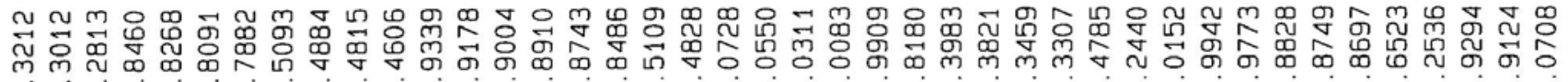
Nn ம
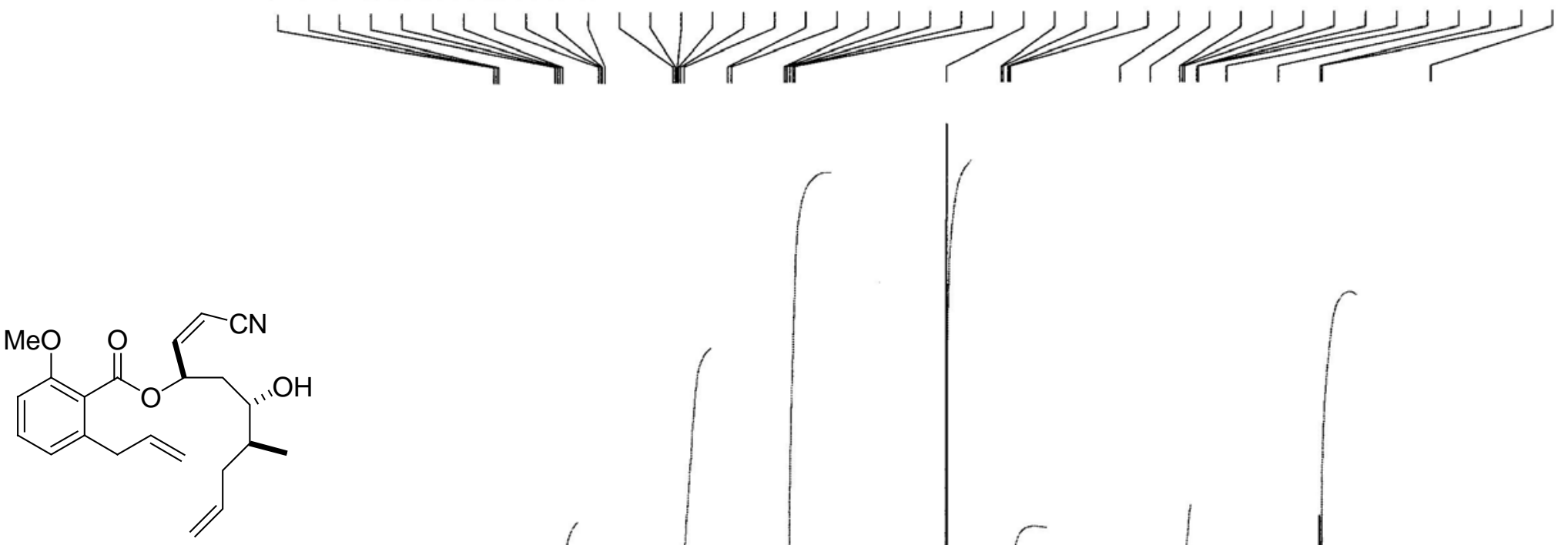

17
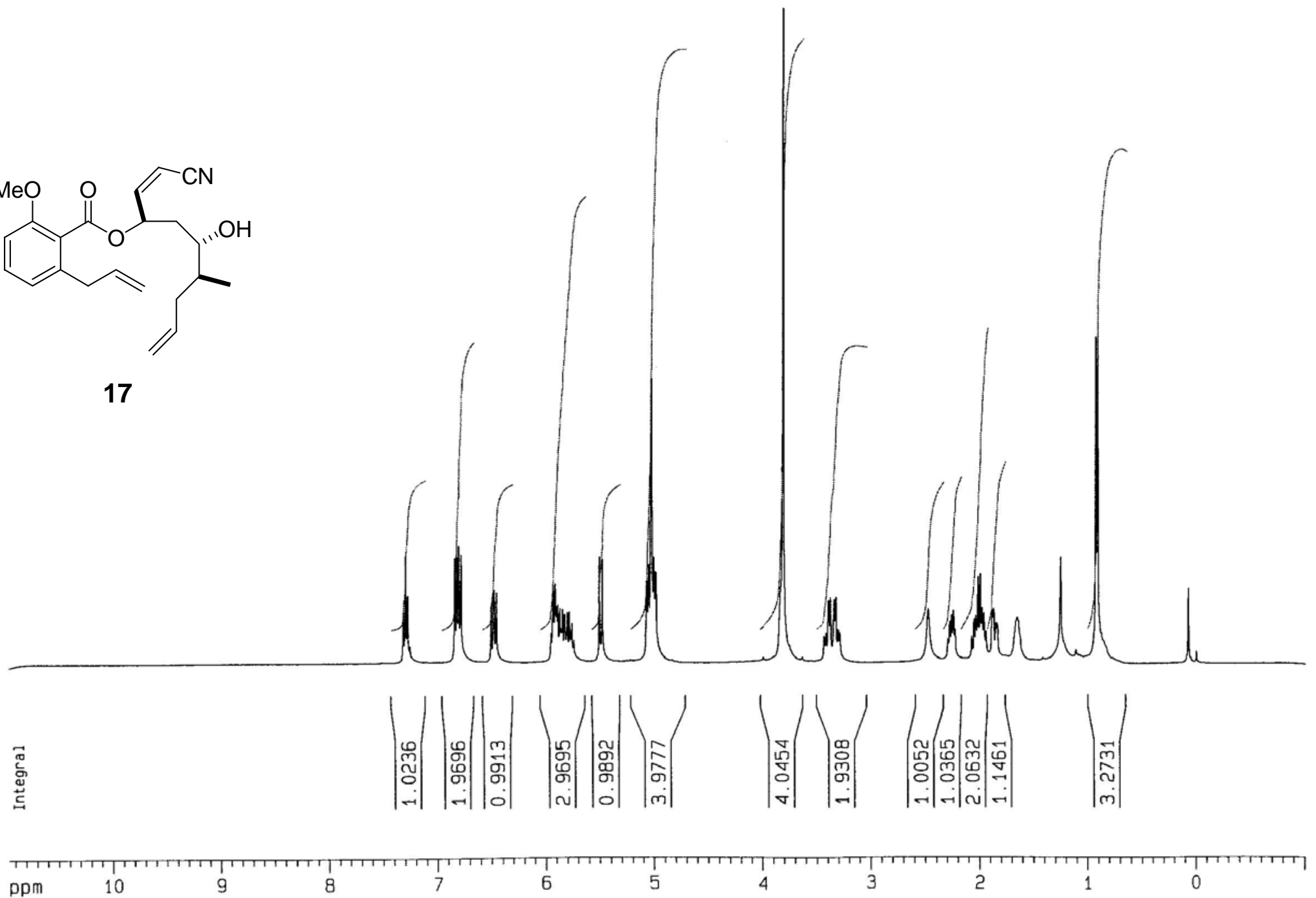


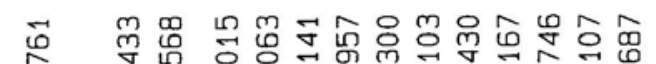

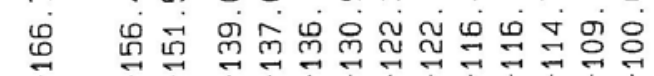

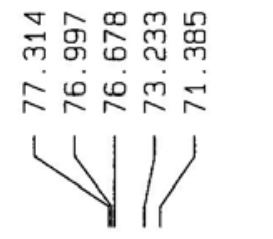

$\underset{\infty}{\infty}$

荧离命命界

111

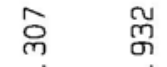

$\stackrel{m}{\rightarrow} 0$

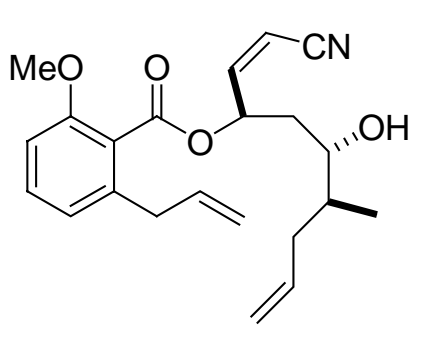

17

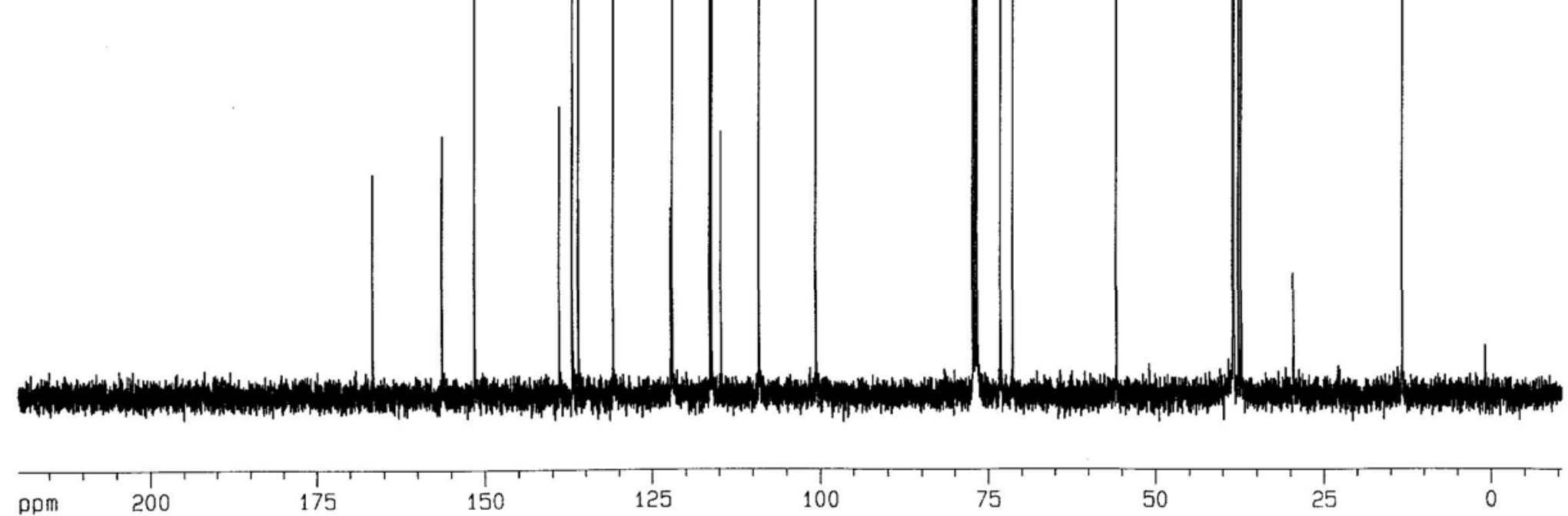




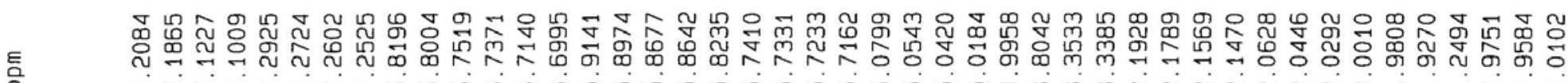

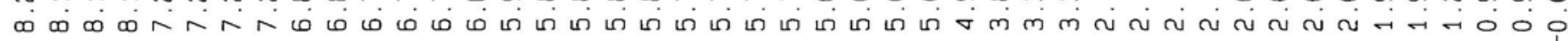
$\underbrace{2}_{1}$

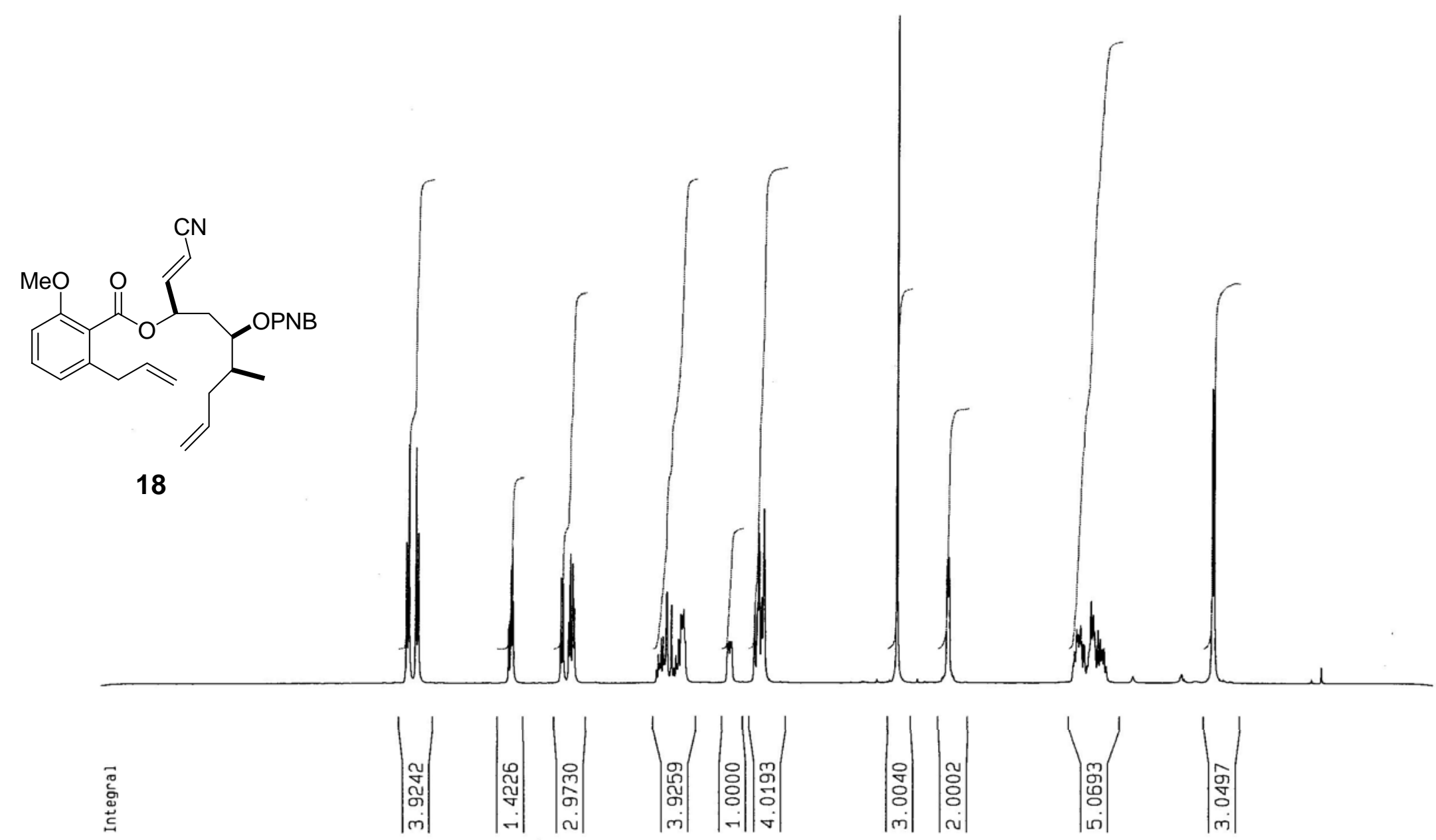




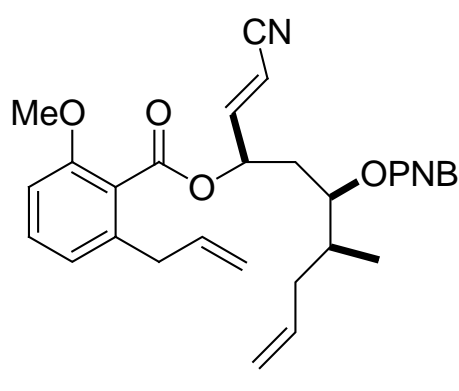

18

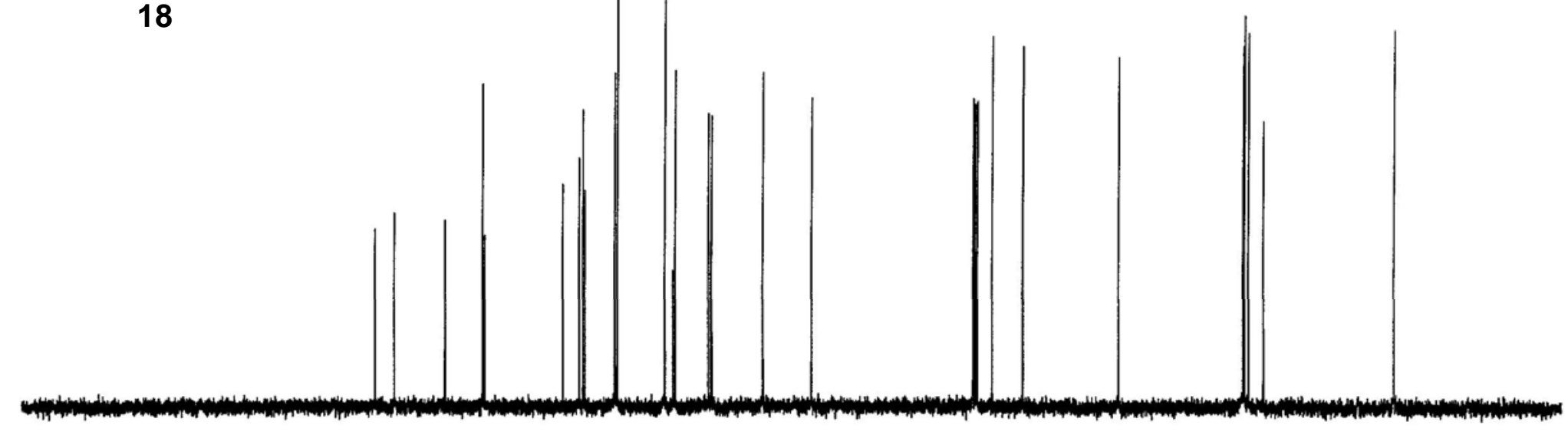




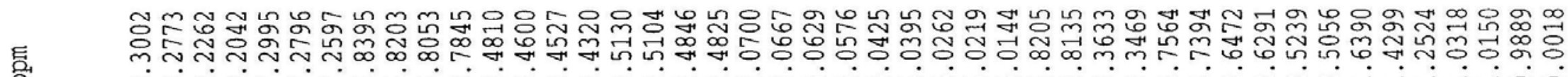
o $L_{\pi}^{L L}=1$

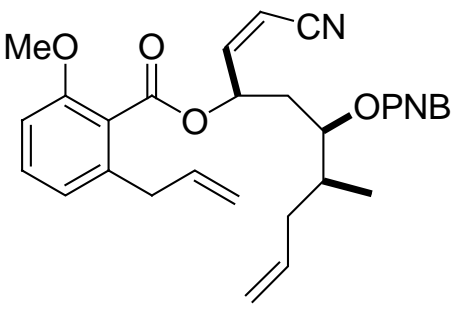

19 


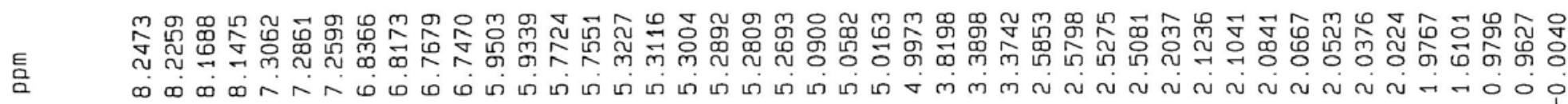
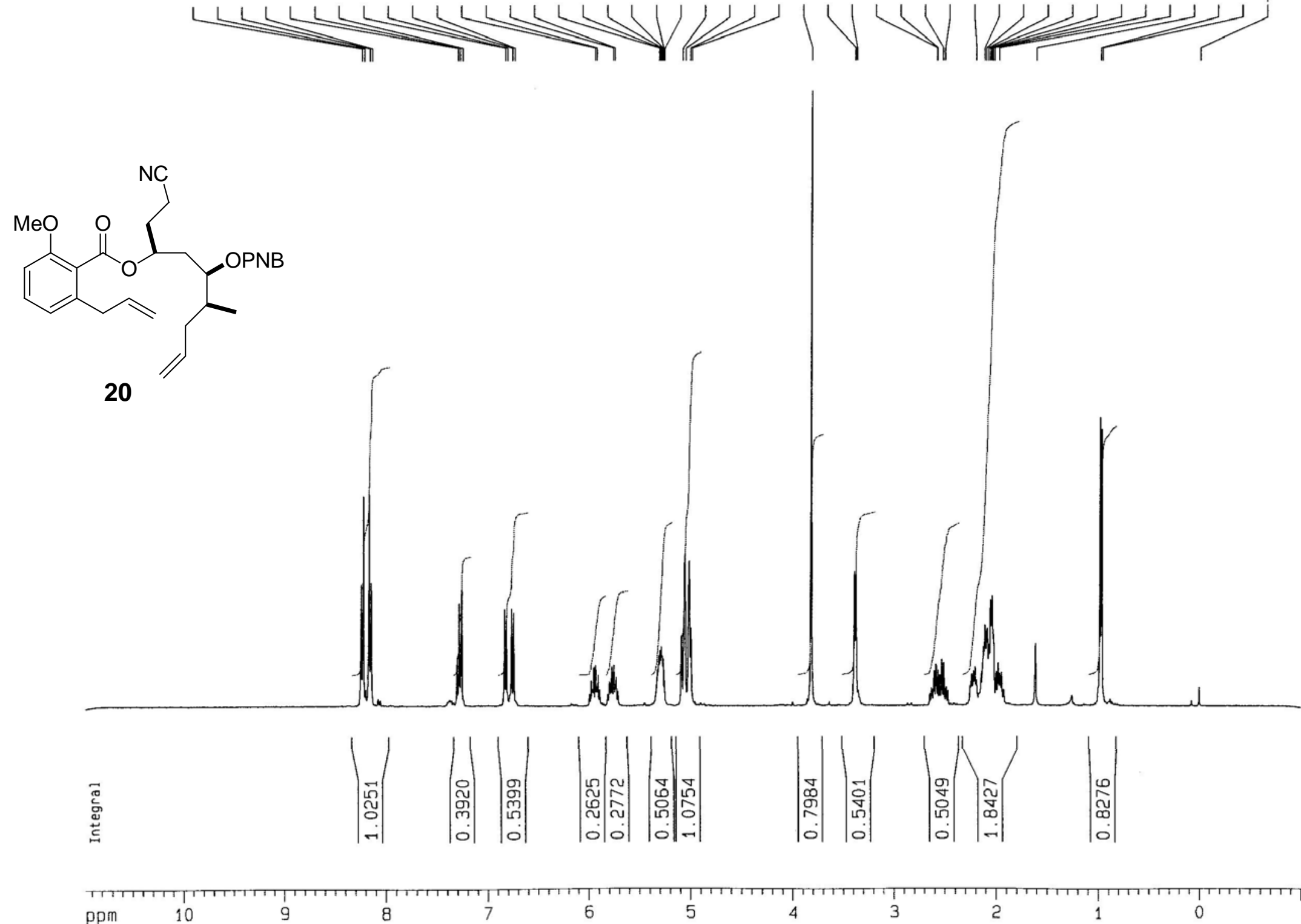


$$
\text { v }
$$


镸

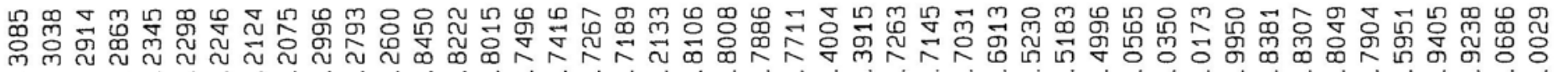

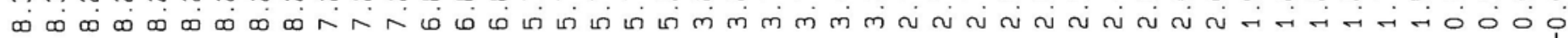
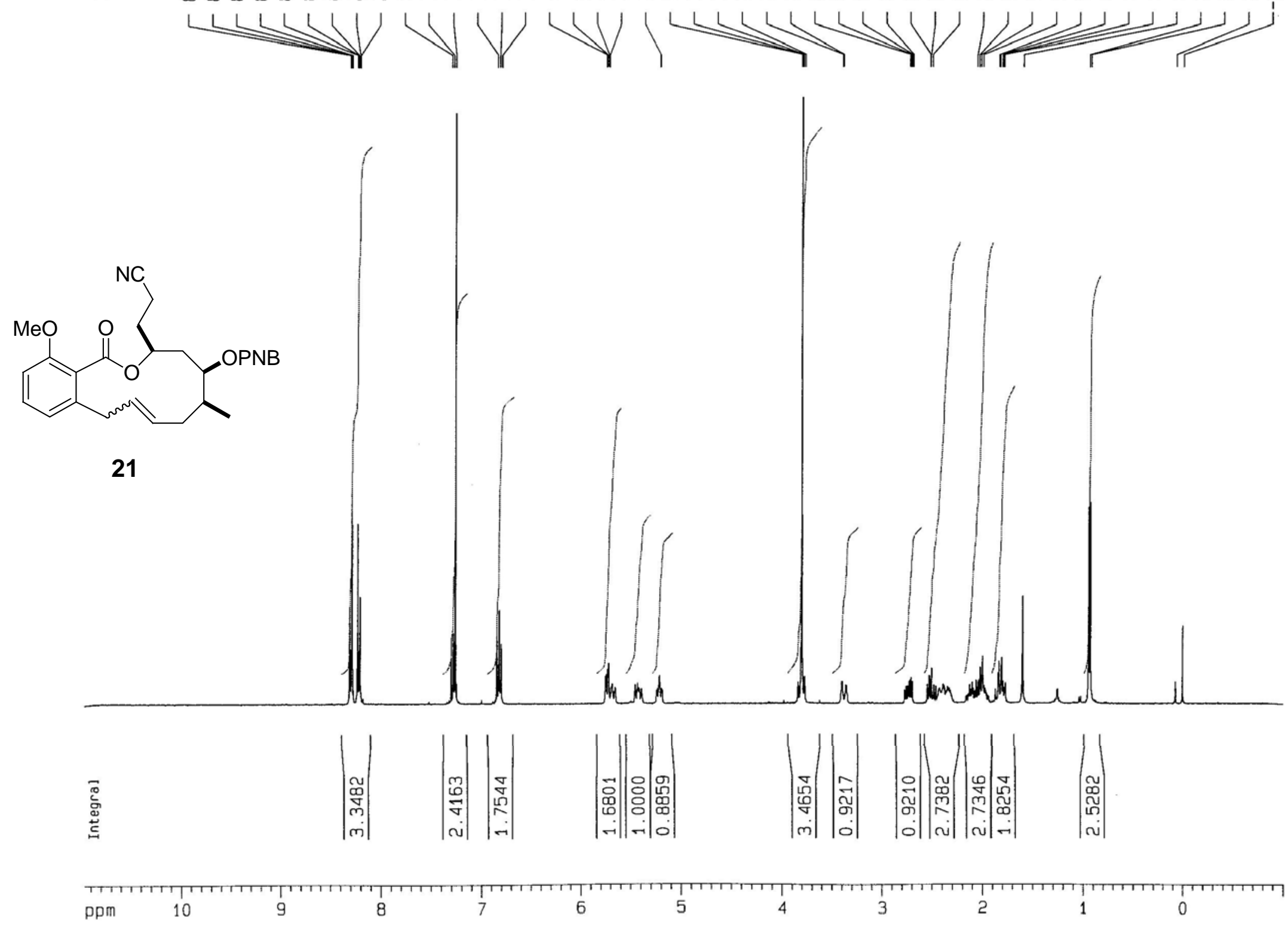


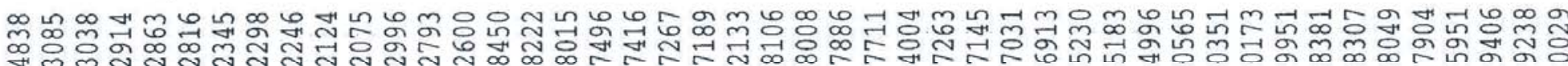

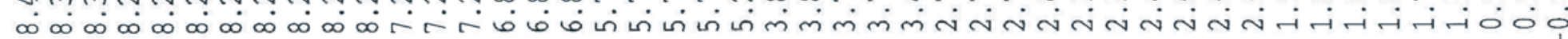
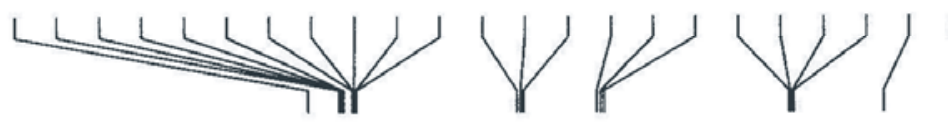

HPLC results (Silica gel 60):

Gradient ethyl acetate in hexanes

$0 \%$ ethyl acetate to $30 \%$ ethyl acetate

(30 minutes)

Peak 1 (23.6 min): 5.3\%

Peak 2 (29.0 min): $94.7 \%$

NMR integration results:

$E: Z 18: 1$

Ratio determined based on NMR and HPLC
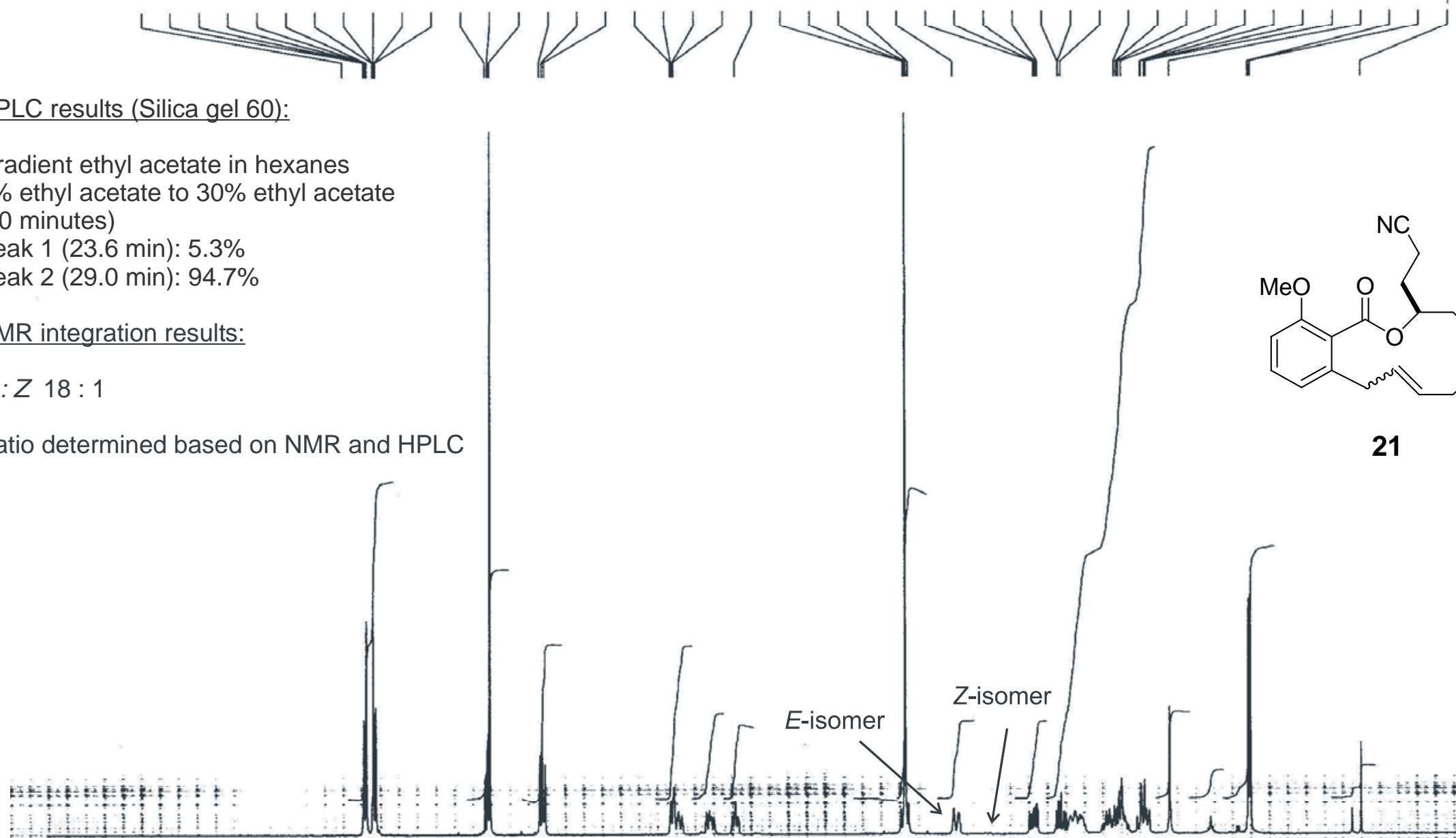

$1+11$

M.
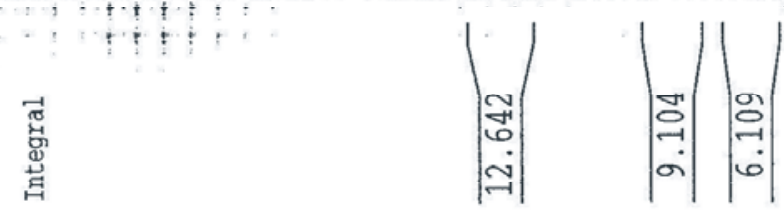

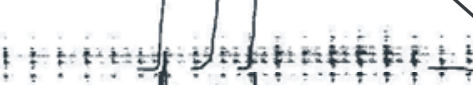

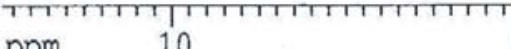

8
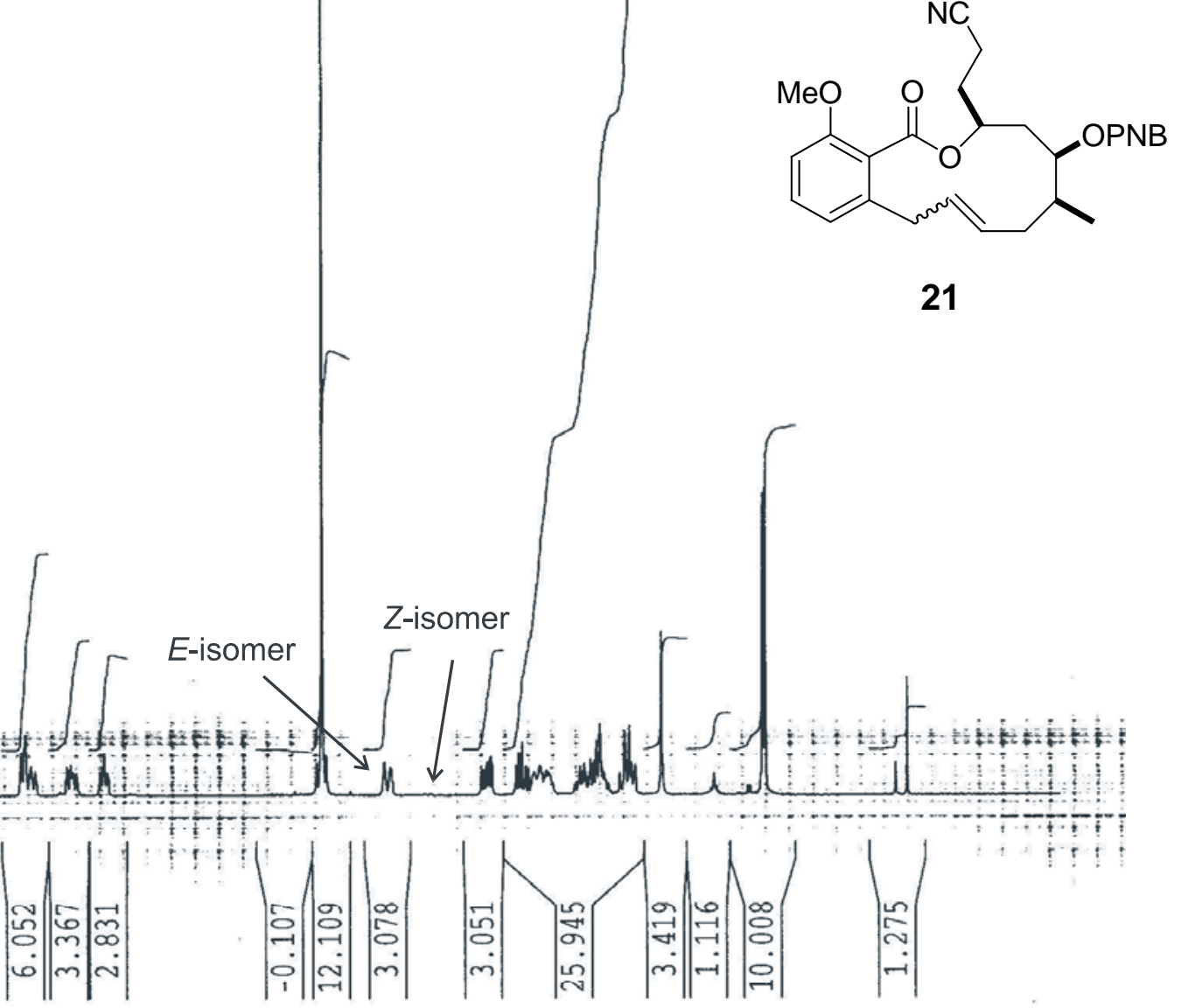

21 


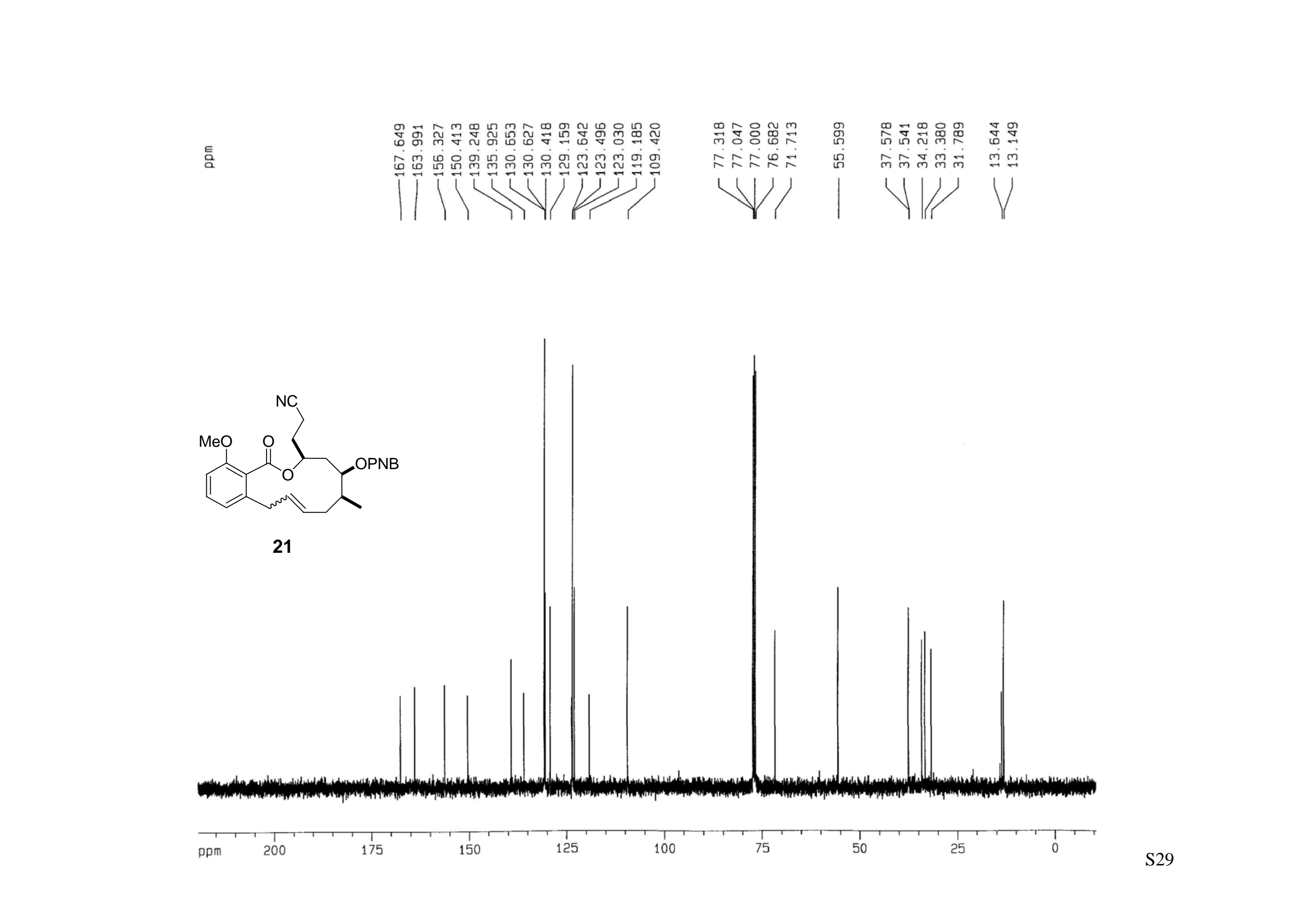




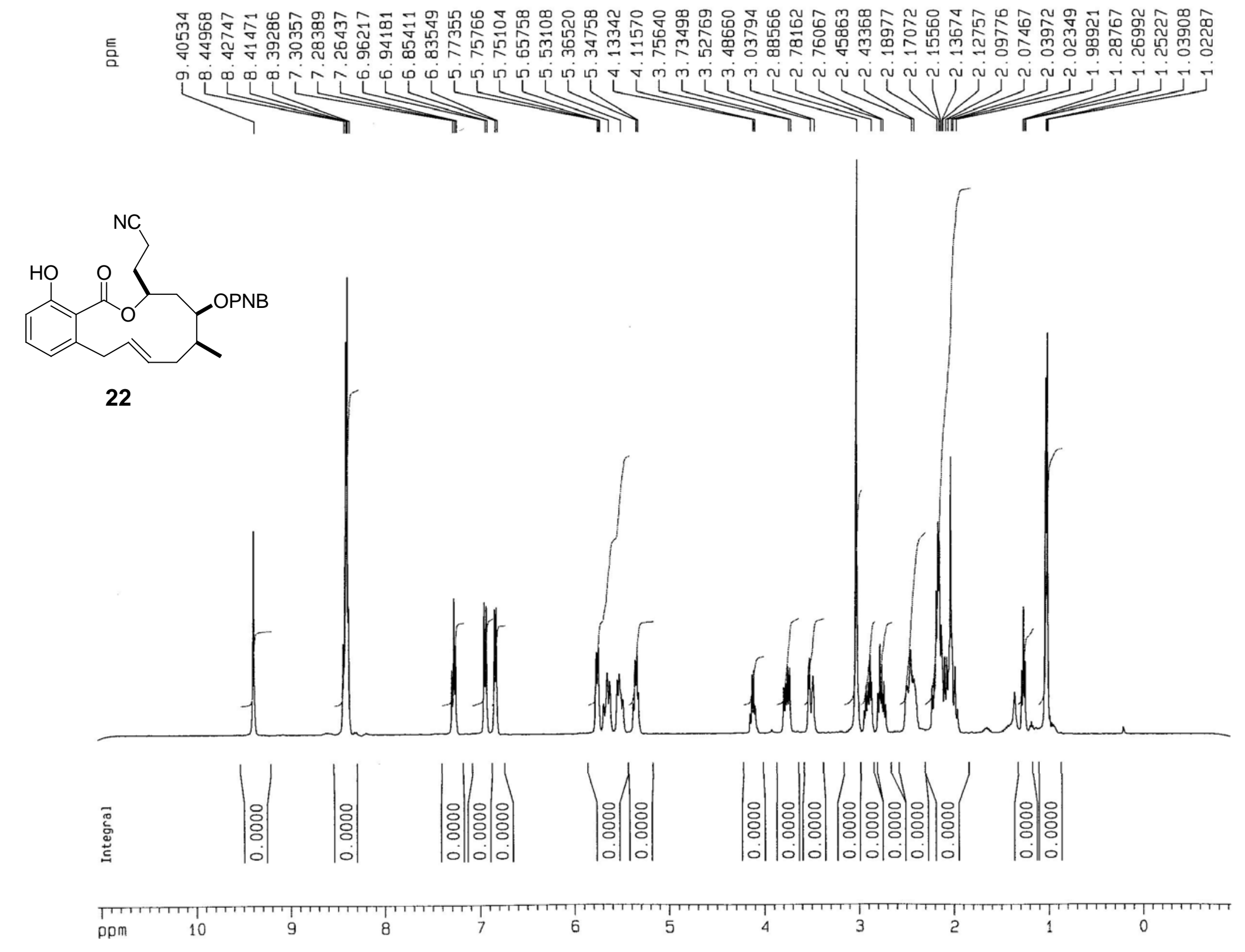




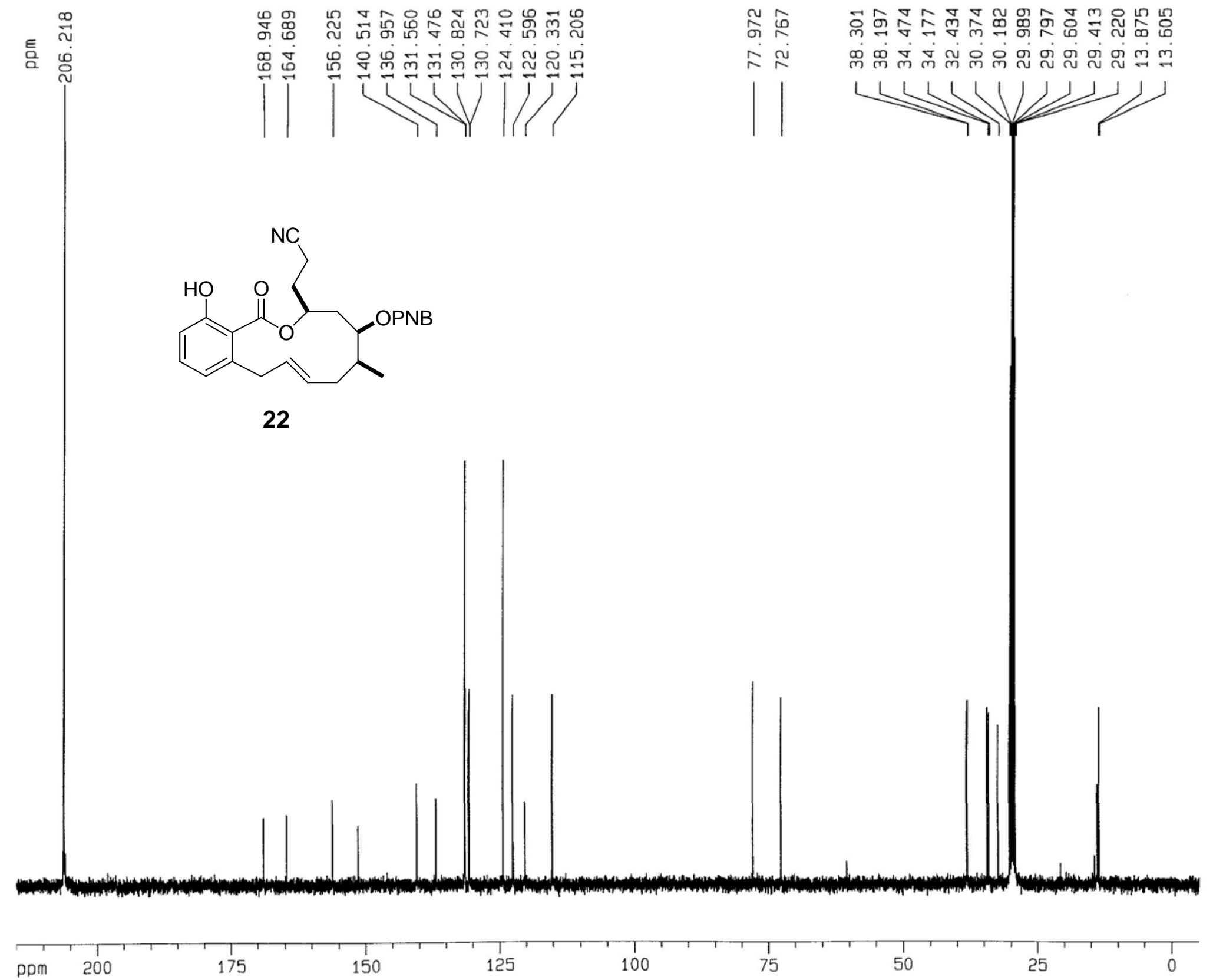



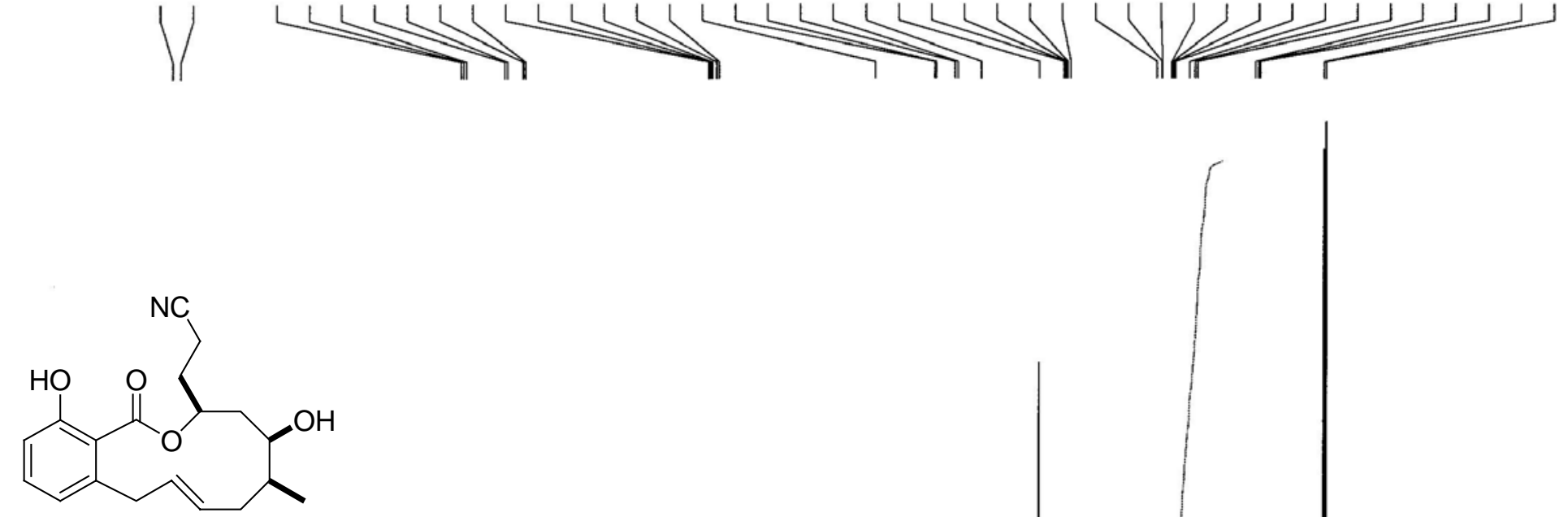

23

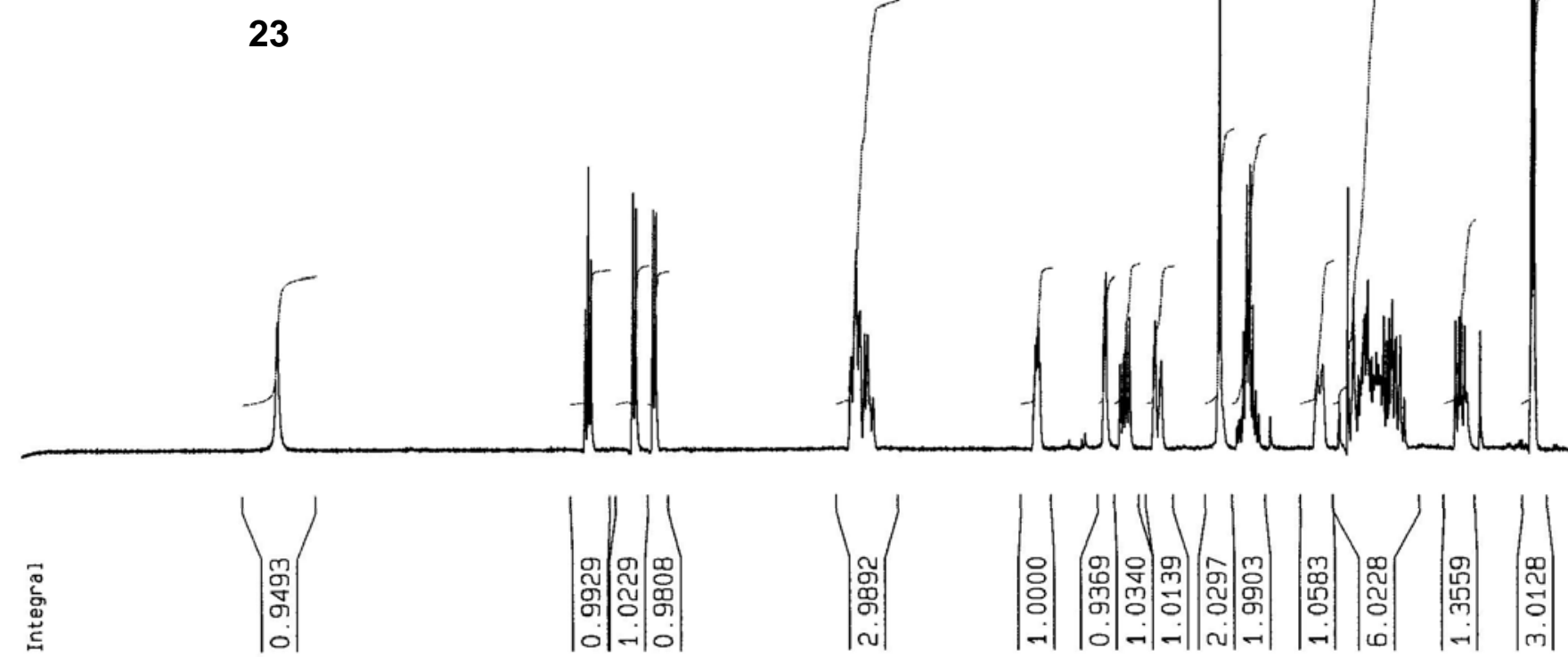




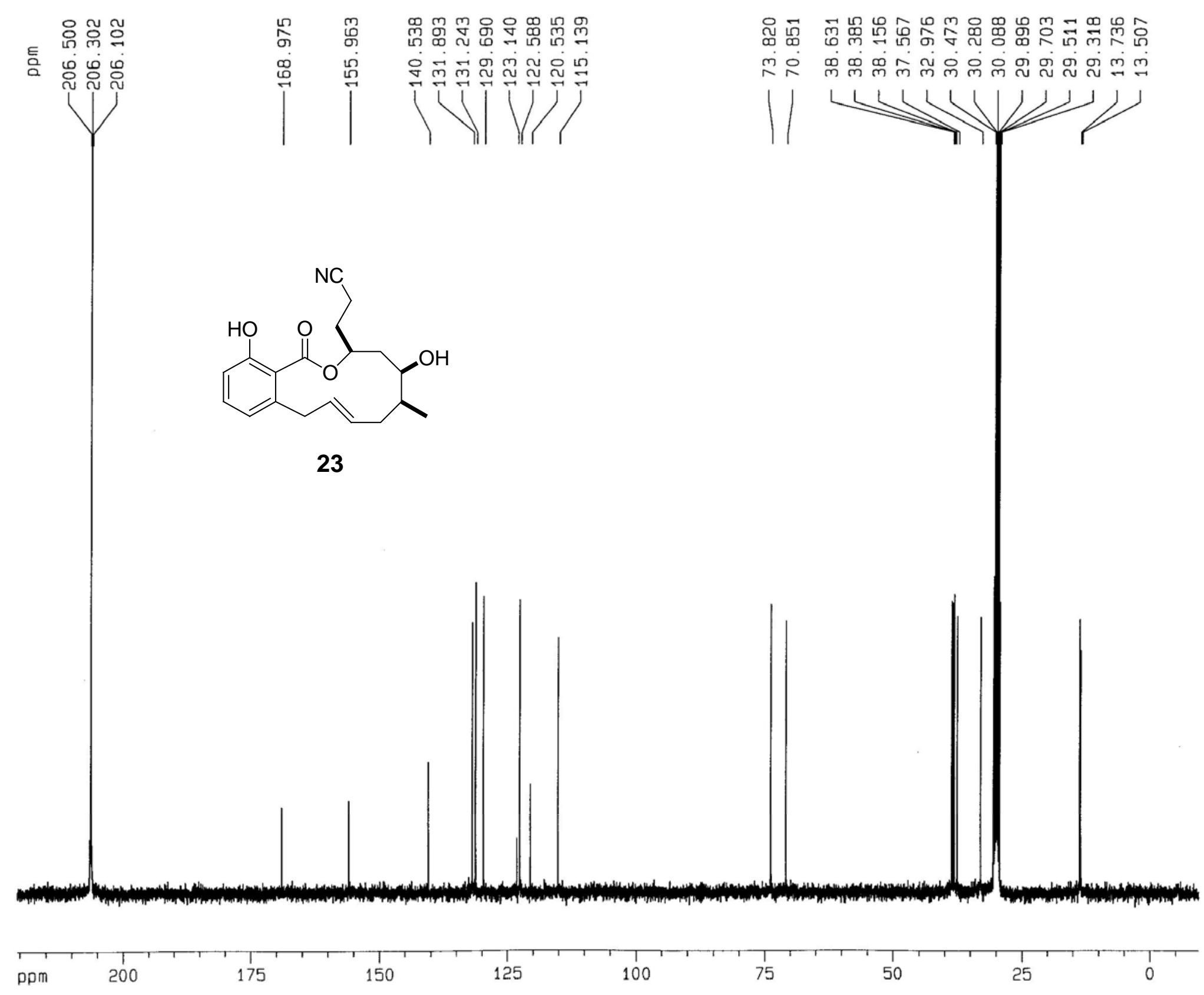




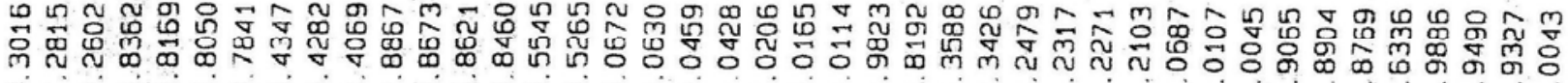
- N
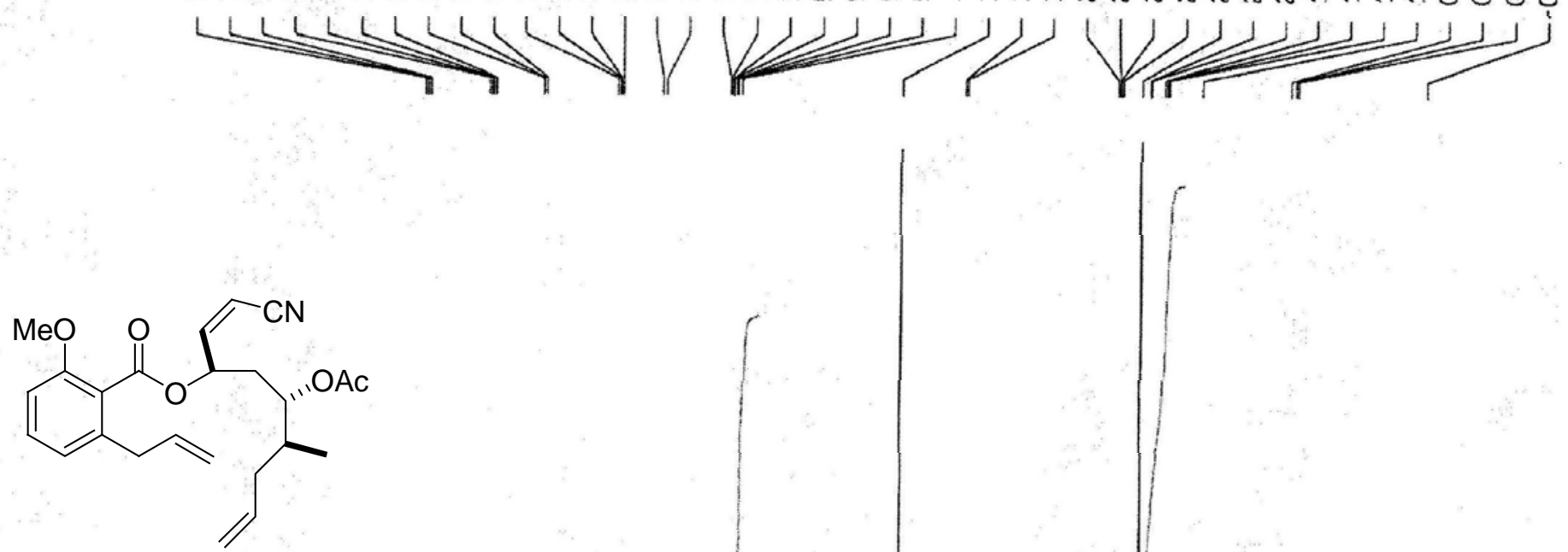

25
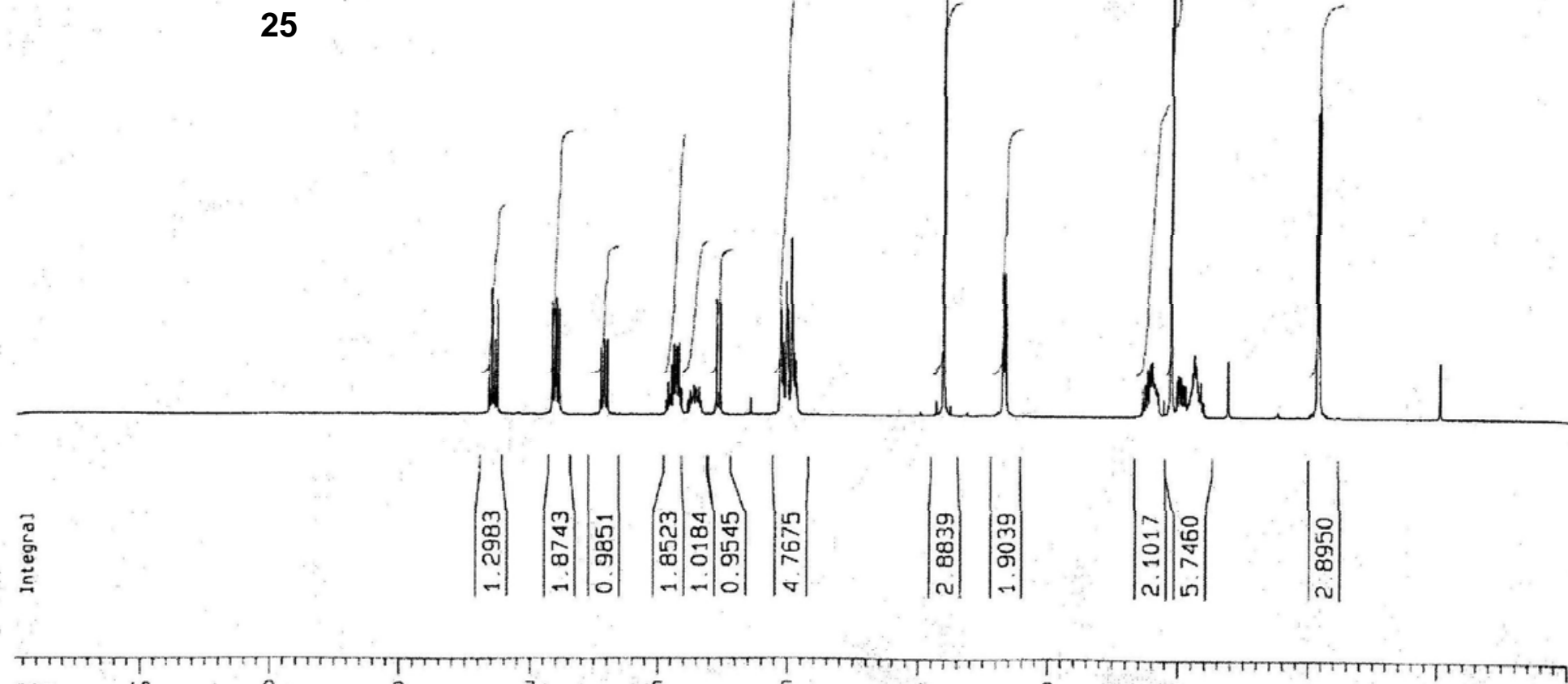


$$
4
$$




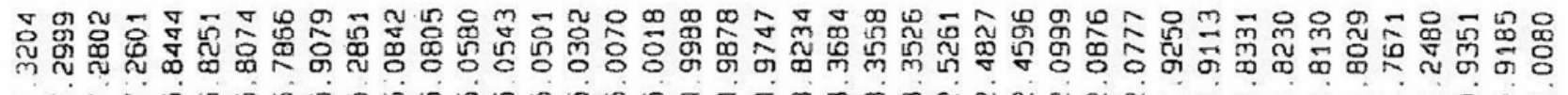
- N N

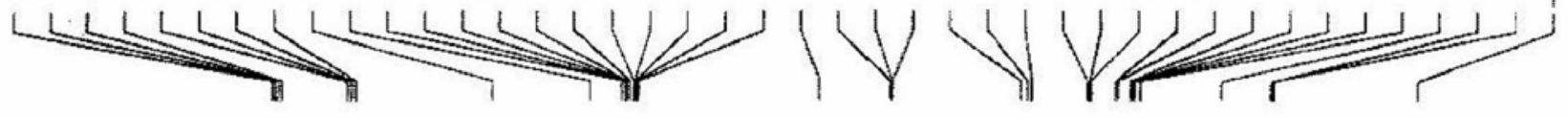

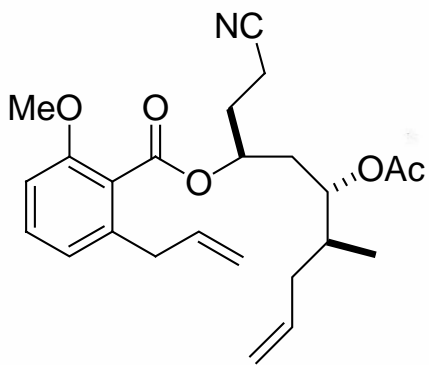

26

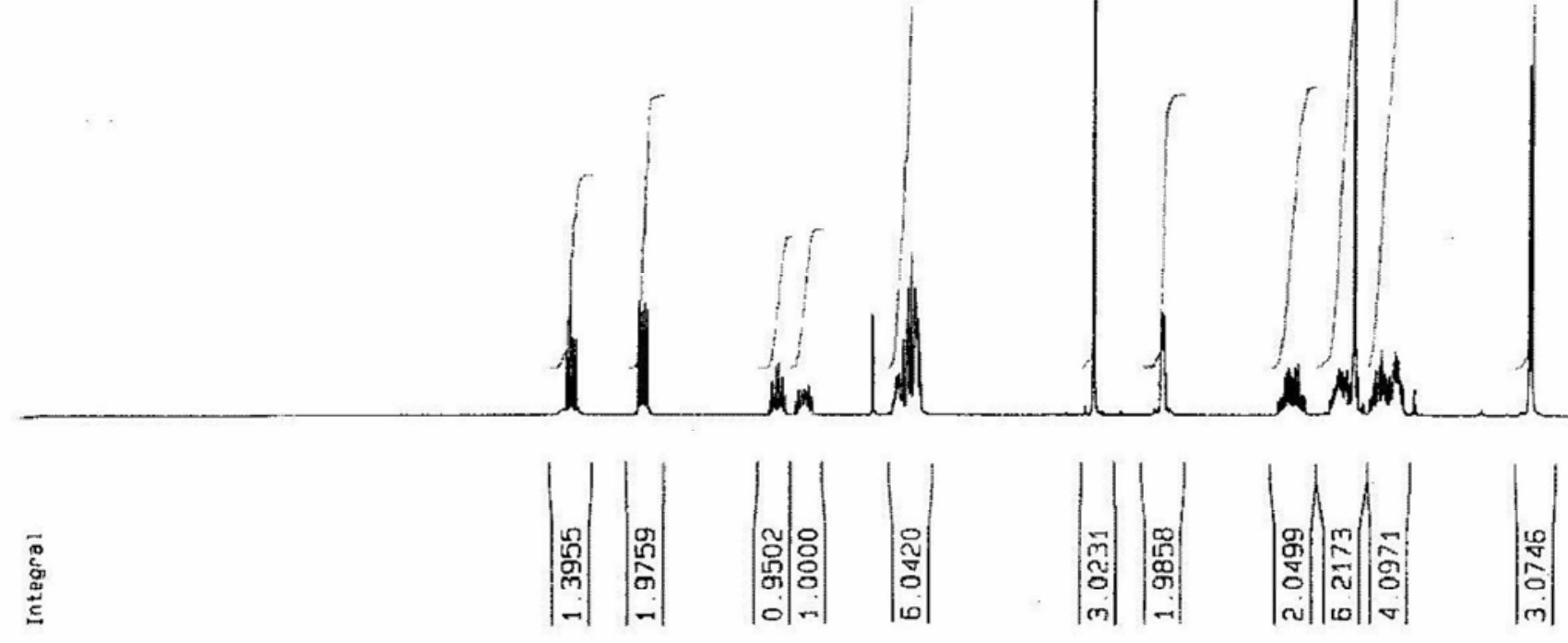




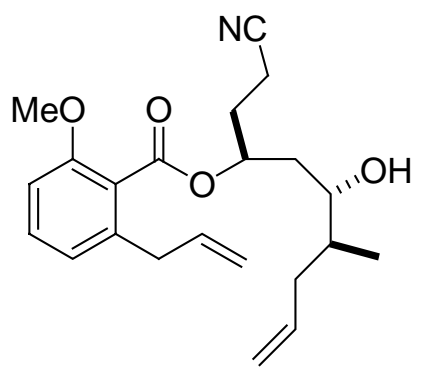

27

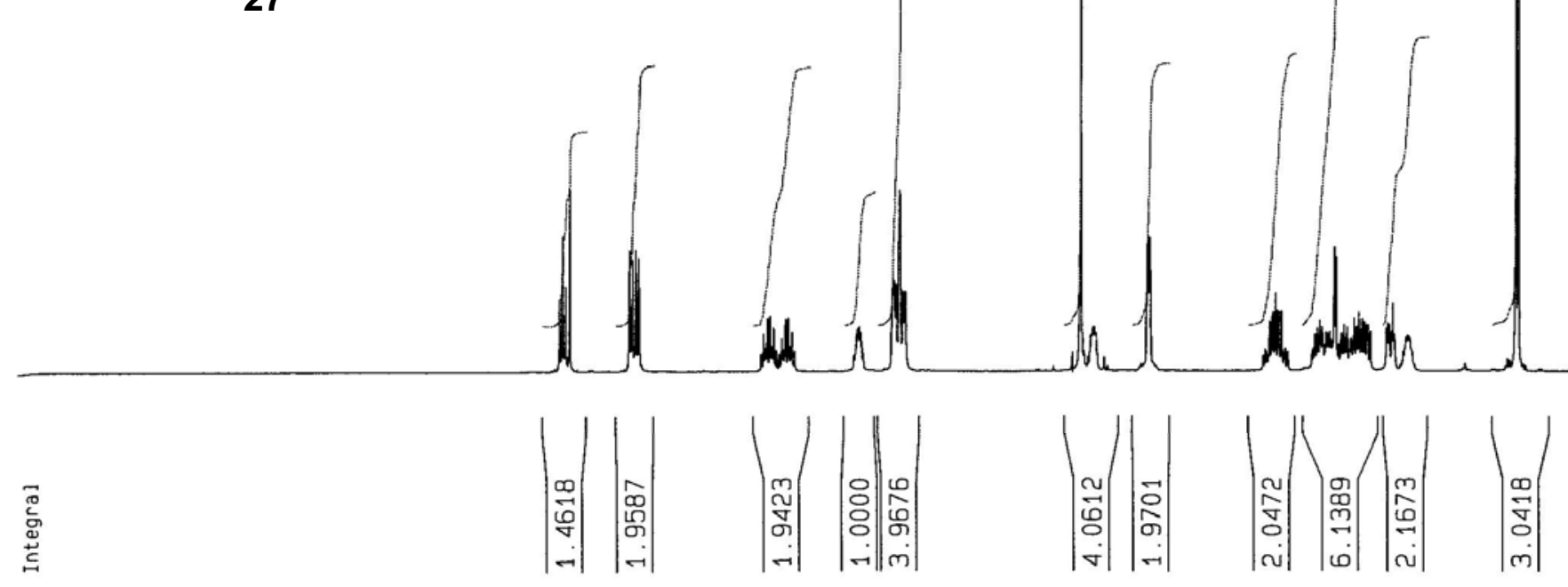




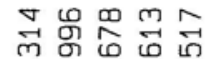

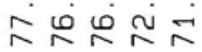

WI

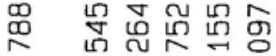

出

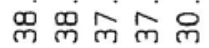

Y

兽

mim

V

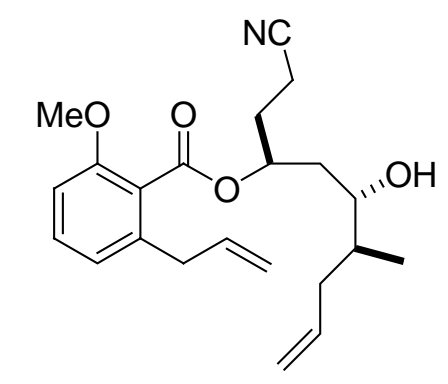

27 


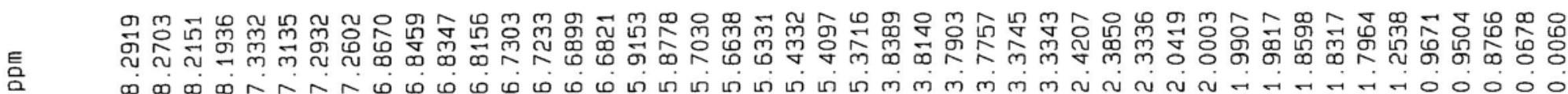
め ம $\underbrace{L}_{m}$

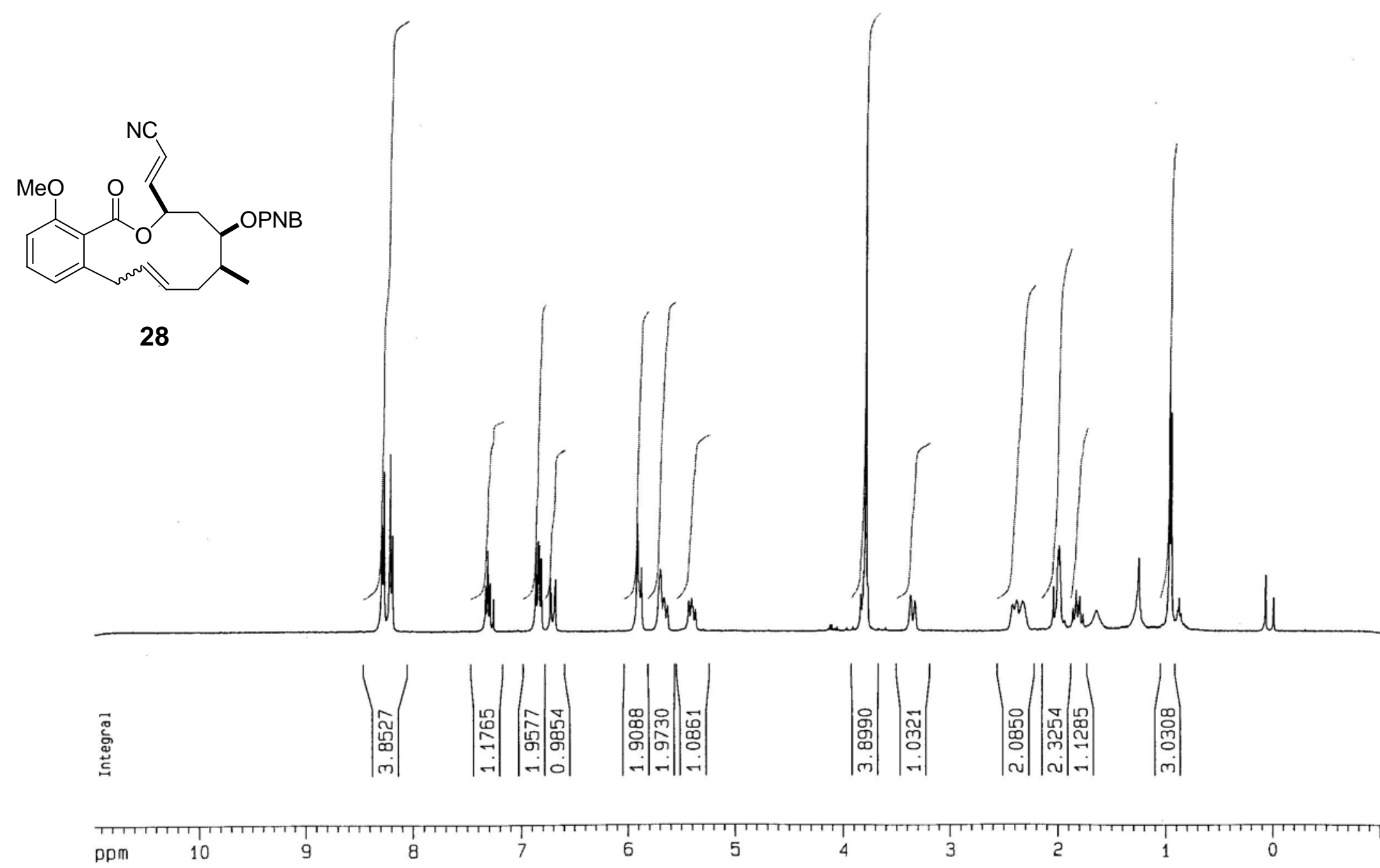




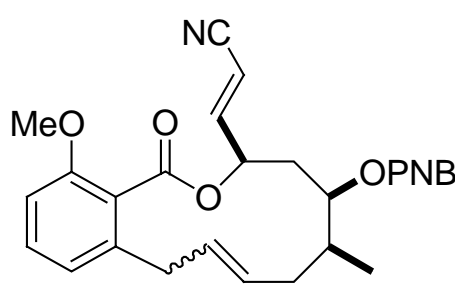

28

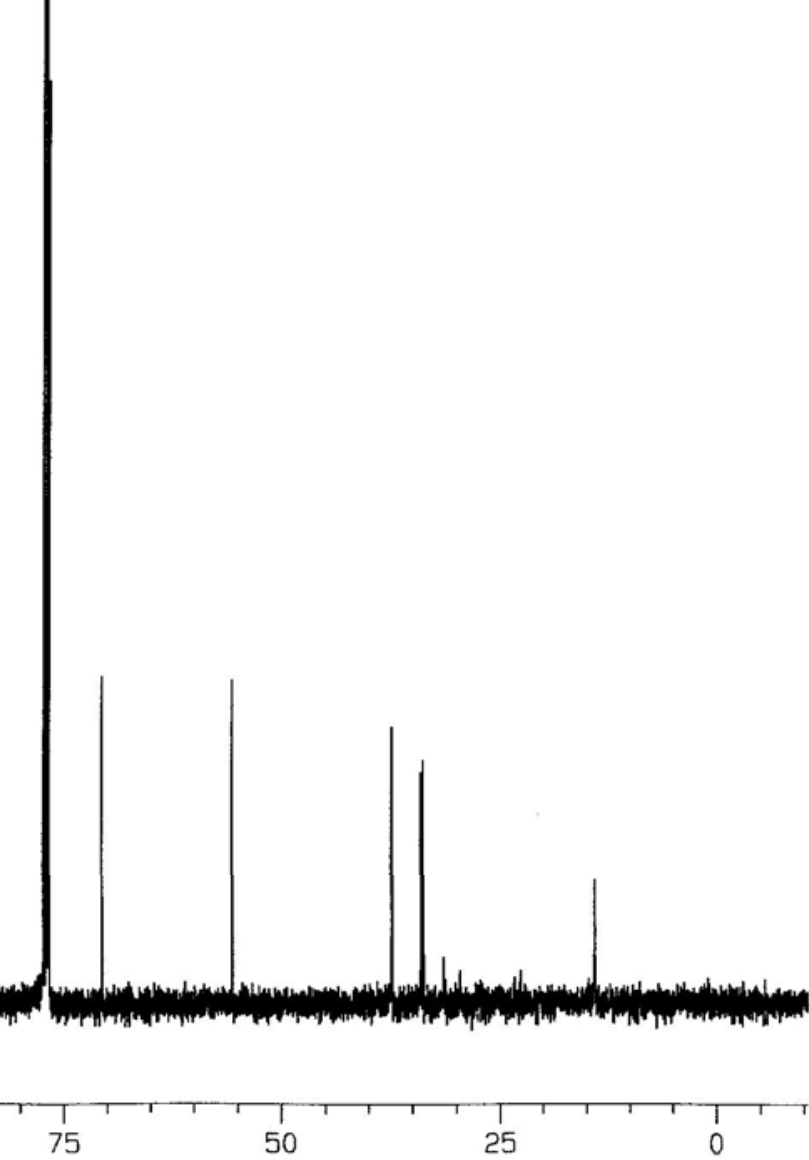




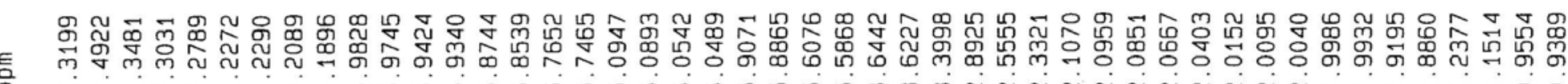
б

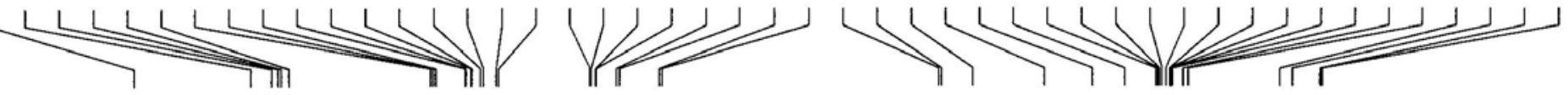<smiles>CC1C=CCc2cccc(O)c2C(=O)OC(C=CC#N)CC1O[Sb]</smiles>

29

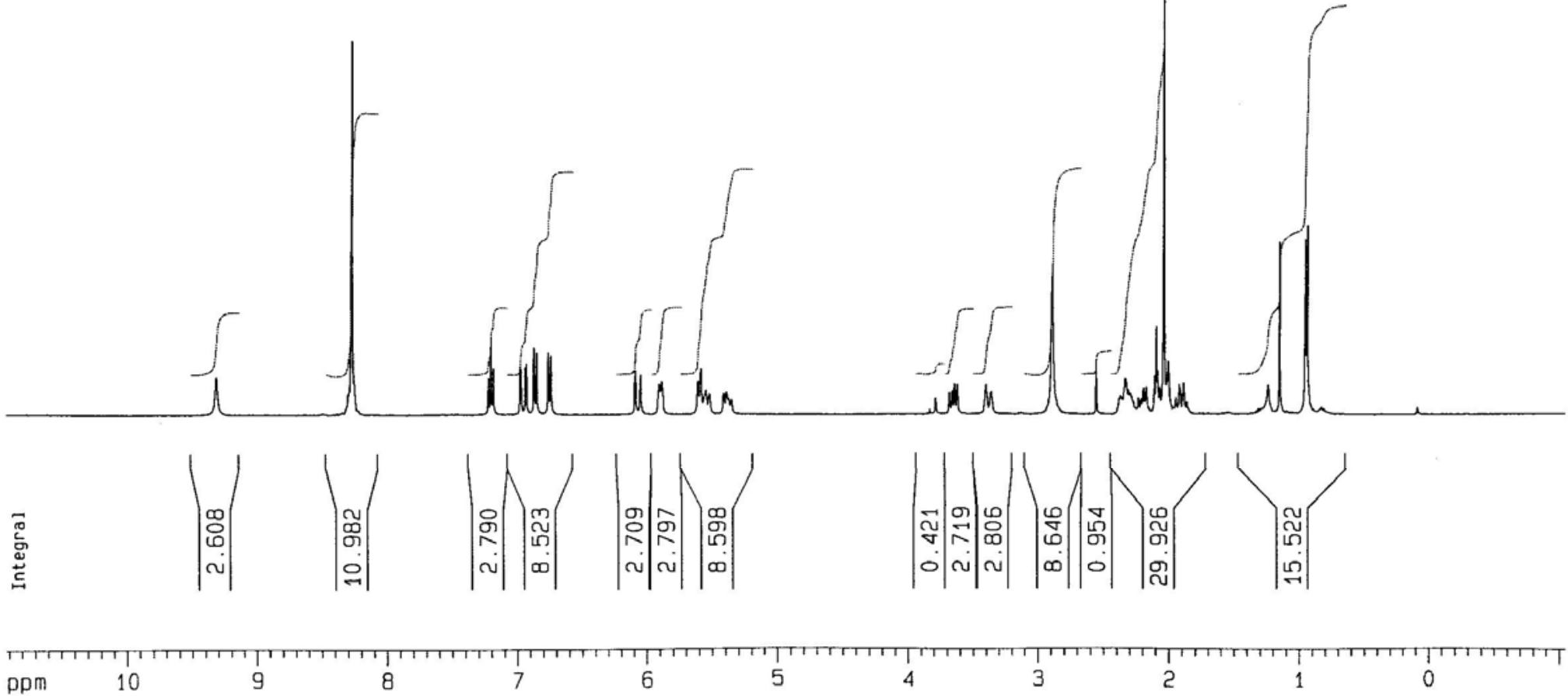




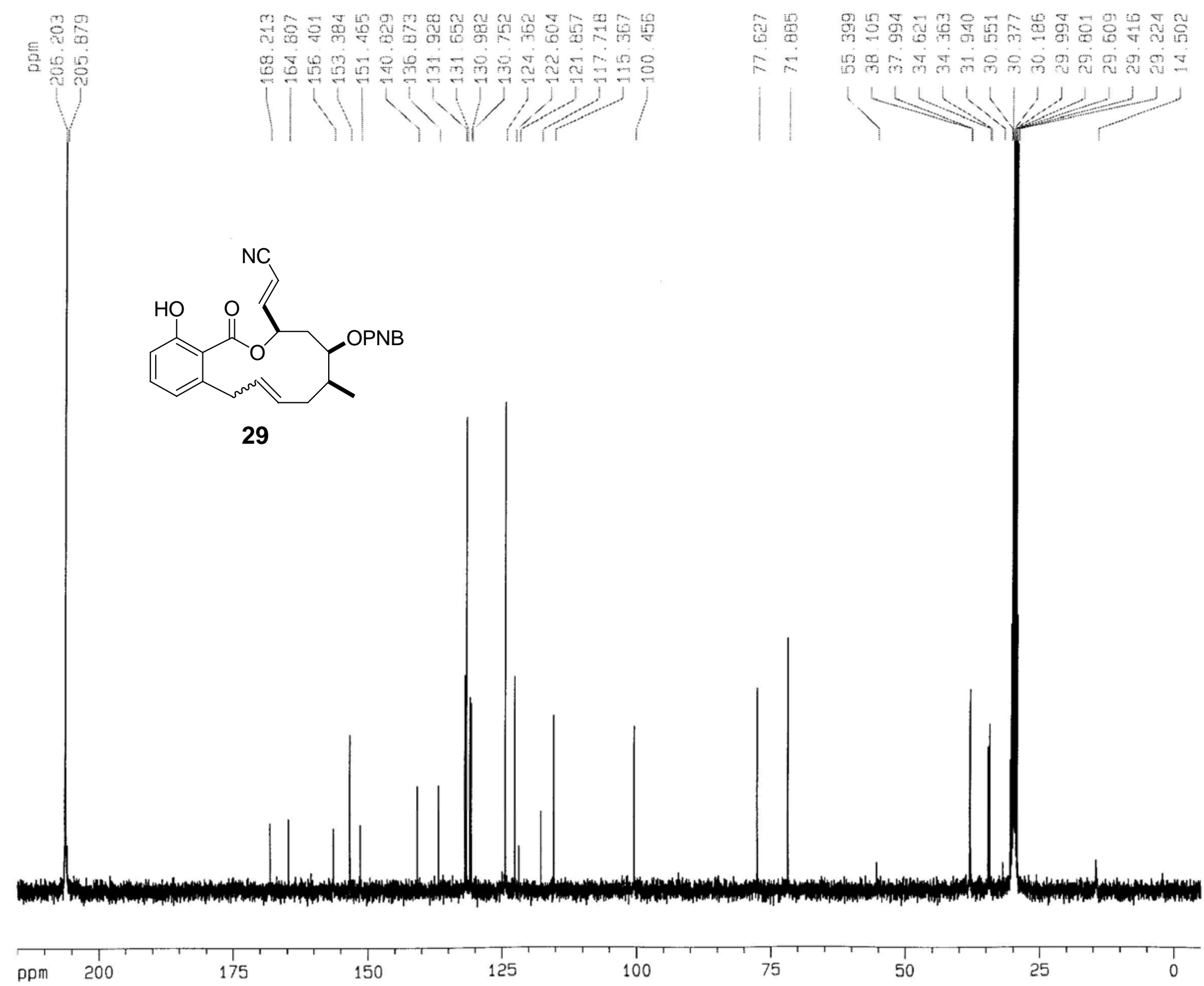



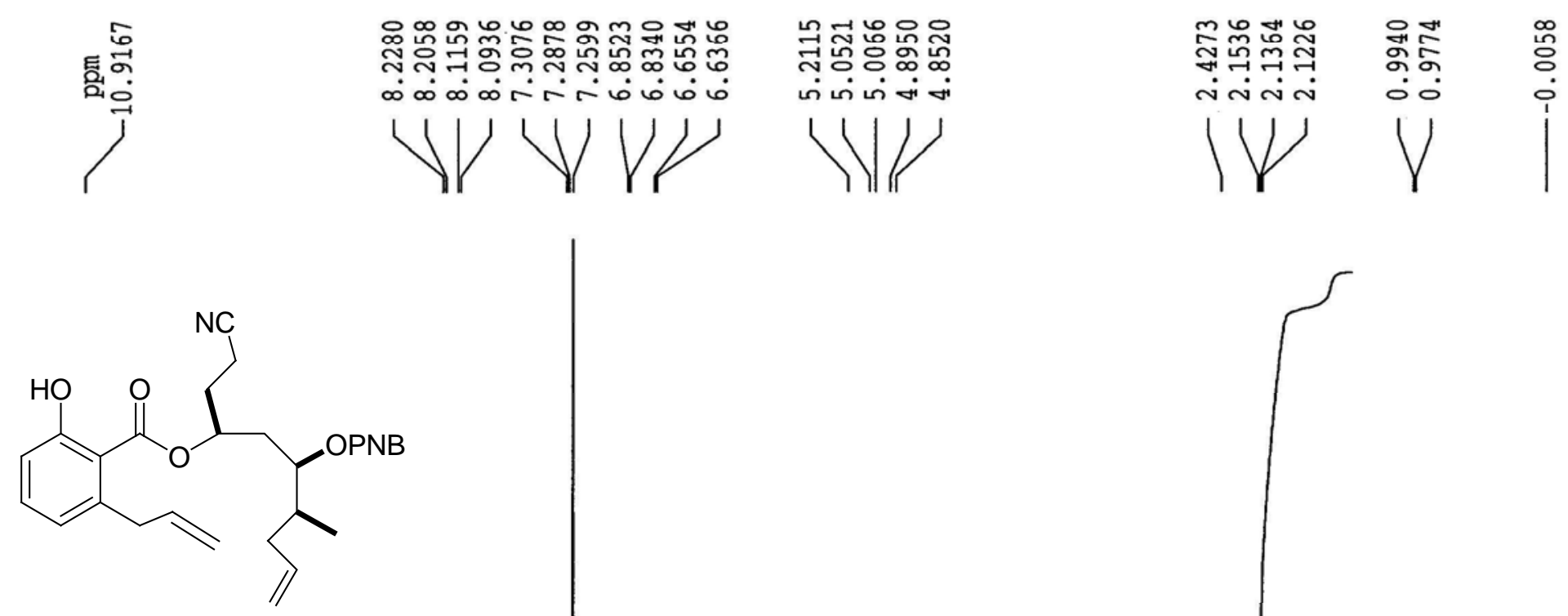

30

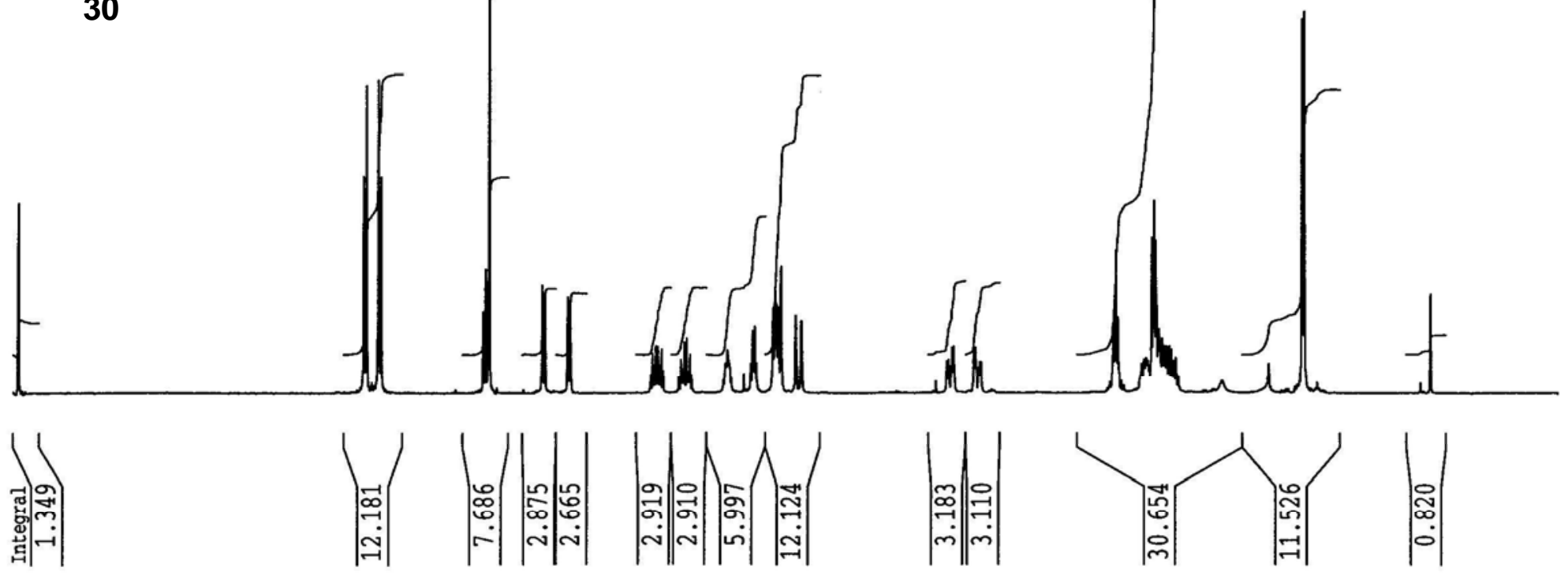

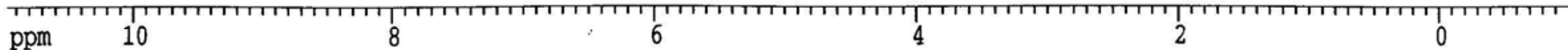



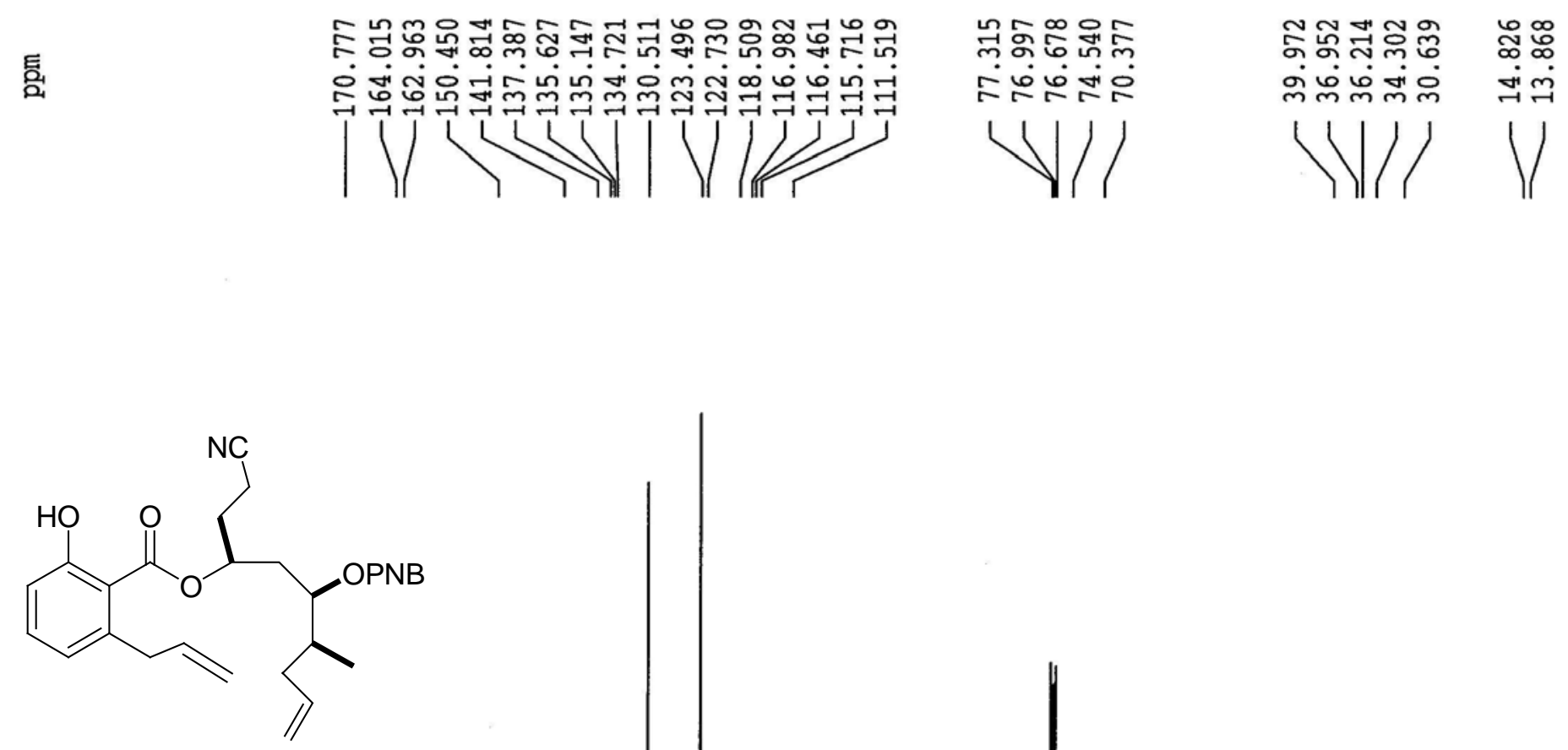

30

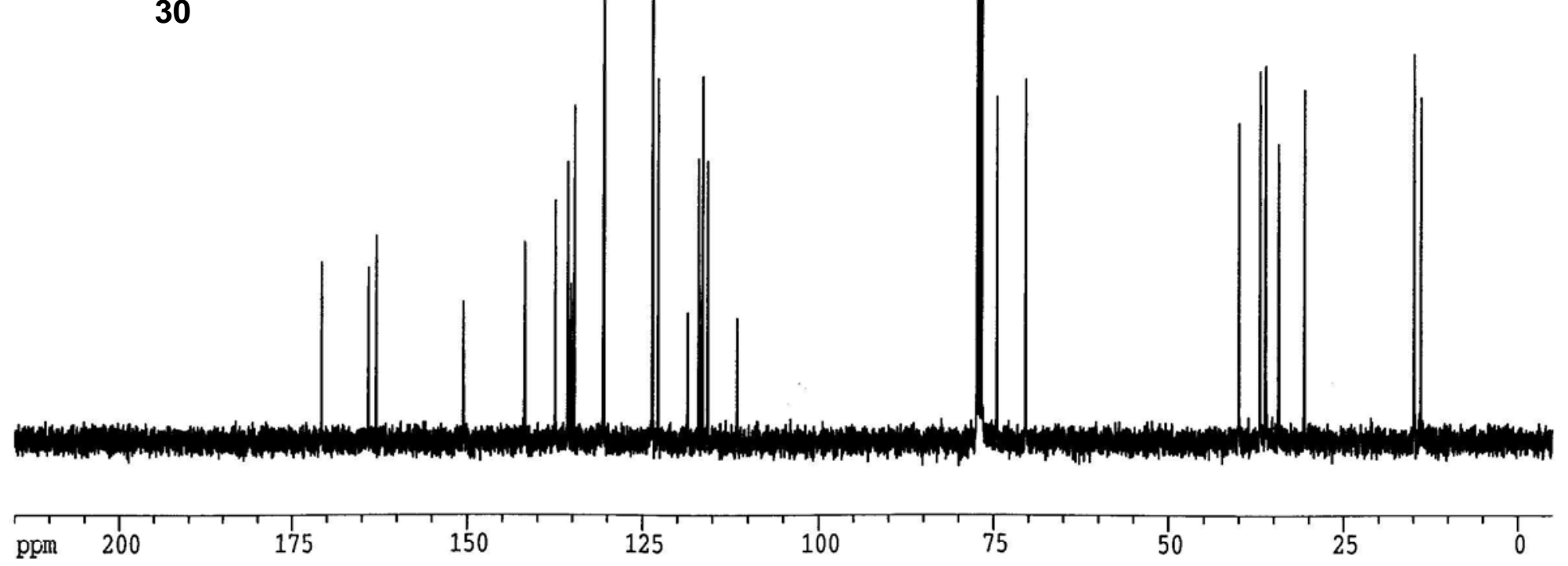




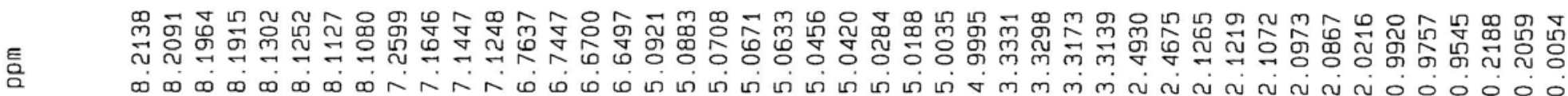

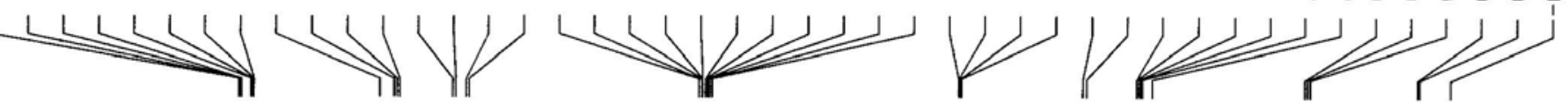<smiles>C=CCc1cccc(OC(F)(F)F)c1C(=O)OC(CCC#N)CC(O[Na])C(C)CC=C</smiles>

31

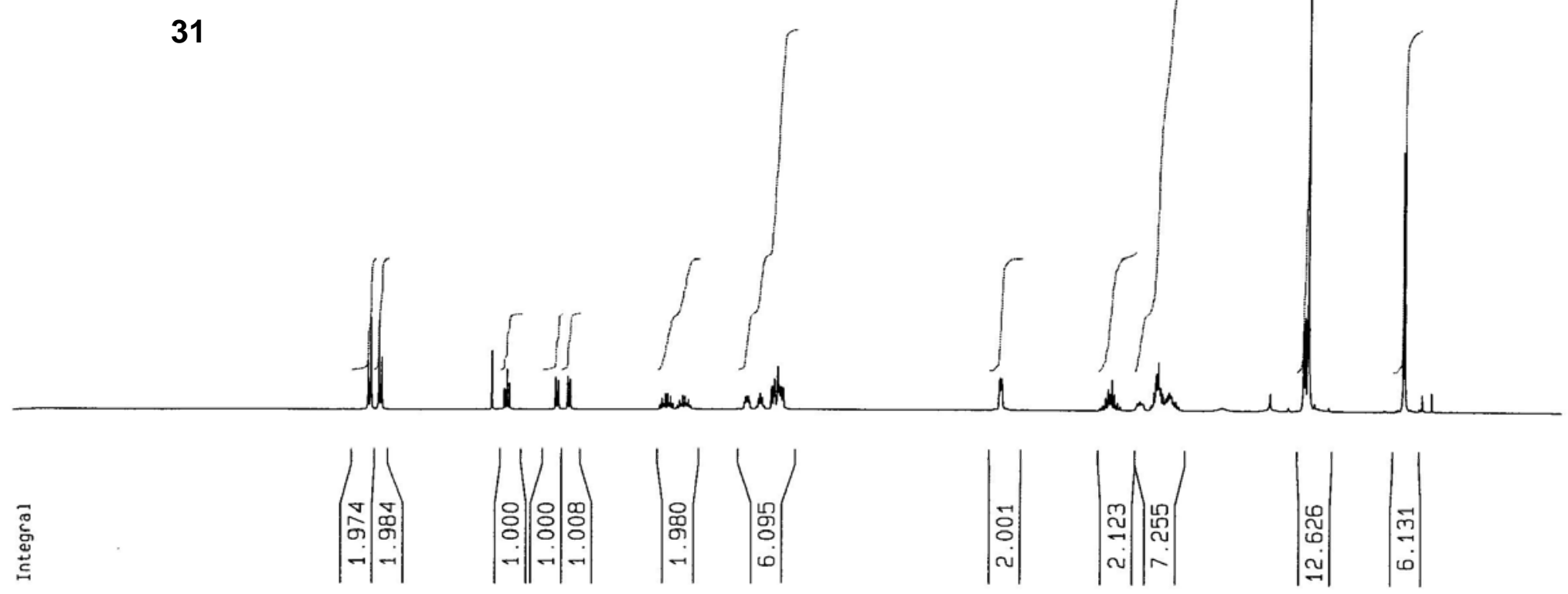



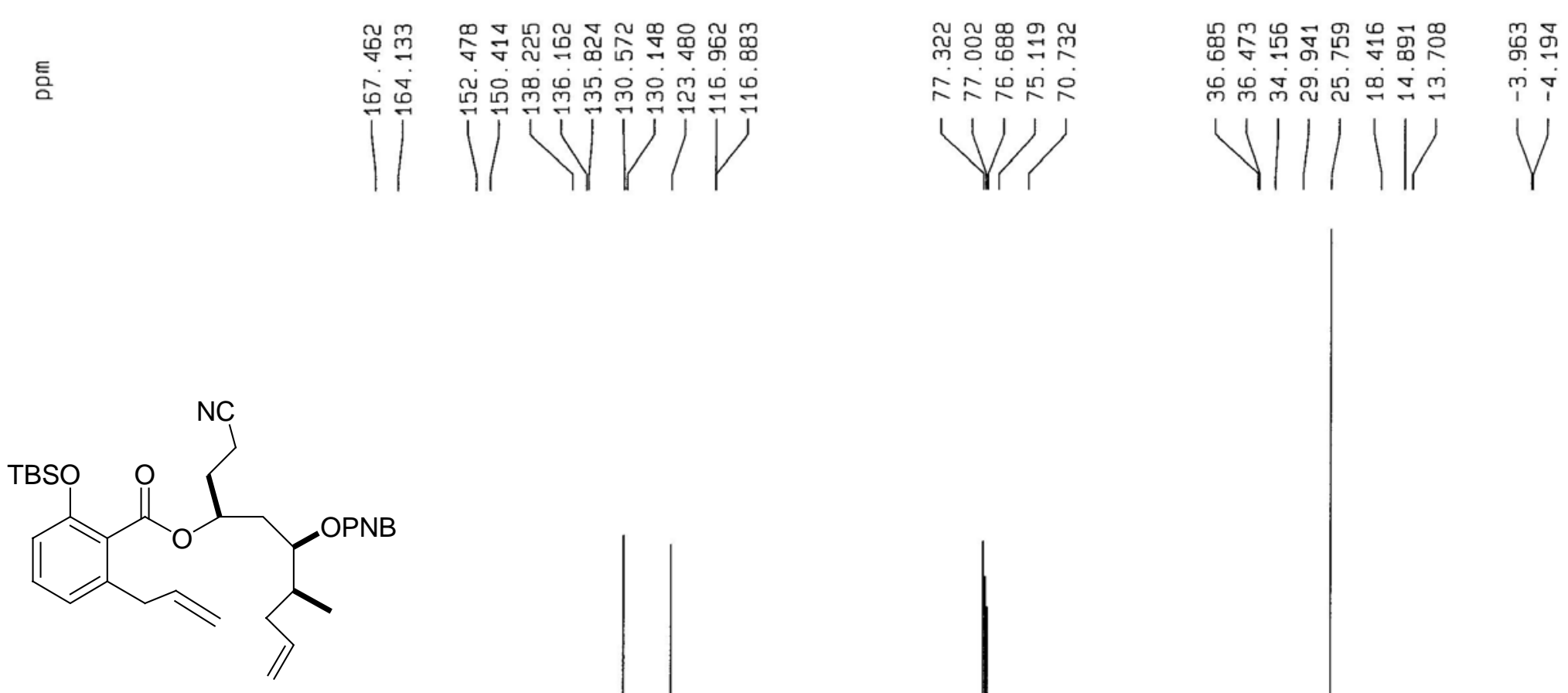

31 


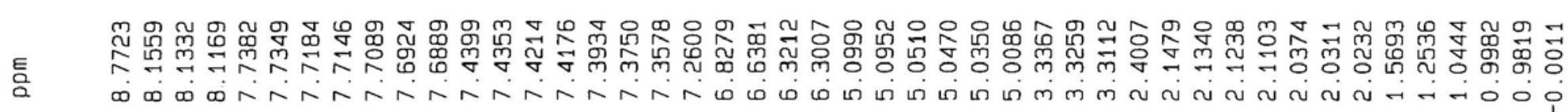
$\underbrace{L}_{1}+\mid$

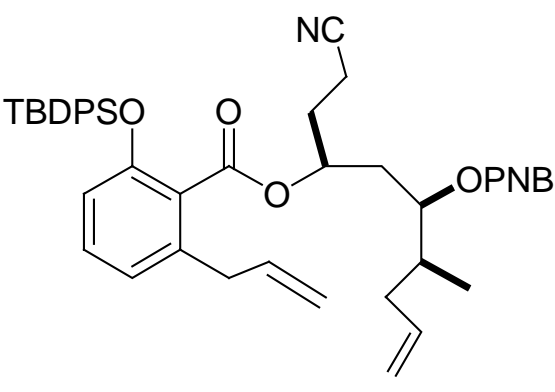

32

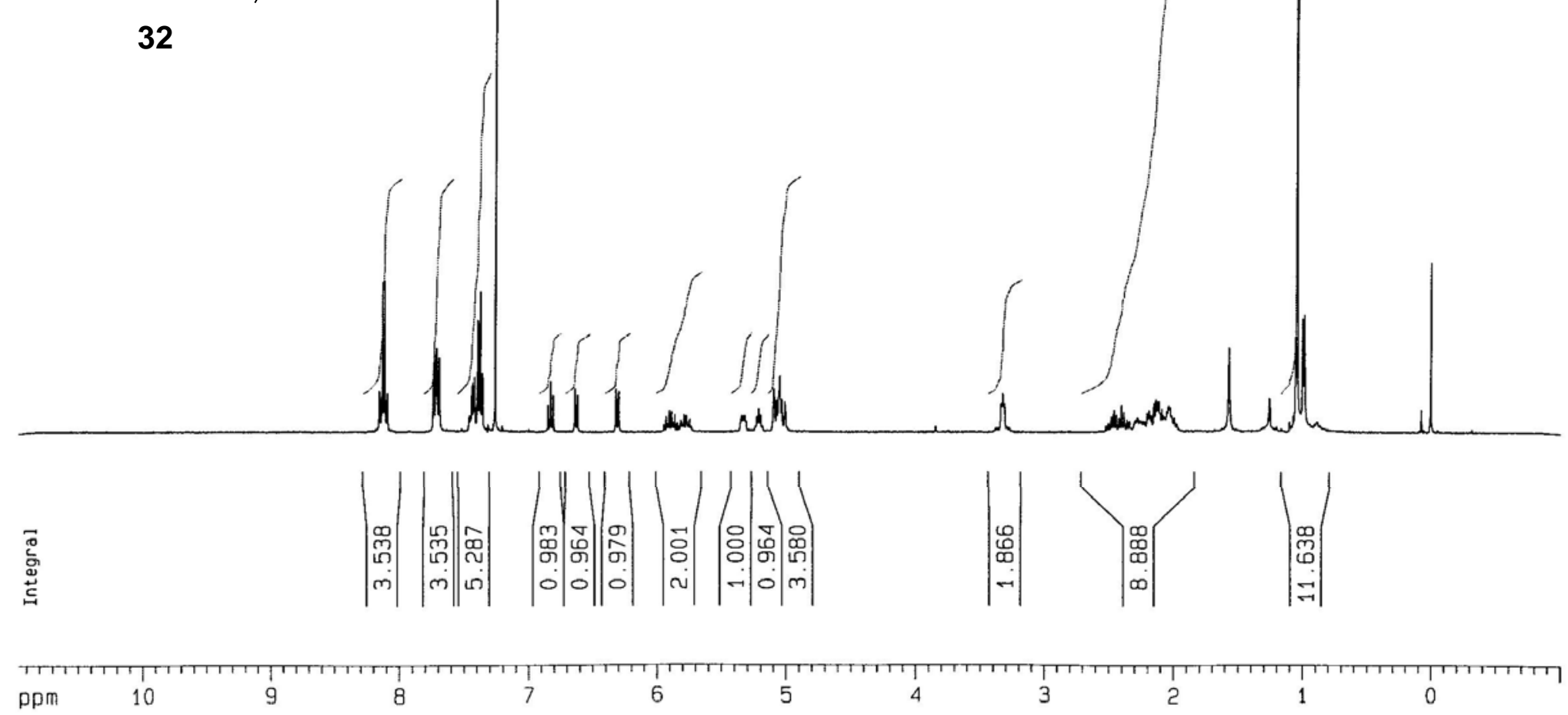




$$
1
$$




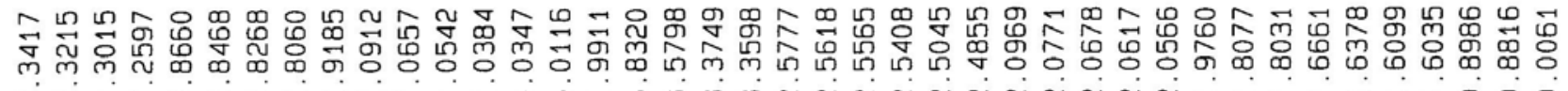
A N $\wedge$ ம
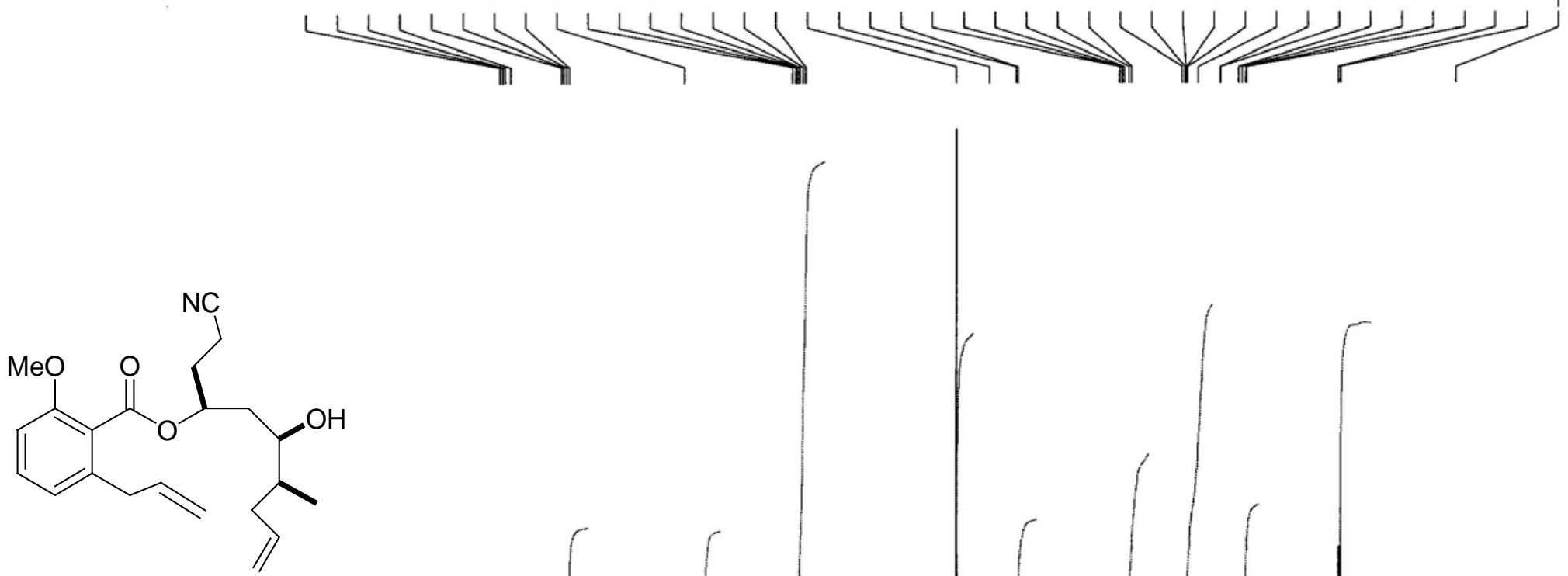

33

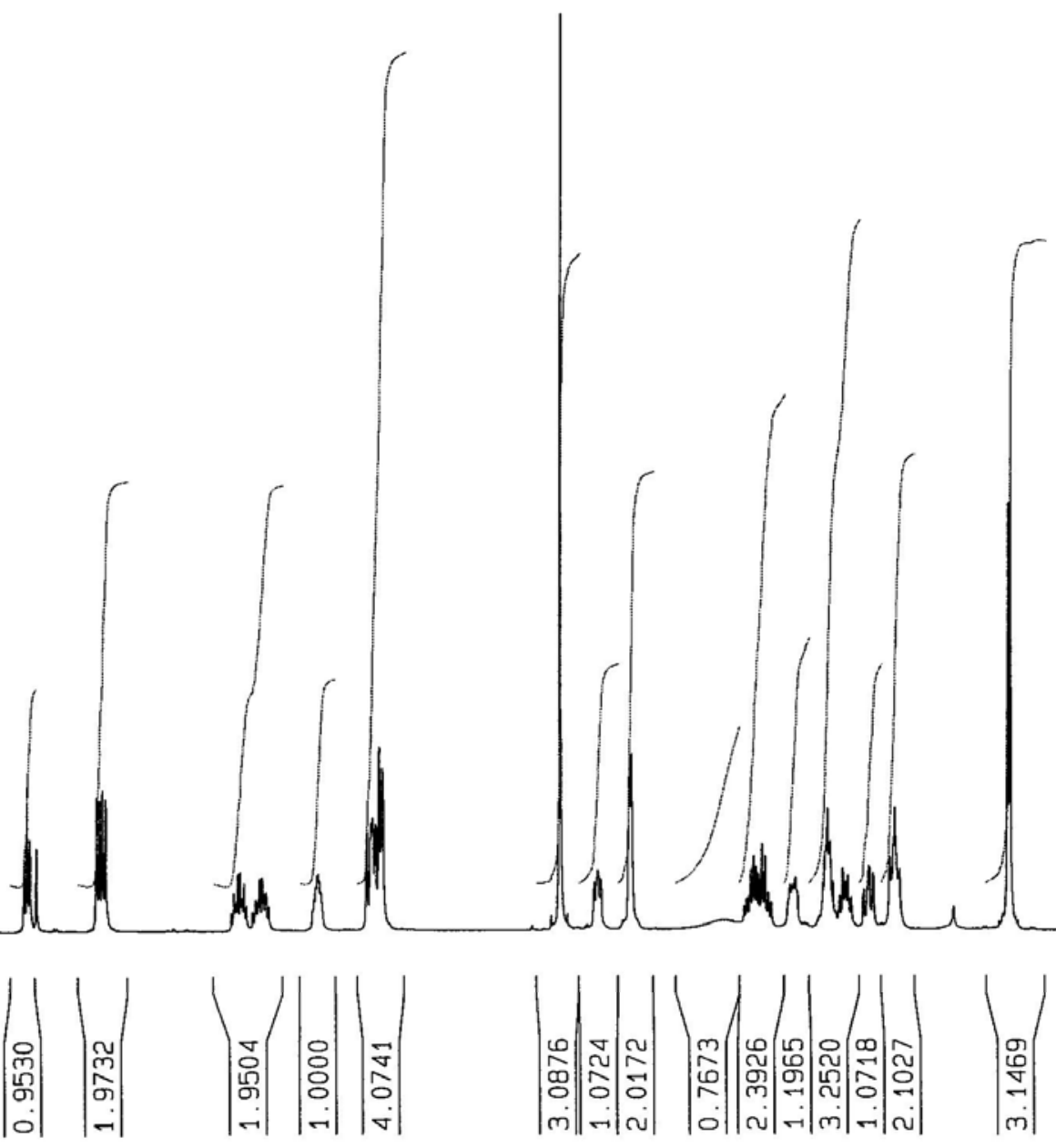




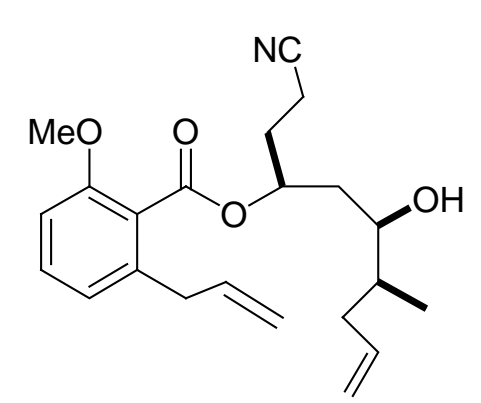

33

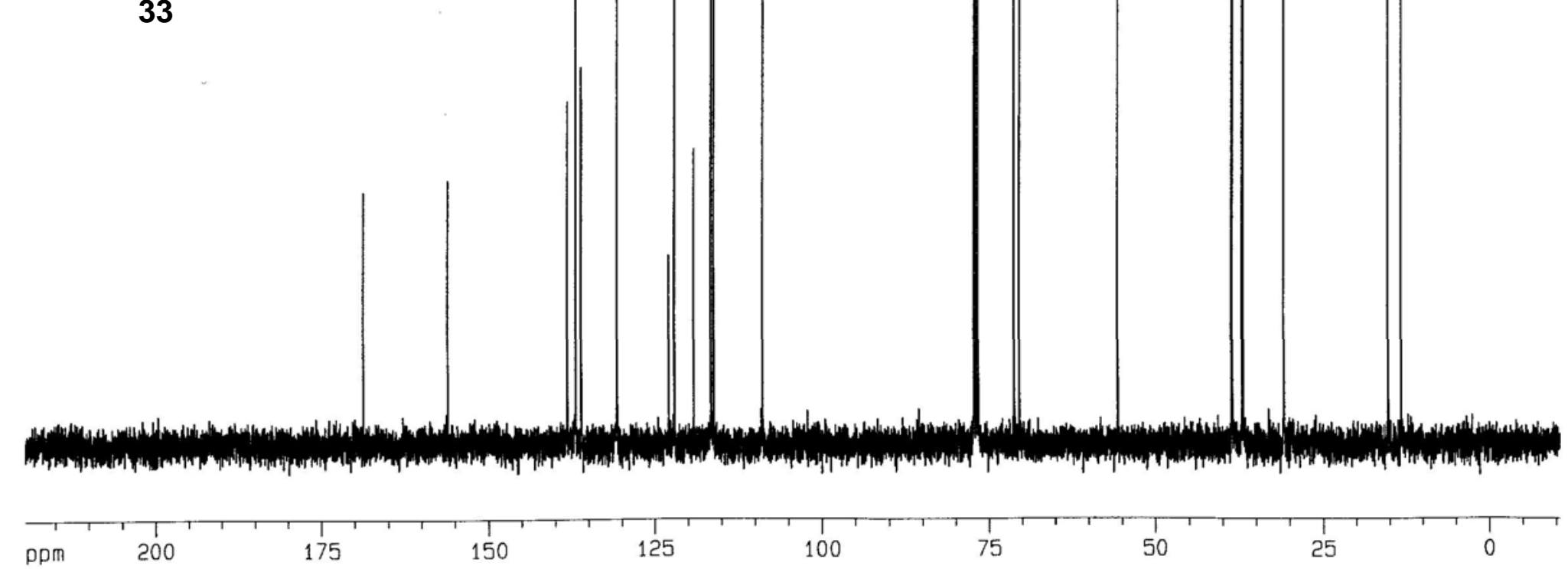




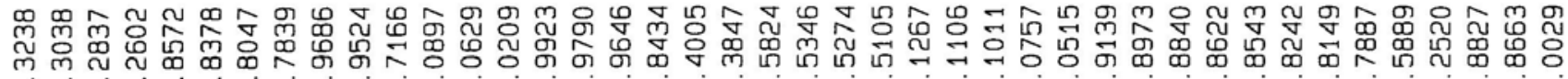
ヘ $\sim \sim$ ம
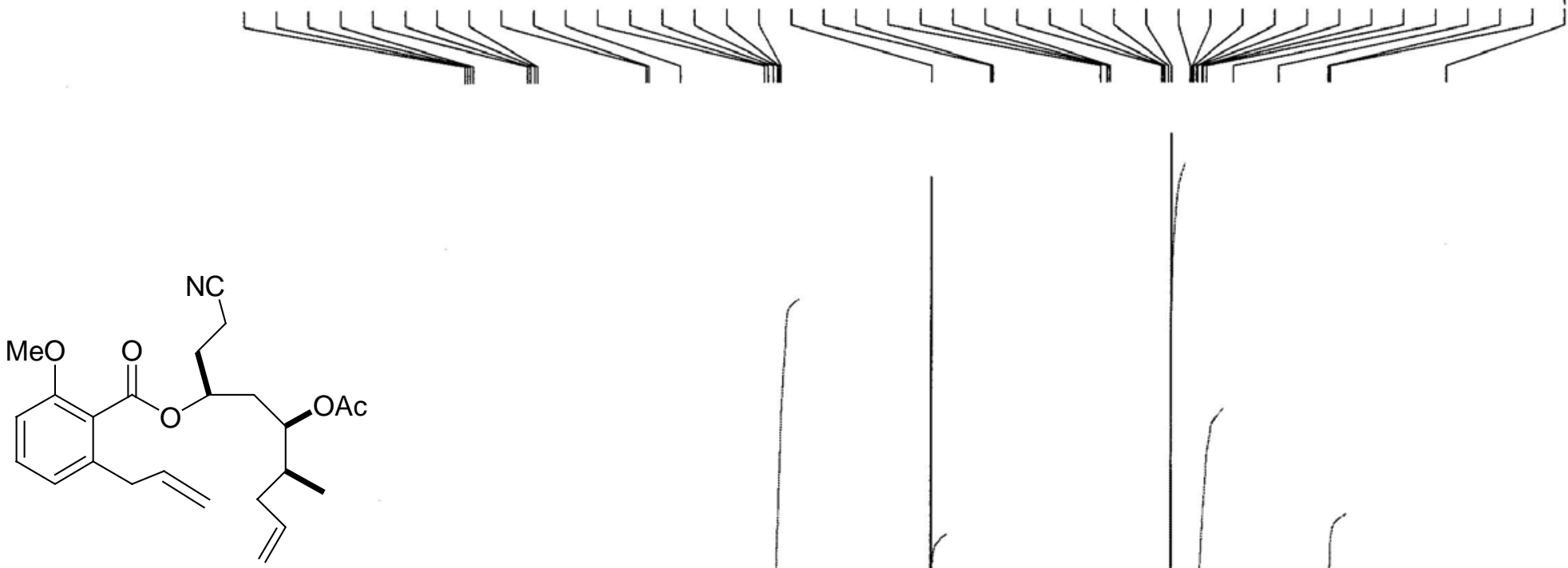

34

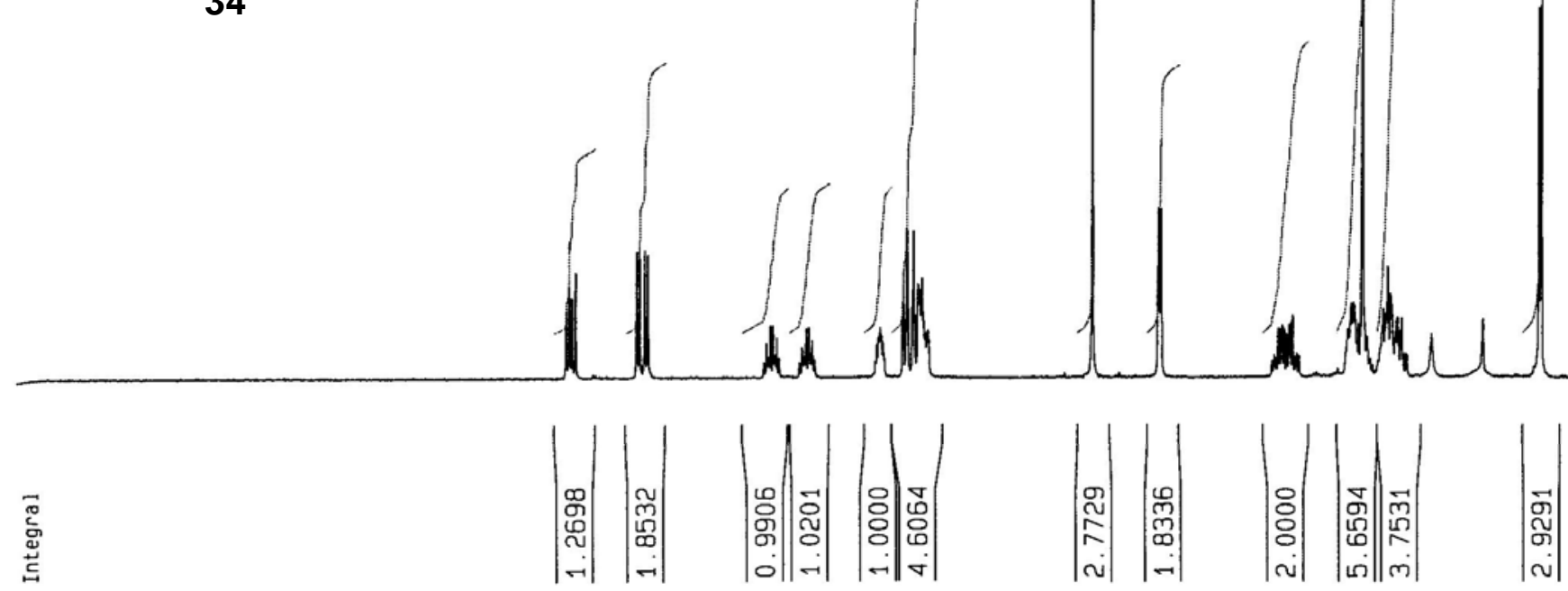


言
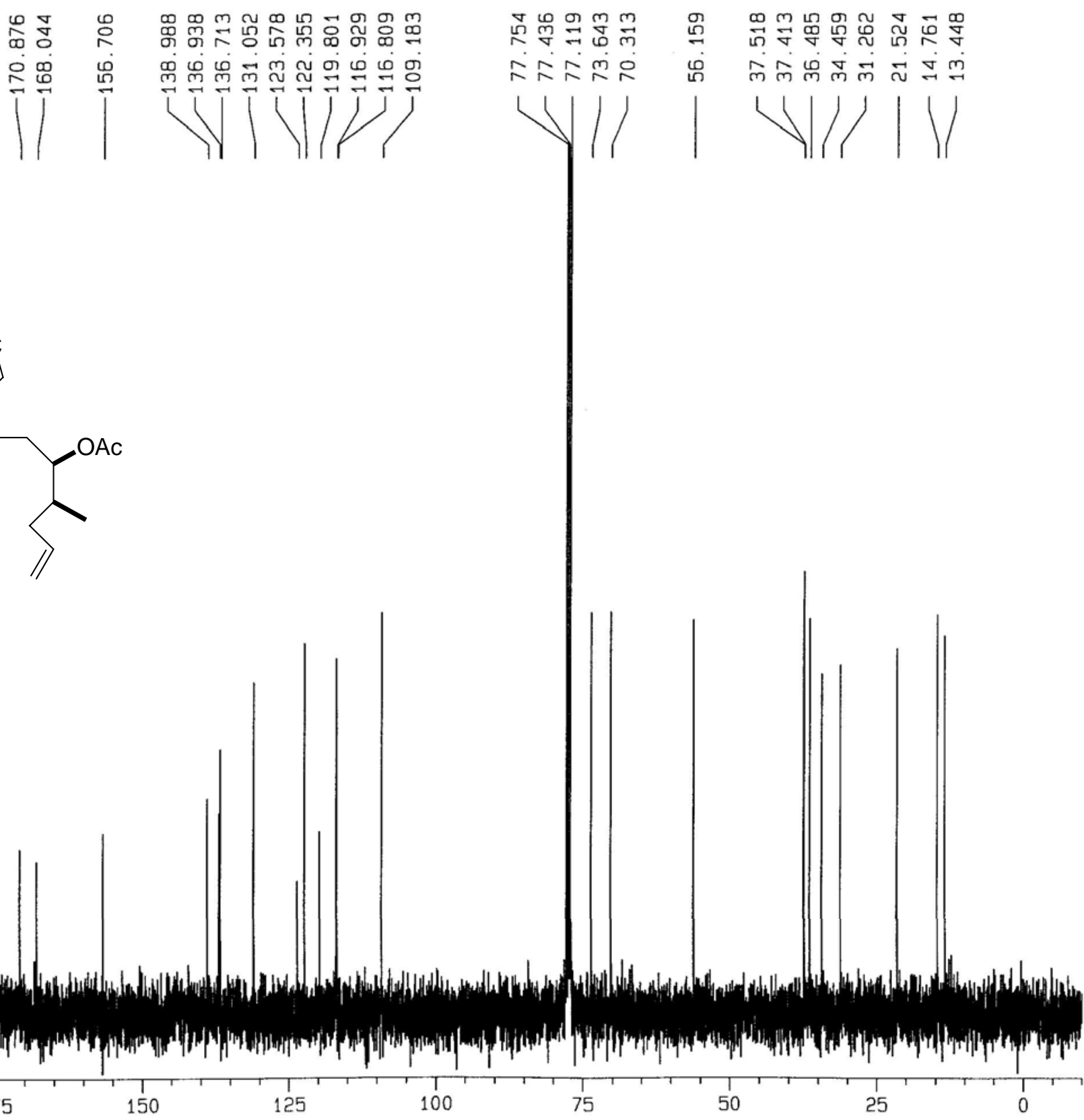

S53 


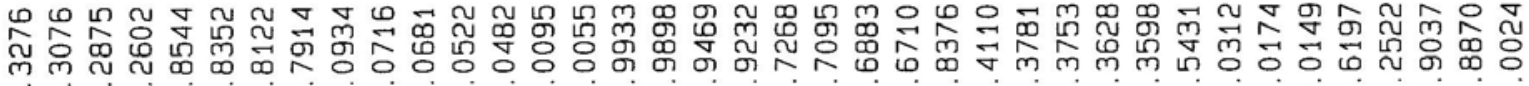

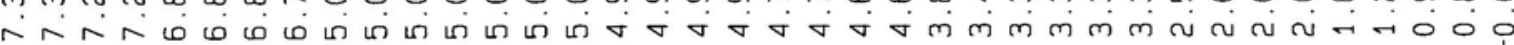
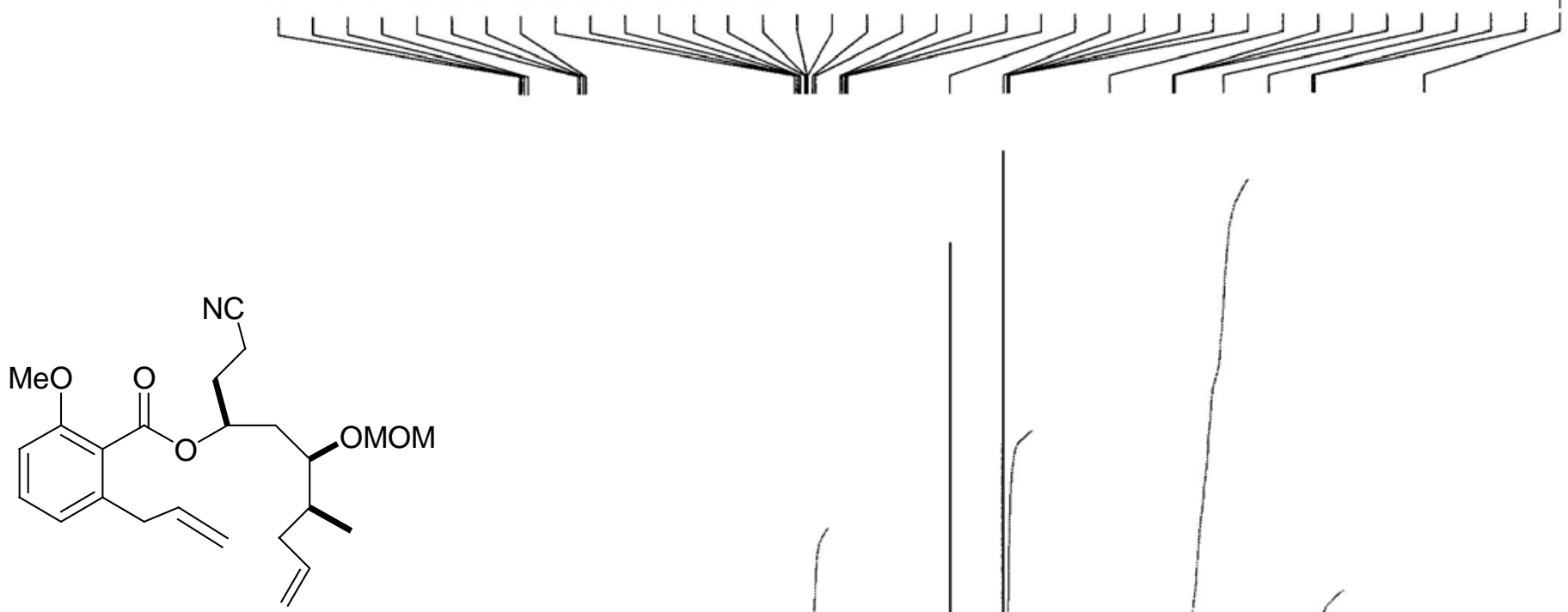

35

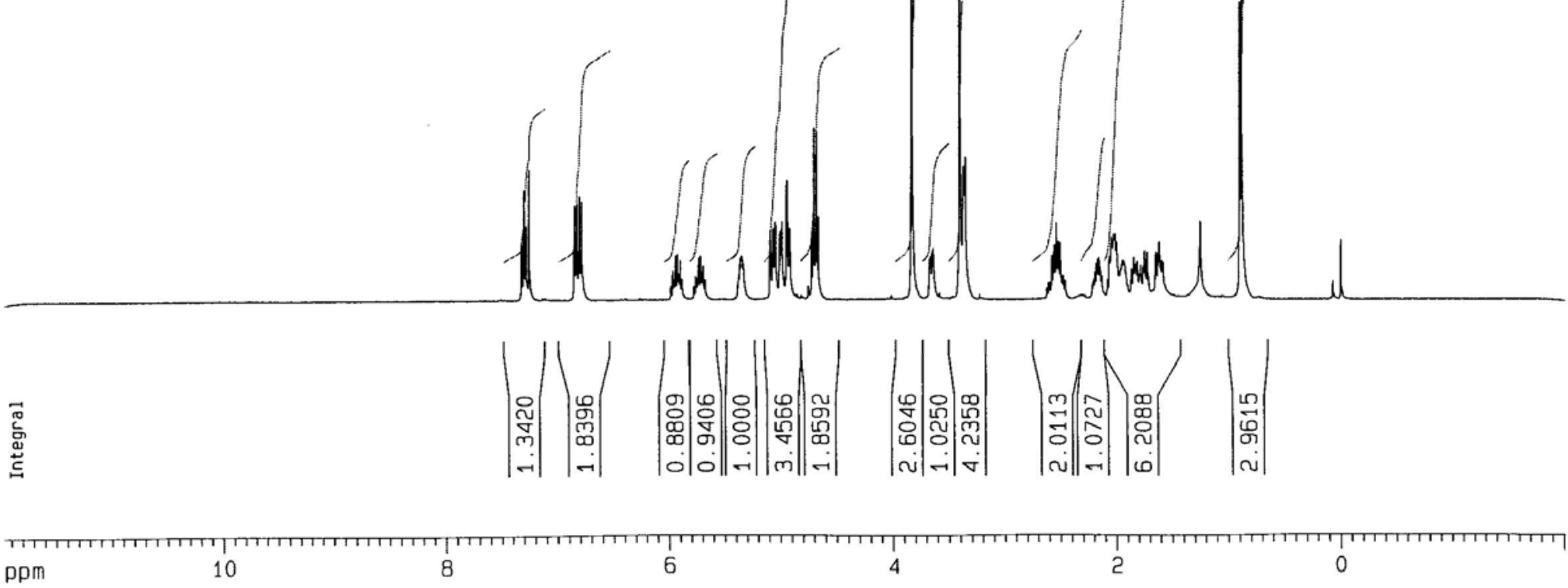




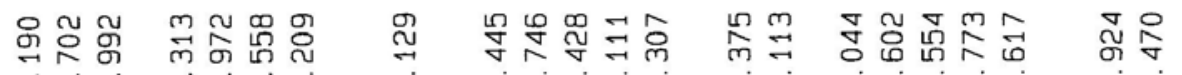

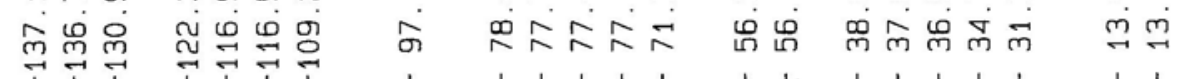

VIVI I Y' Y WI/ V

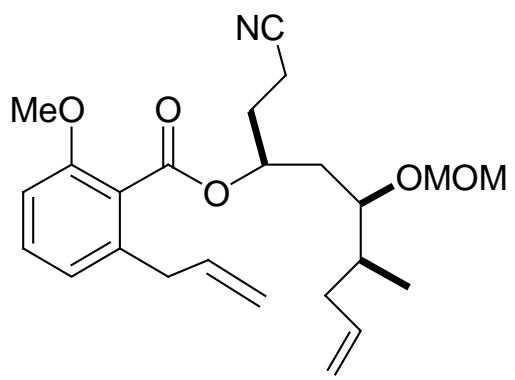

35

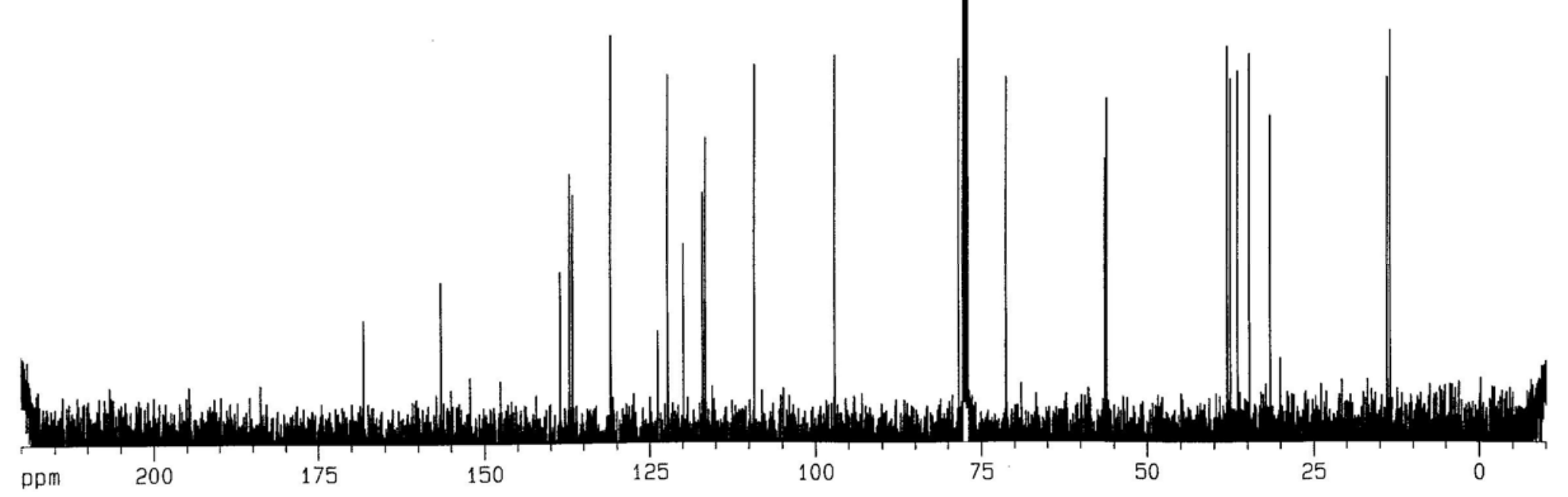




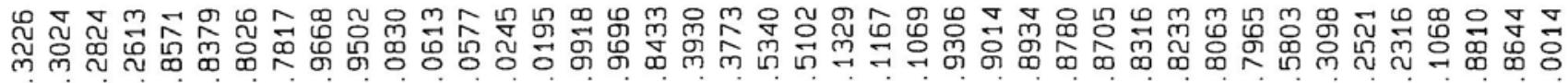

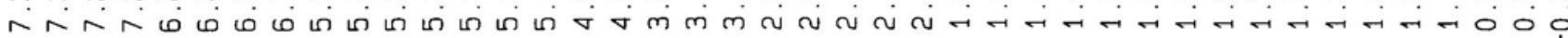
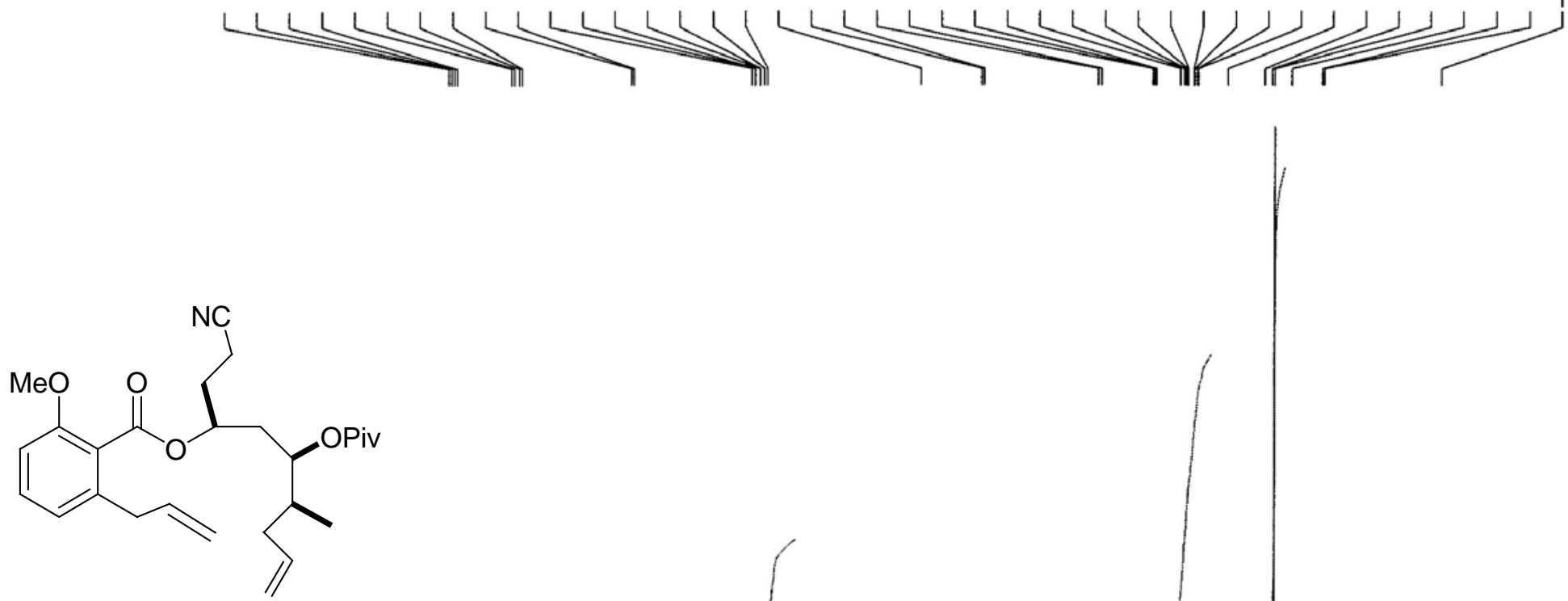

36

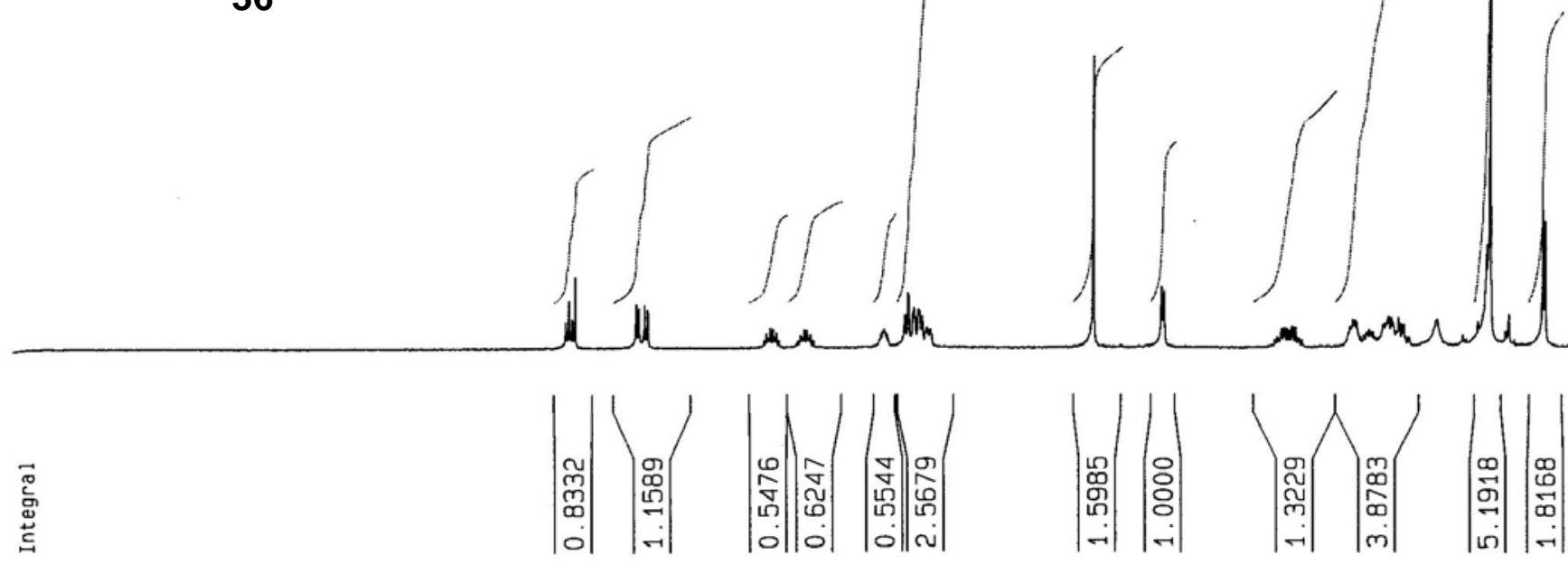




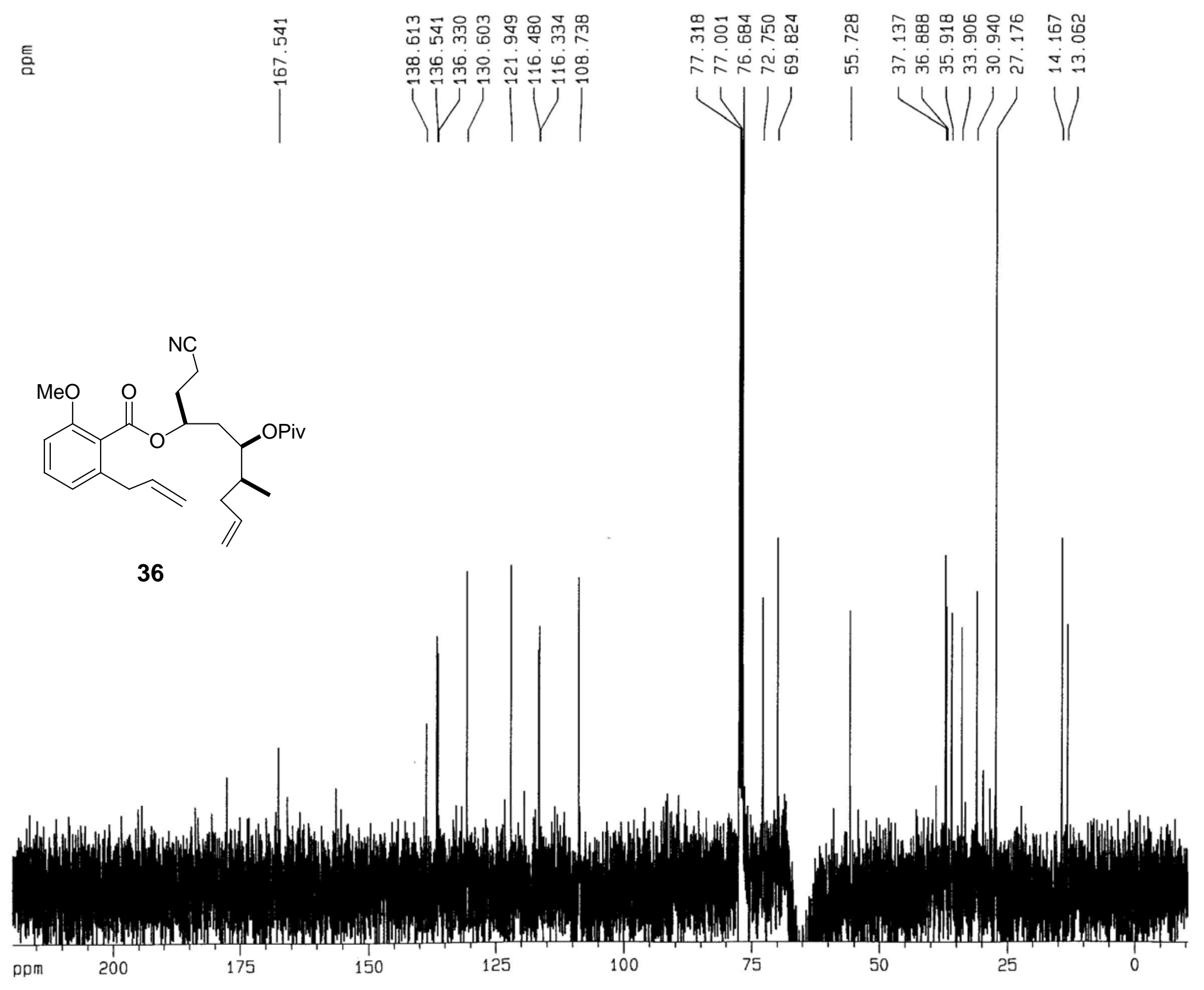



ヘ $\wedge$ ம

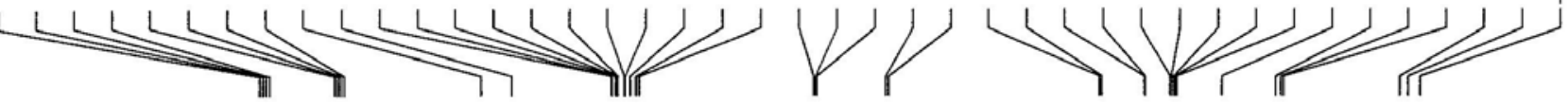

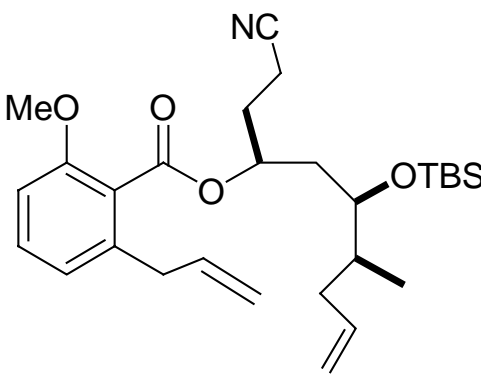

37

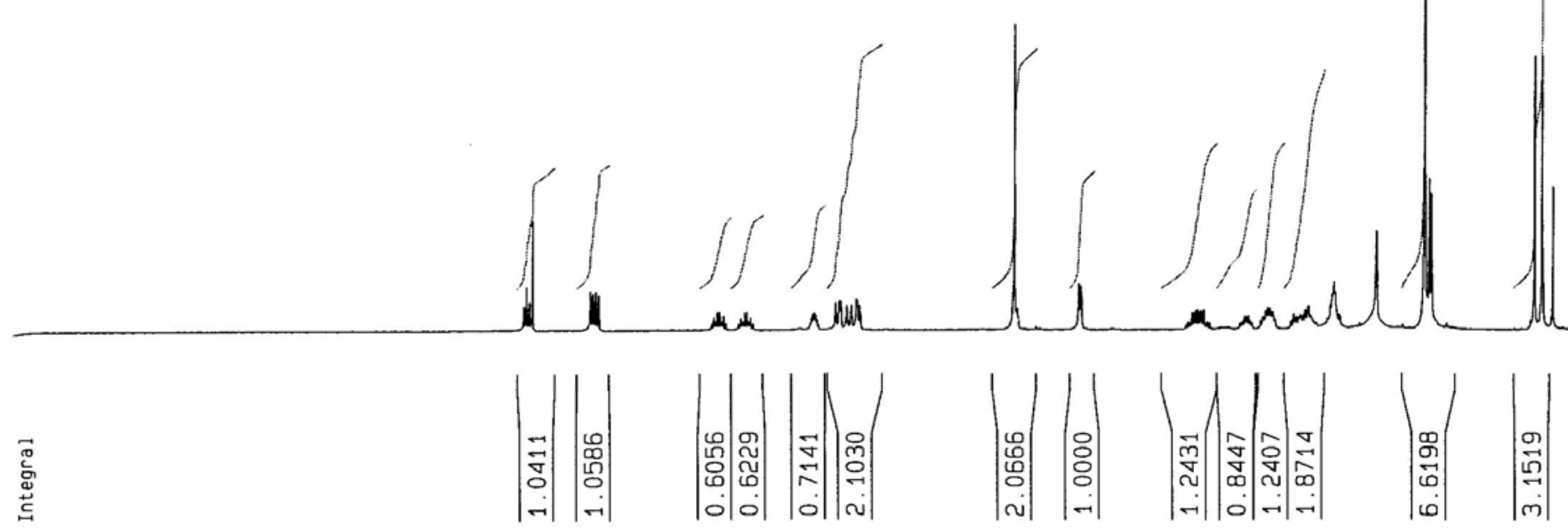




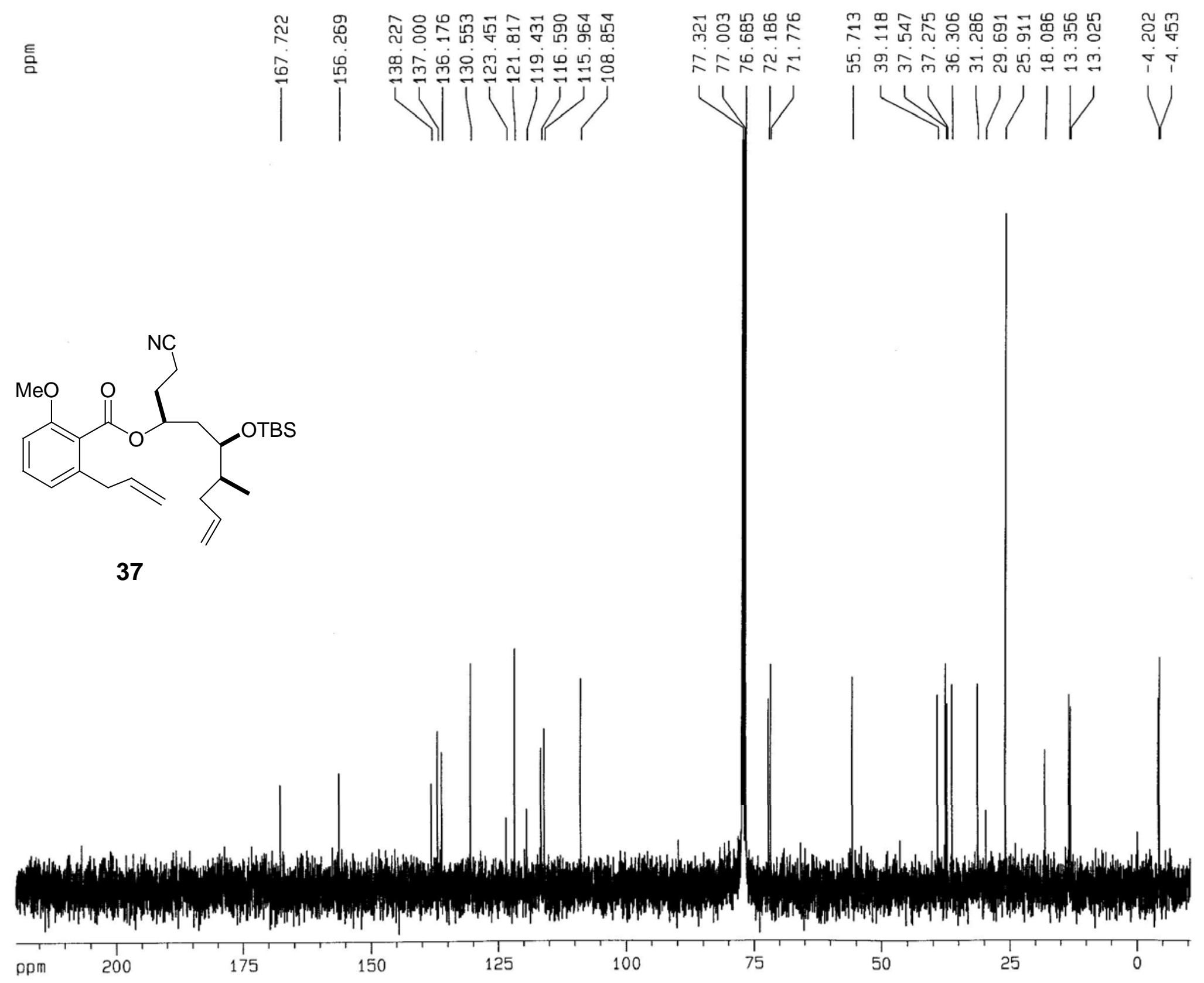




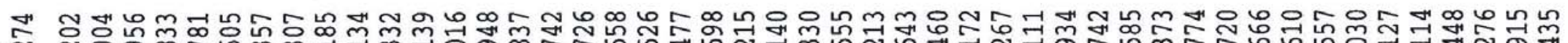
대 긍에 मी
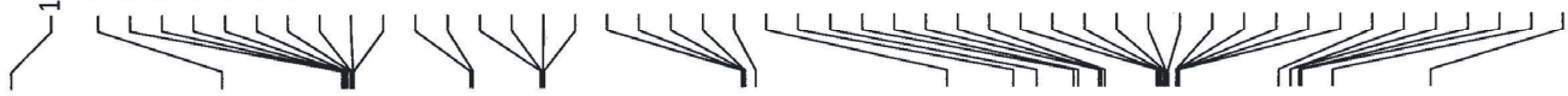

HPLC results (Silica gel 60):

Gradient ethyl acetate in hexanes $0 \%$ ethyl acetate to $50 \%$ ethyl acetate (30 minutes)

Peak 1 (29.9 min): 75.1\%

Peak 2 (32.7 min): $24.9 \%$

NMR integration results:

$E: Z 1: 2$

Ratio determined based on HPLC

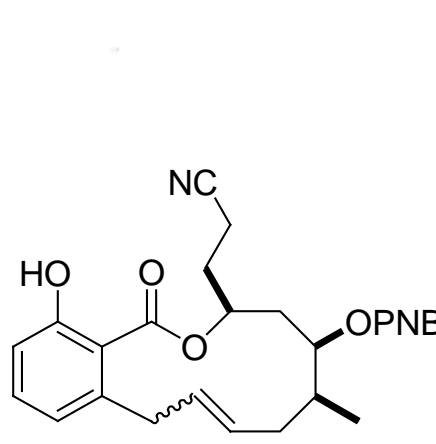

38
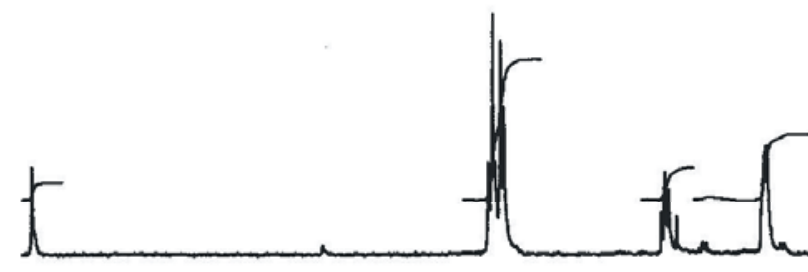

E-isomer
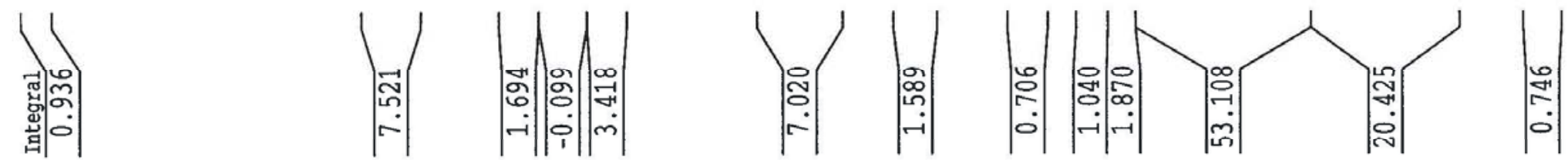


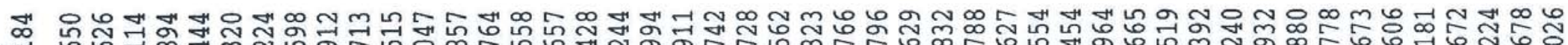

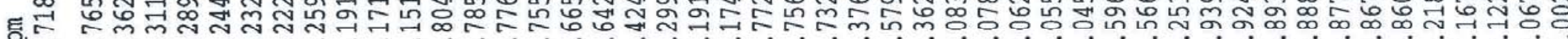
न
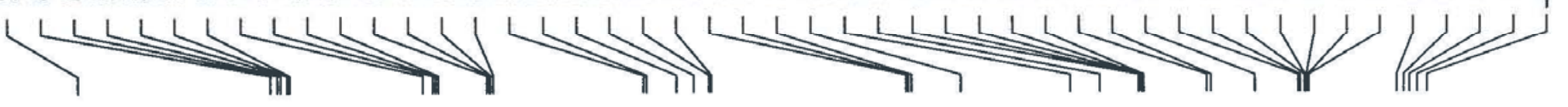

HPLC results (Silica gel 60):

Gradient ethyl acetate in hexanes

$0 \%$ ethyl acetate to $30 \%$ ethyl acetate

(30 minutes)

Peak 1 (19.9 min): $12.0 \%$

Peak 2 (20.9 min): 88.0\%

NMR integration results:

$E: Z 7: 1$

Ratio determined based on NMR and HPLC
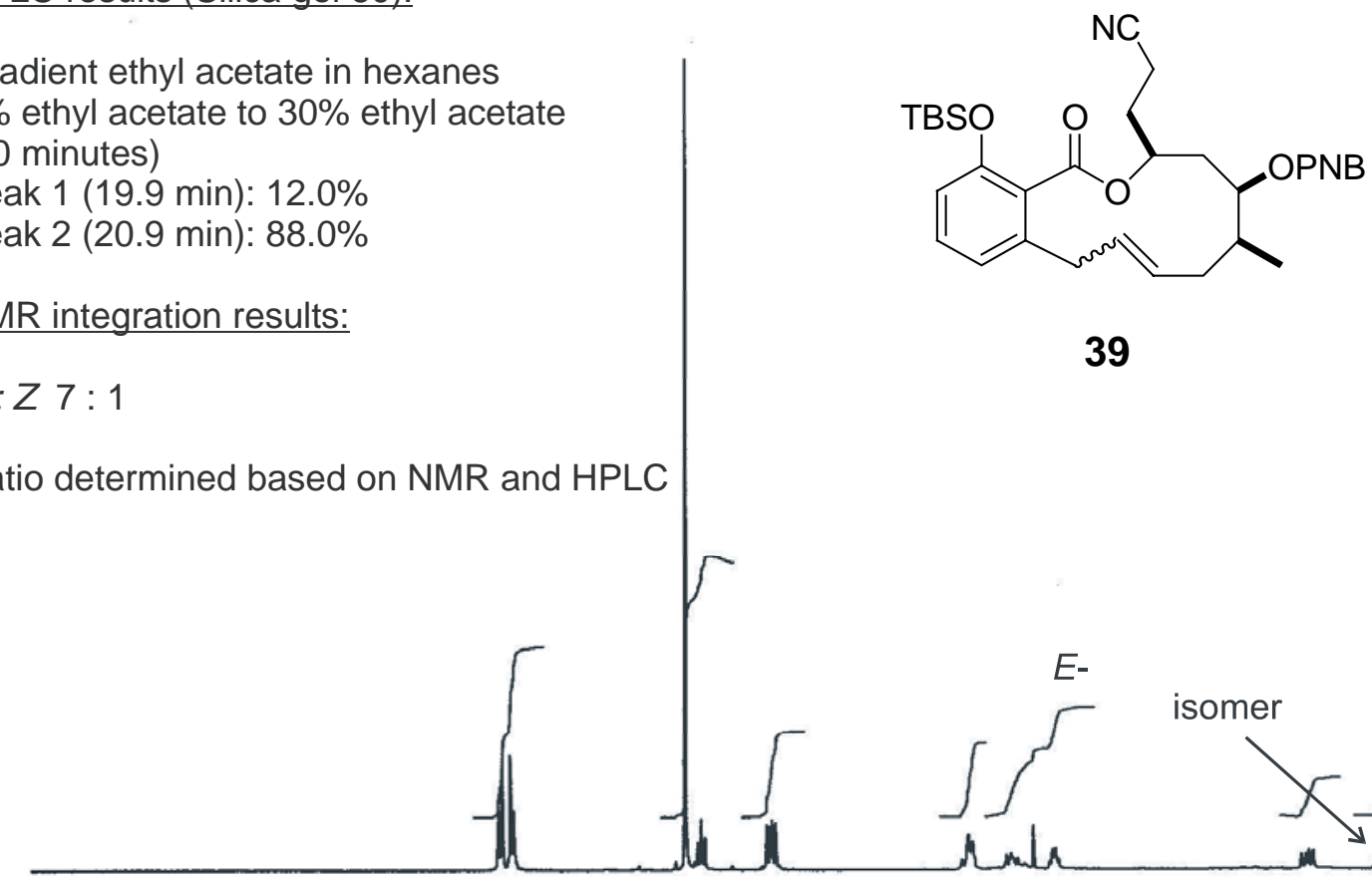

39

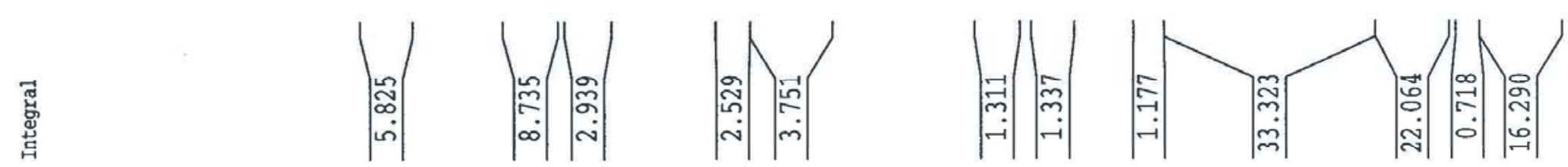

ppm 10

8

6

4

2

0 


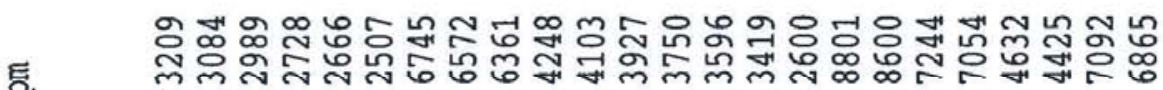 m $\longrightarrow$}

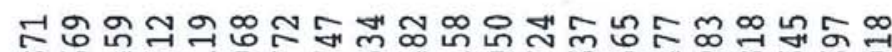

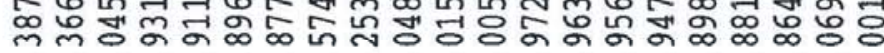
in i-i

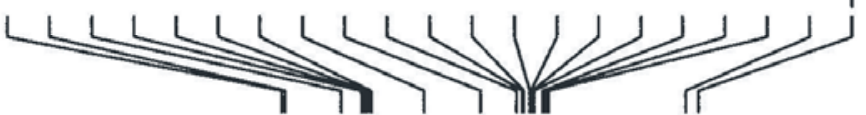

HPLC results (Silica gel 60):

Gradient ethyl acetate in hexanes

$0 \%$ ethyl acetate to $30 \%$ ethyl acetate

(30 minutes)

Peak 1 (22.3 min): $74.4 \%$

Peak 2 (23.6 min): $25.6 \%$

NMR integration results:

$E: Z 3: 1$

Ratio determined based on NMR and HPLC
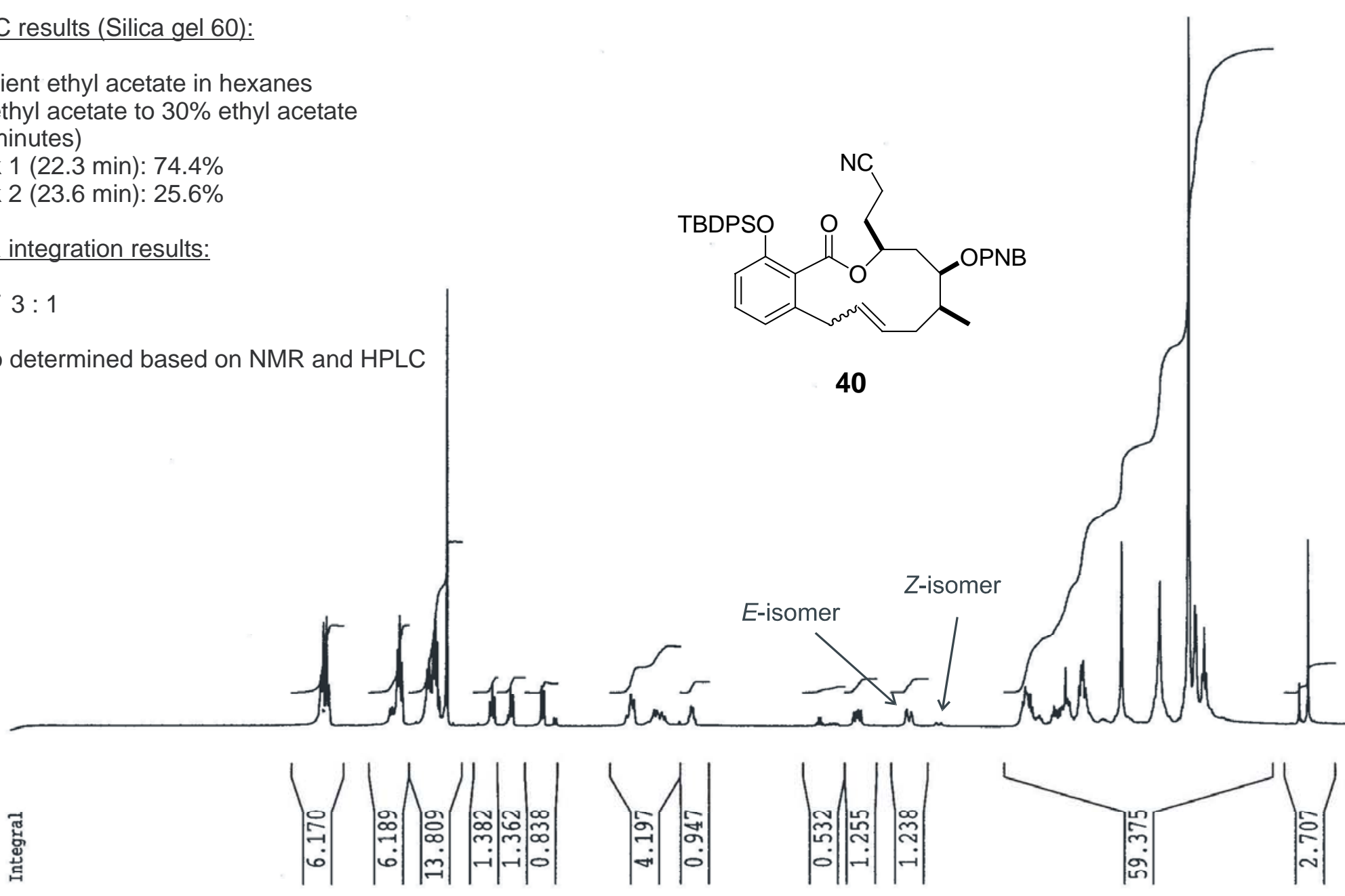

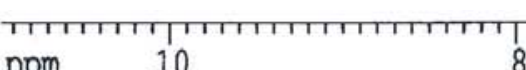

ppm

10

6

4

2

0 


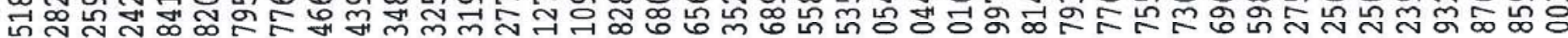

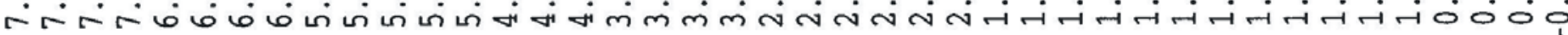

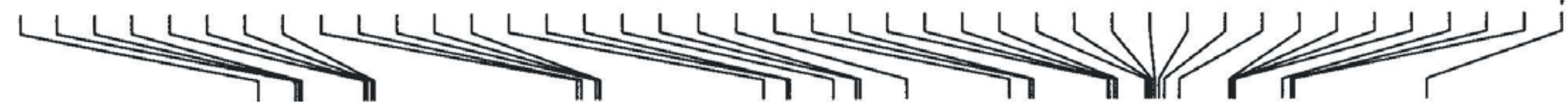

\section{HPLC results (Silica gel 60):}

Gradient ethyl acetate in hexanes

$0 \%$ ethyl acetate to $50 \%$ ethyl acetate

(30 minutes)

Peak 1 (33.0 min): $96.7 \%$

Peak 2 (37.5 min): $3.3 \%$

NMR integration results:

\section{$E: Z \mathrm{n} / \mathrm{d}$}

Ratio determined based on HPLC

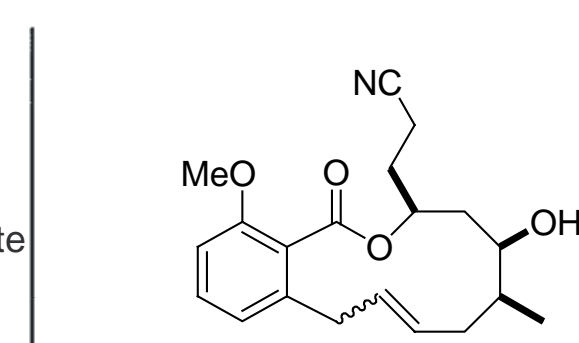

41

莺

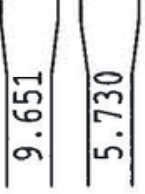

Z-isomer

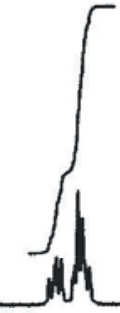

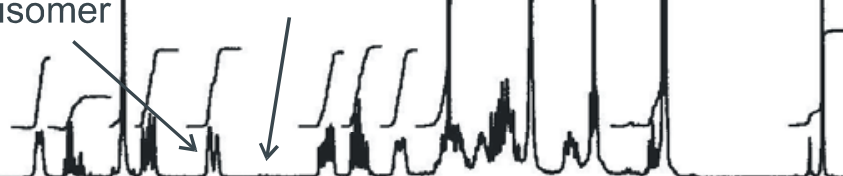
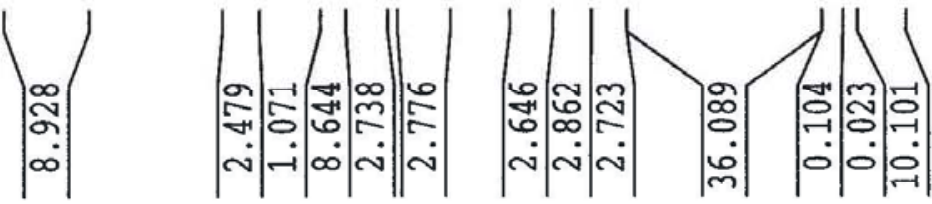


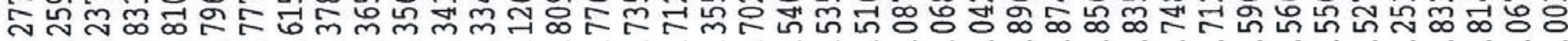
r. $\mathrm{H}_{1}^{L}$

\section{HPLC results (Silica gel 60):}

Gradient ethyl acetate in hexanes

$0 \%$ ethyl acetate to $30 \%$ ethyl acetate

(30 minutes)

Peak 1 (28.4 min): $96.4 \%$

Peak 2 (32.9 min): $3.6 \%$

NMR integration results:

$E: Z 26: 1$

Ratio determined based on NMR and HPLC

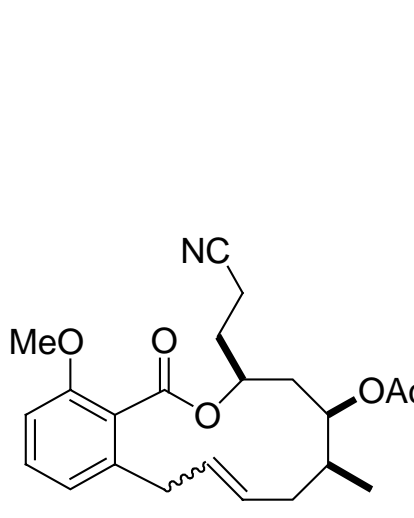

42

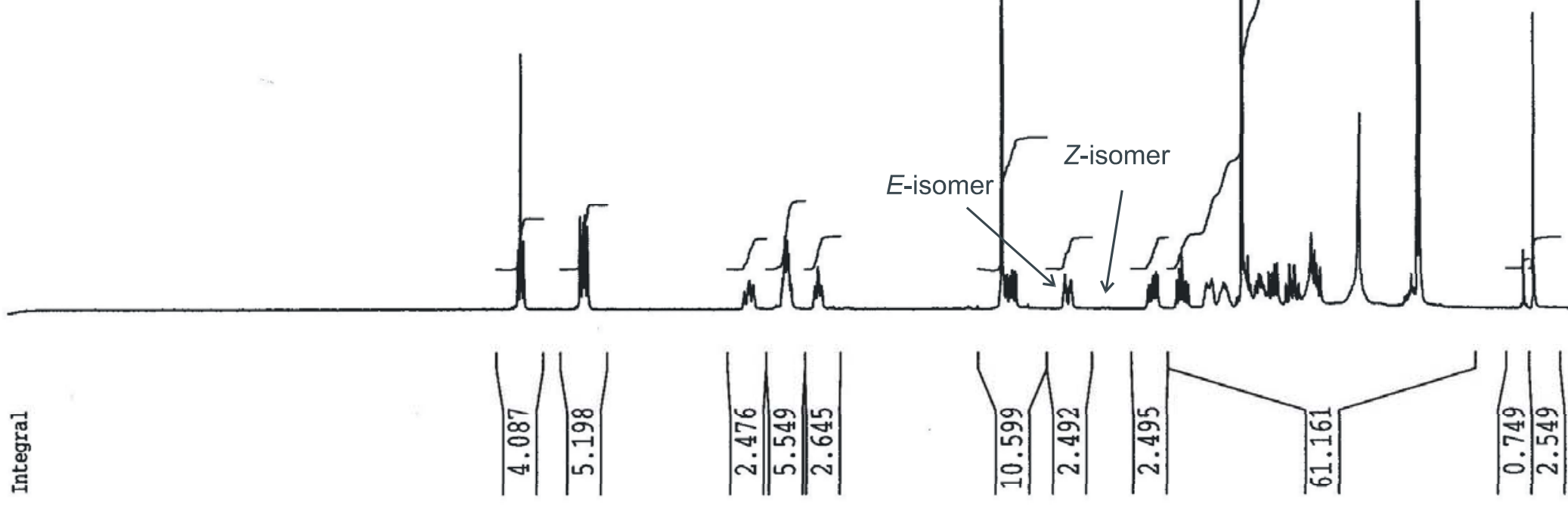

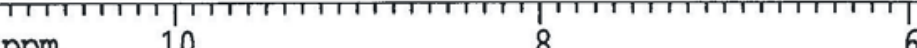

4

0 


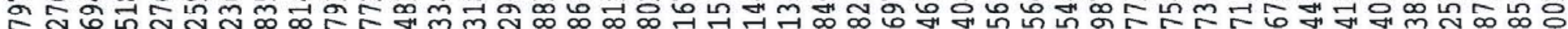
0

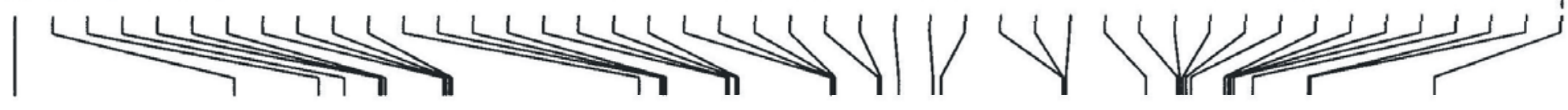

HPLC results (Silica gel 60):

Gradient ethyl acetate in hexanes $0 \%$ ethyl acetate to $30 \%$ ethyl acetate (30 minutes)

Peak 1 (31.6 min): 96.9\%

Peak 2 (32.8 $\mathrm{min}): 3.1 \%$

NMR integration results:

$E: Z 23: 1$

Ratio determined based on NMR

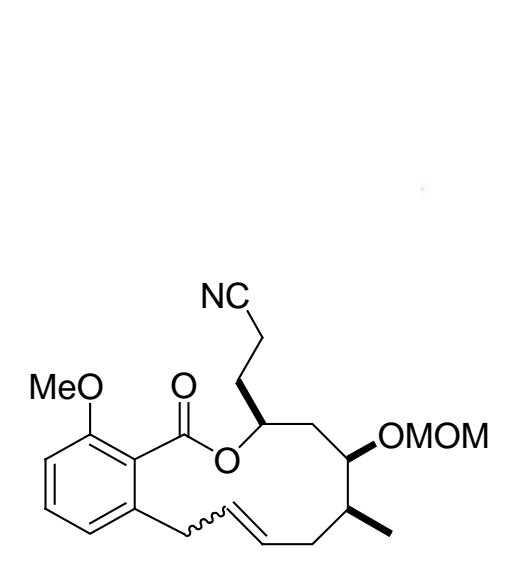

43

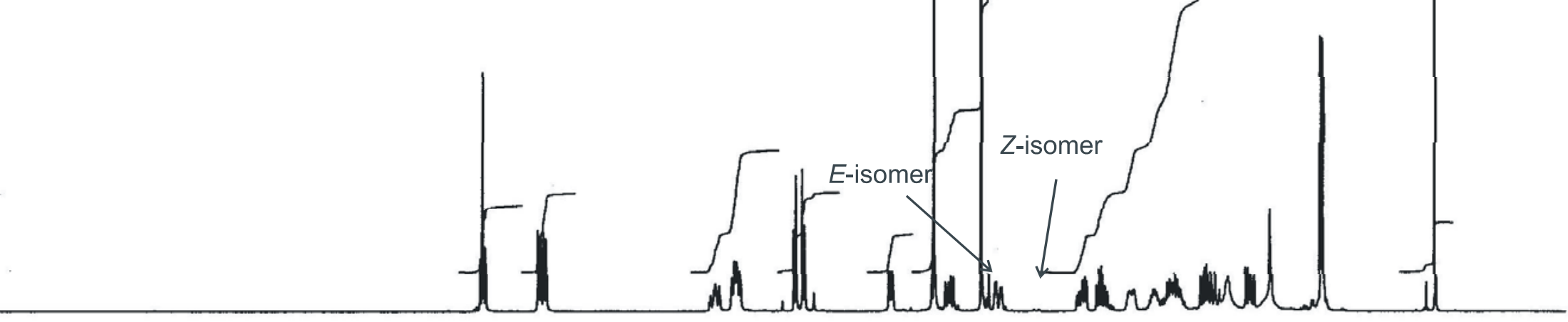

鸪
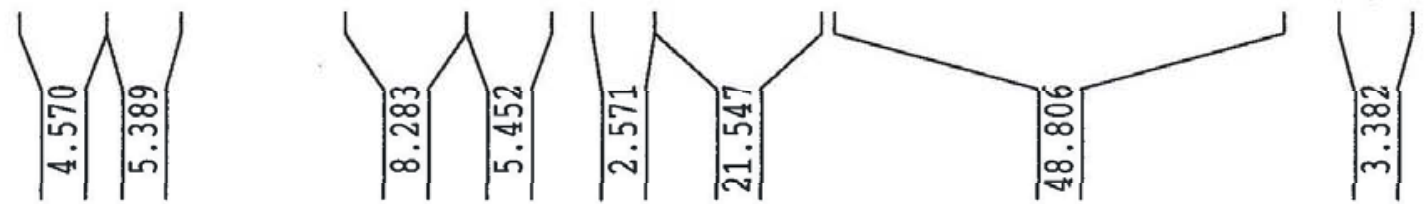


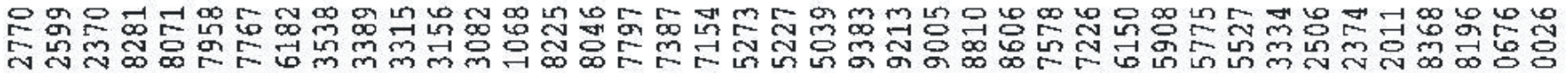
ب̂.

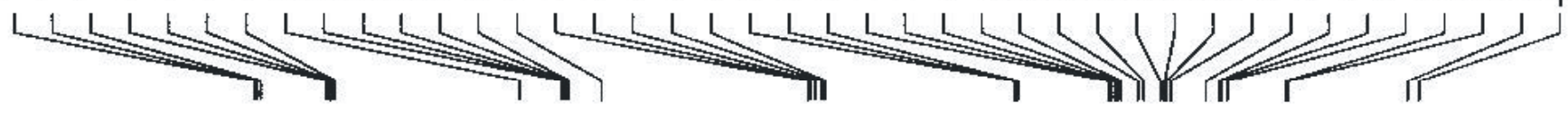

HPLC results (Silica gel 60):

Gradient ethyl acetate in hexanes

$0 \%$ ethyl acetate to $30 \%$ ethyl acetate

(30 minutes)

Peak 1 (29.7 min): 95.4\%

Peak 2 (30.9 min): 4.6\%

NMR integration results:

$E: Z 22: 1$

Ratio determined based on NMR and HPLC

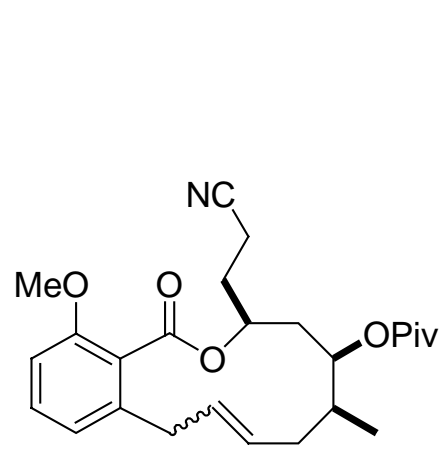

44

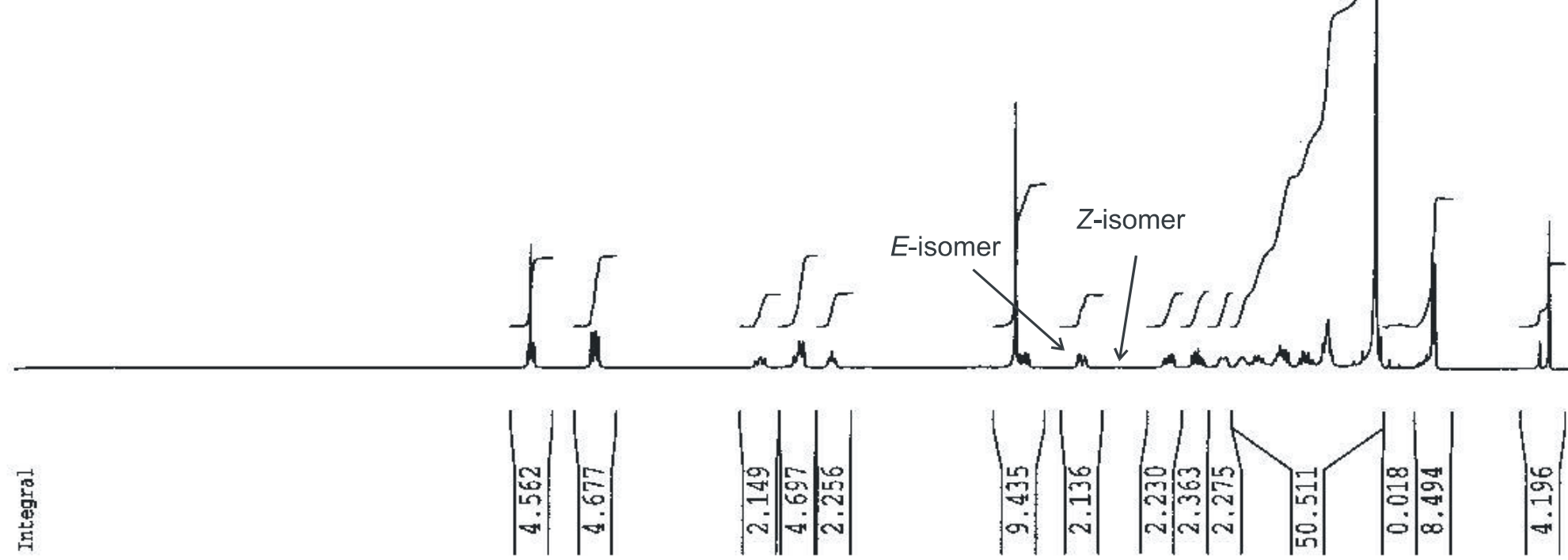

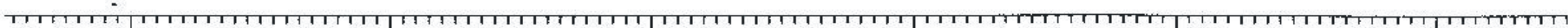




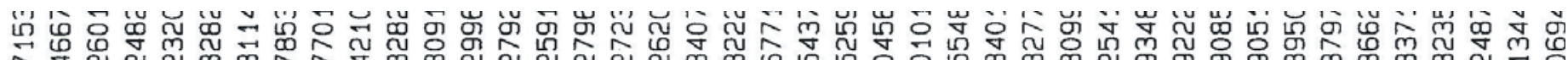

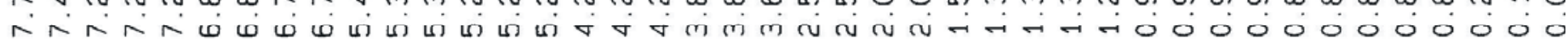
$\underbrace{L L L L L L L L L L L L L L L L L L L L L L L L L L U}_{7 \rightarrow 1}$

HPLC results (Silica gel 60):

Gradient ethyl acetate in hexanes $0 \%$ ethyl acetate to $20 \%$ ethyl acetate (30 minutes)

Peak 1 (37.3 $\mathrm{min}): 94.9 \%$

Peak 2 (39.5 min): 5.1\%

NMR integration results:

$E: Z 16: 1$

Ratio determined based on NMR

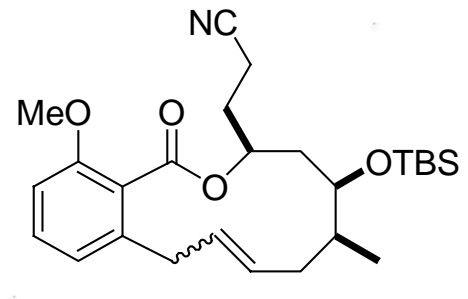

45
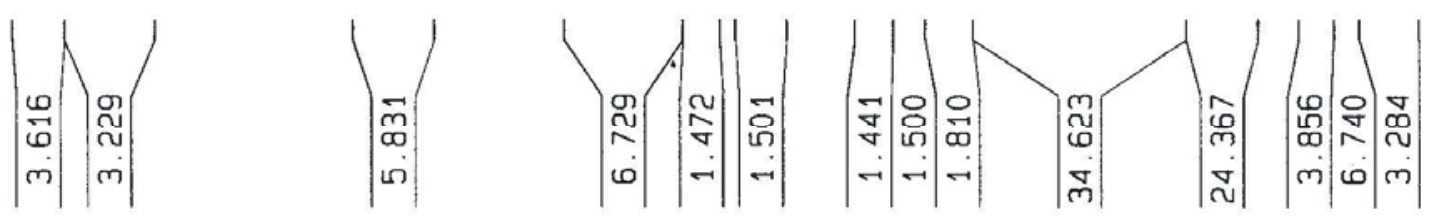

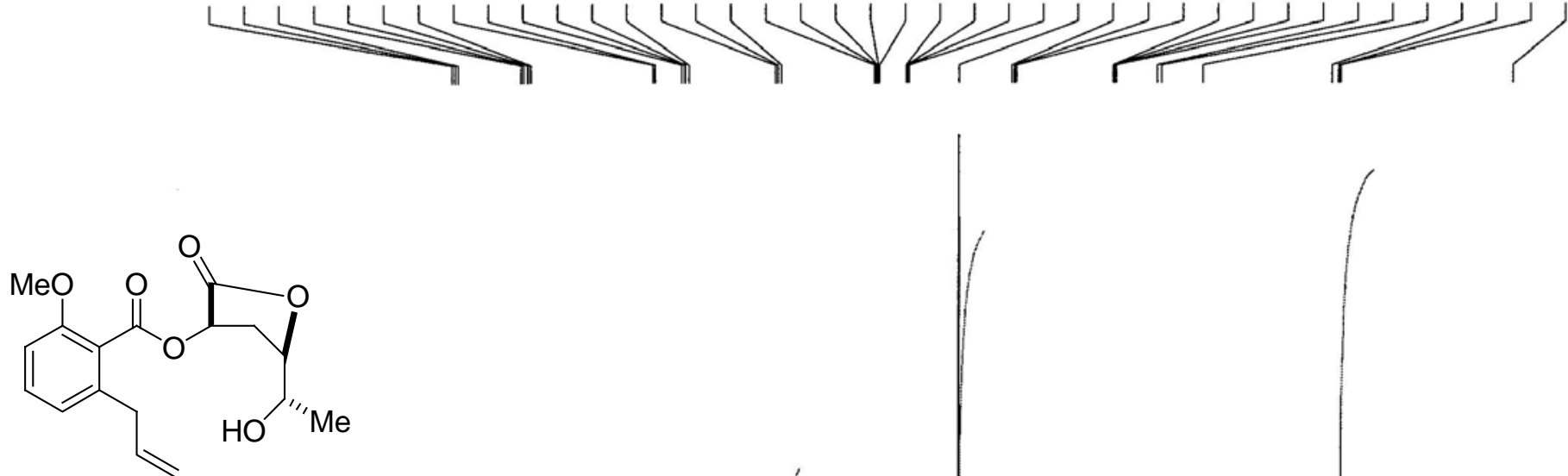

46

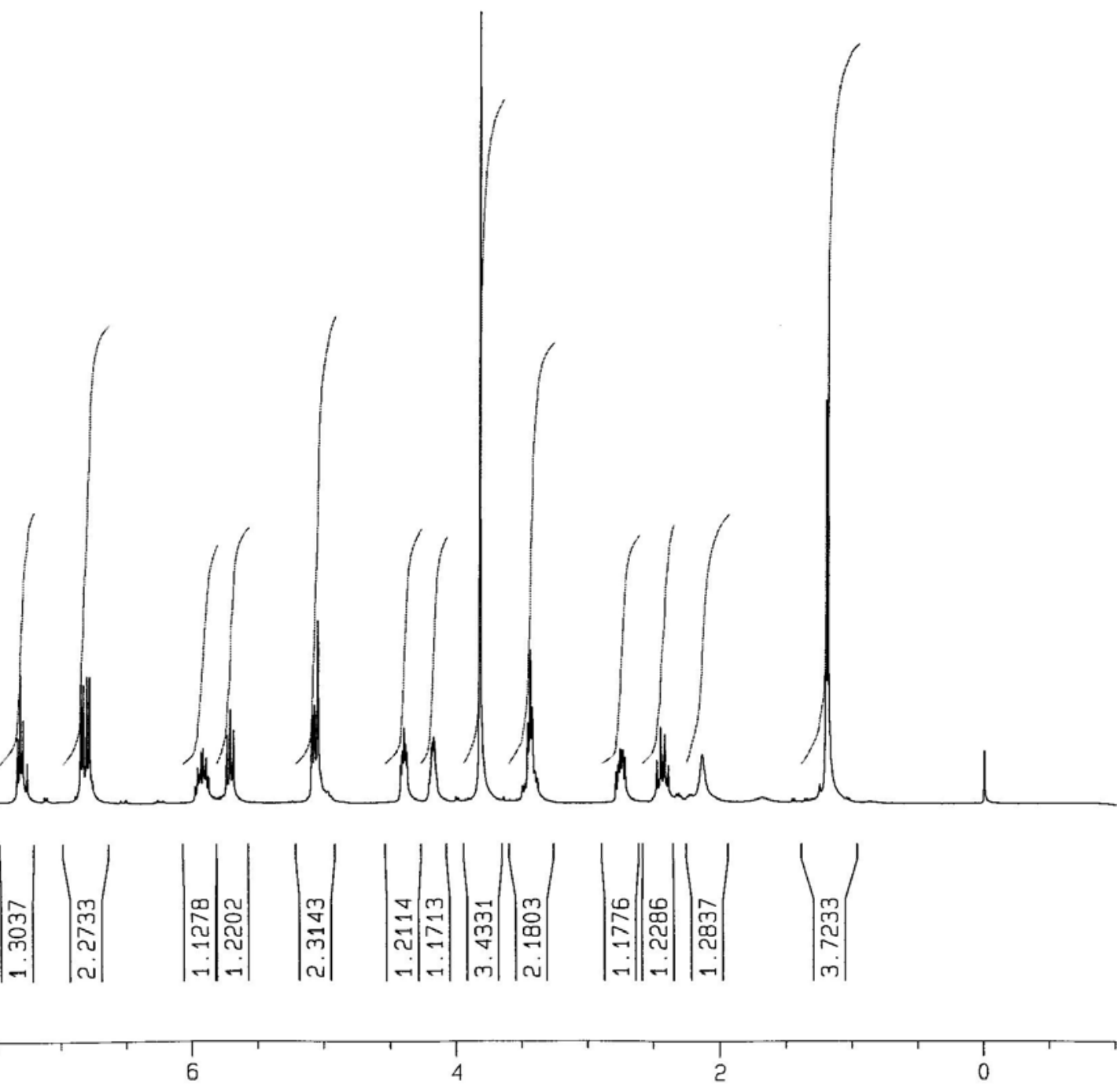




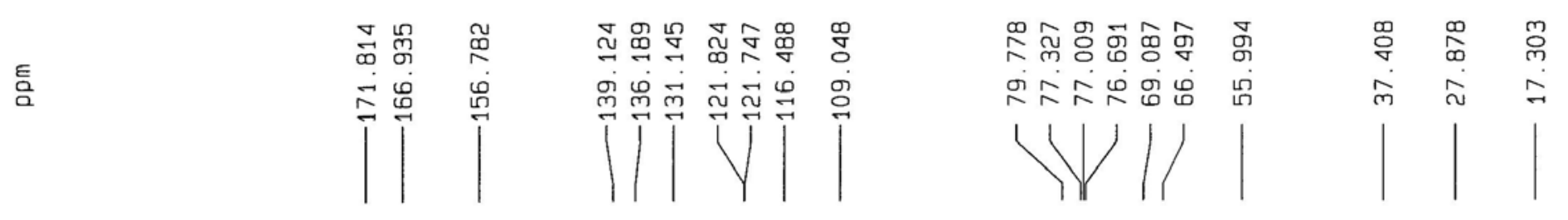

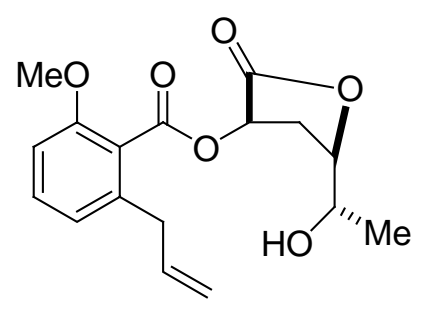

46

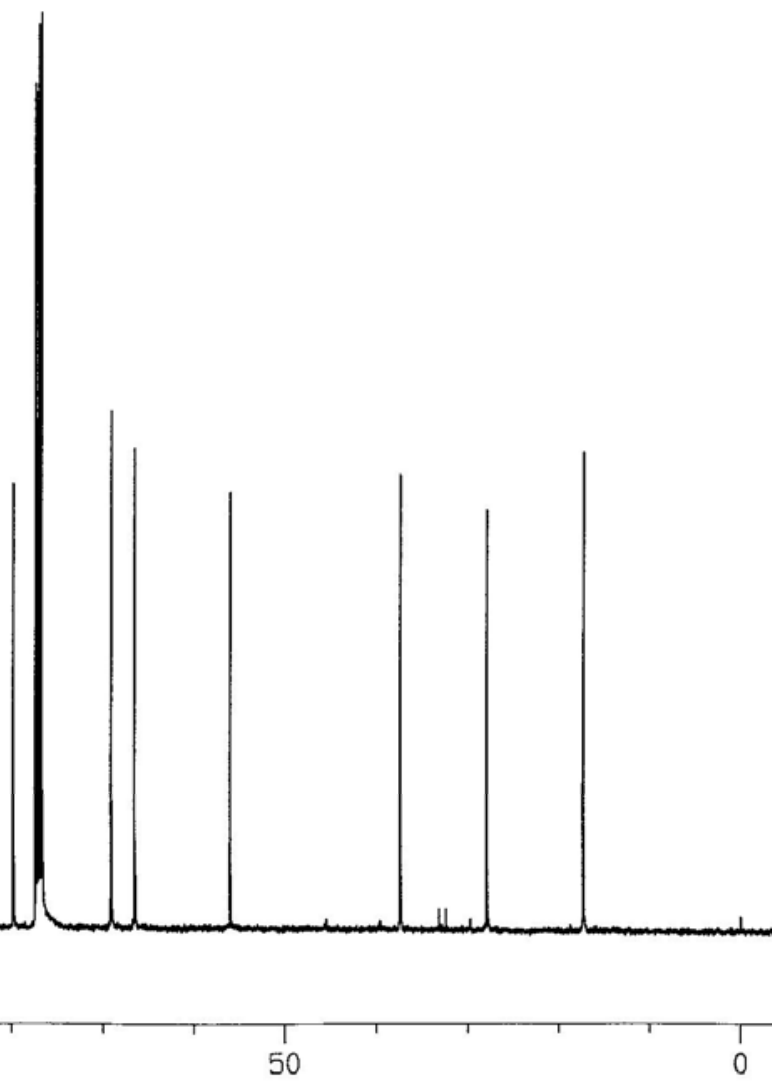




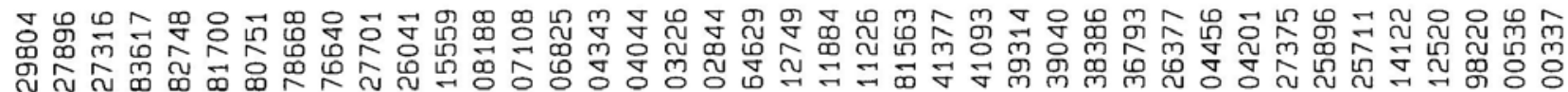

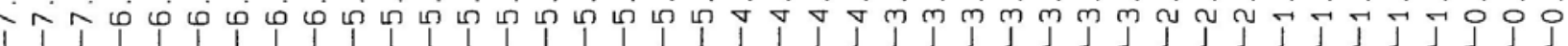
$\rightarrow$
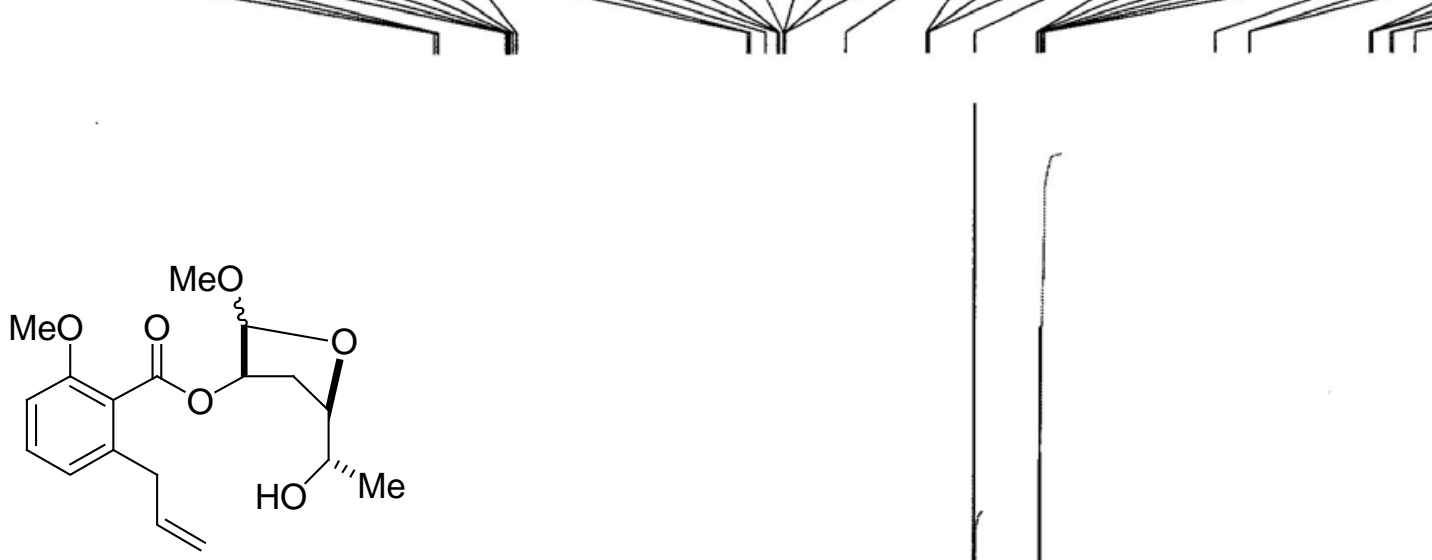

47

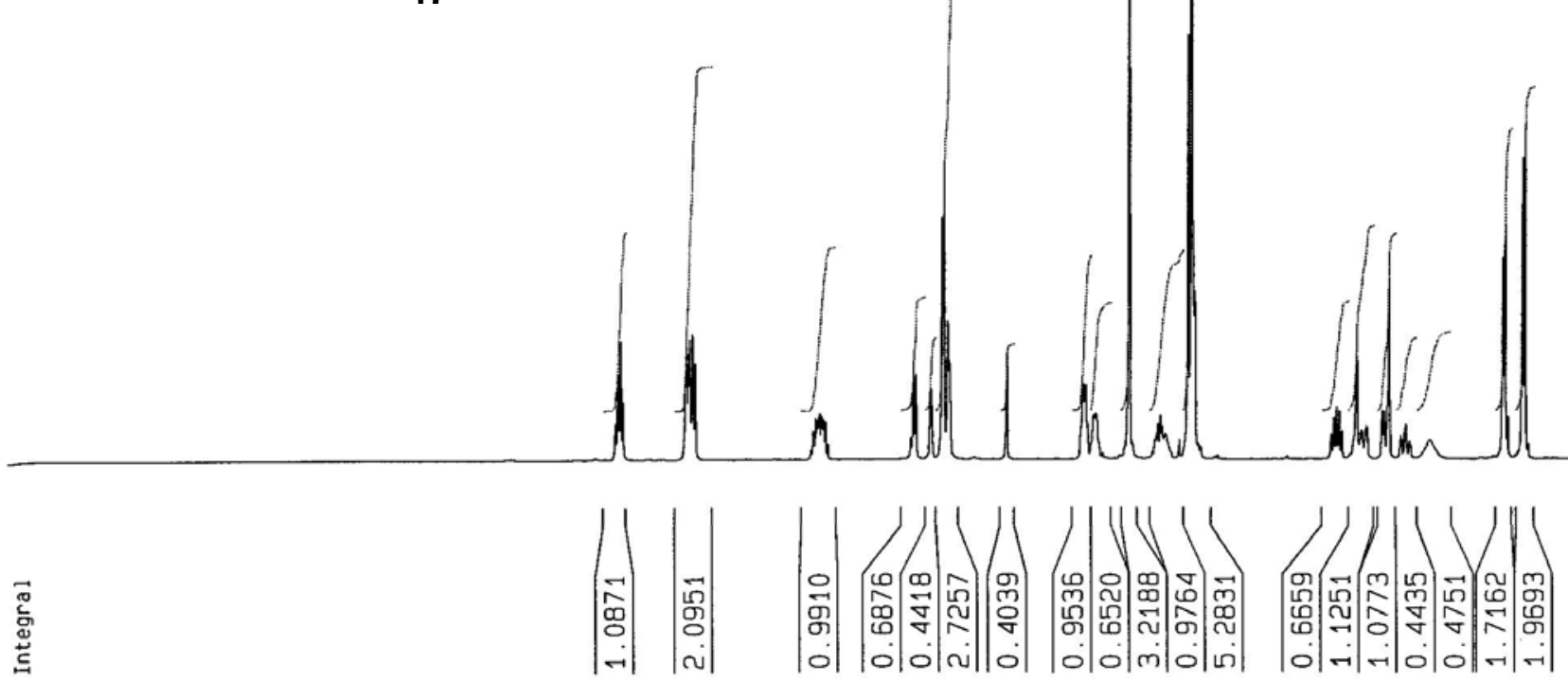




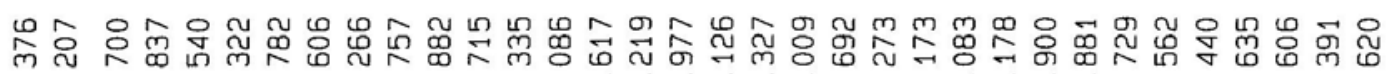
ம்

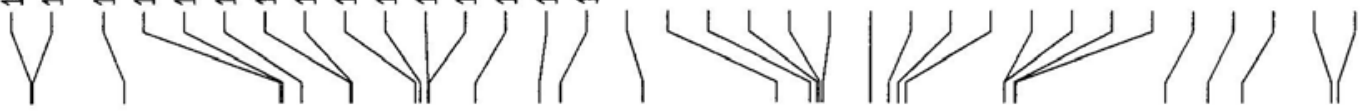

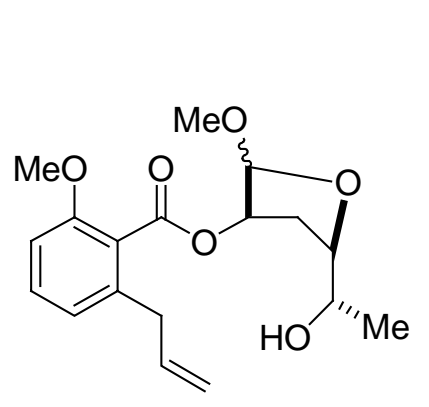

47 

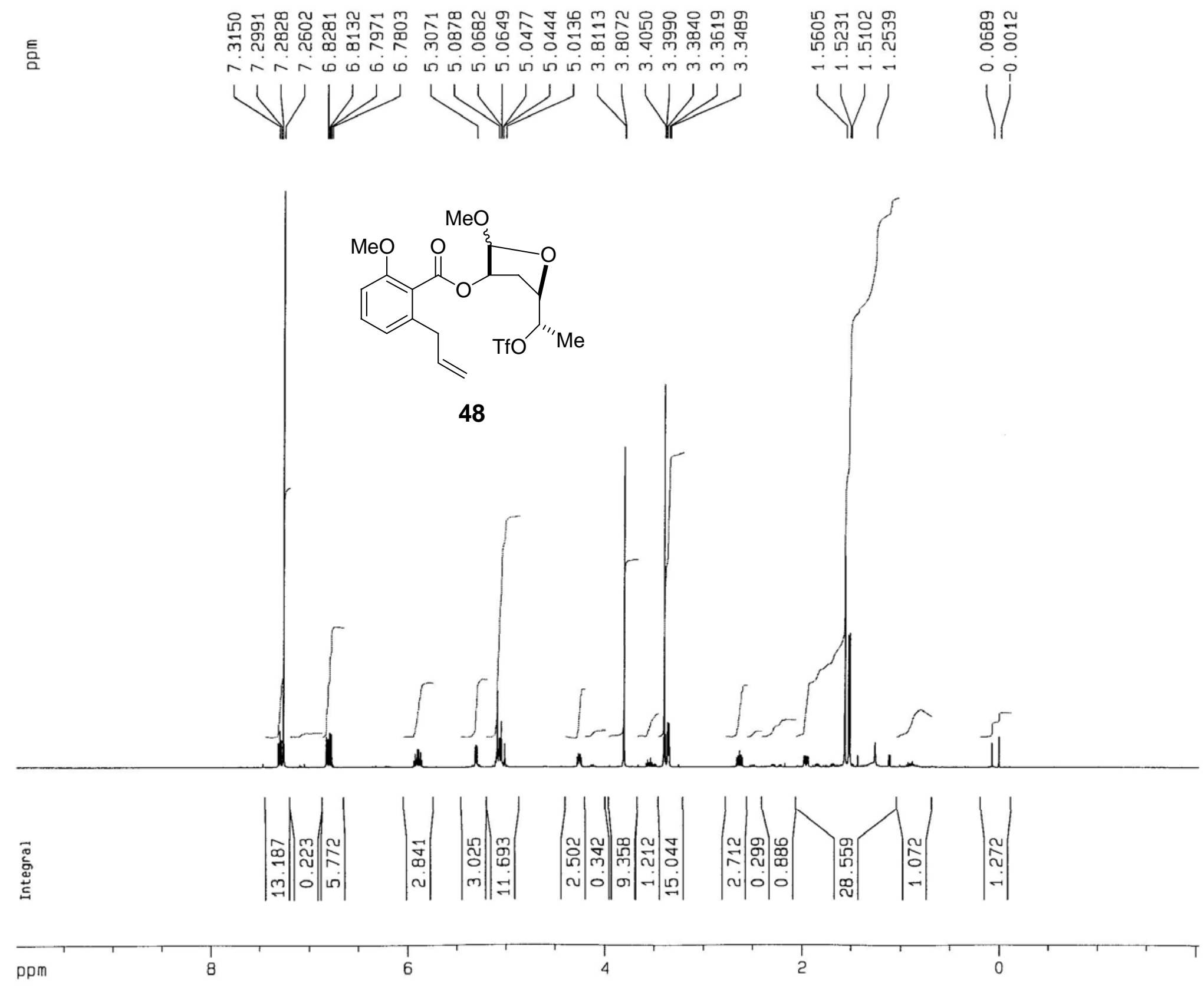


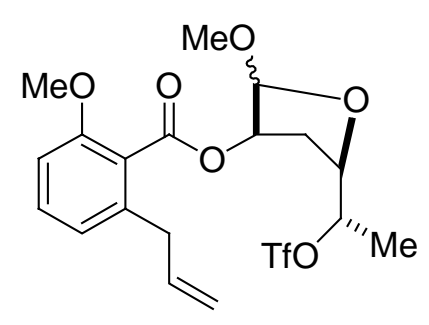

48

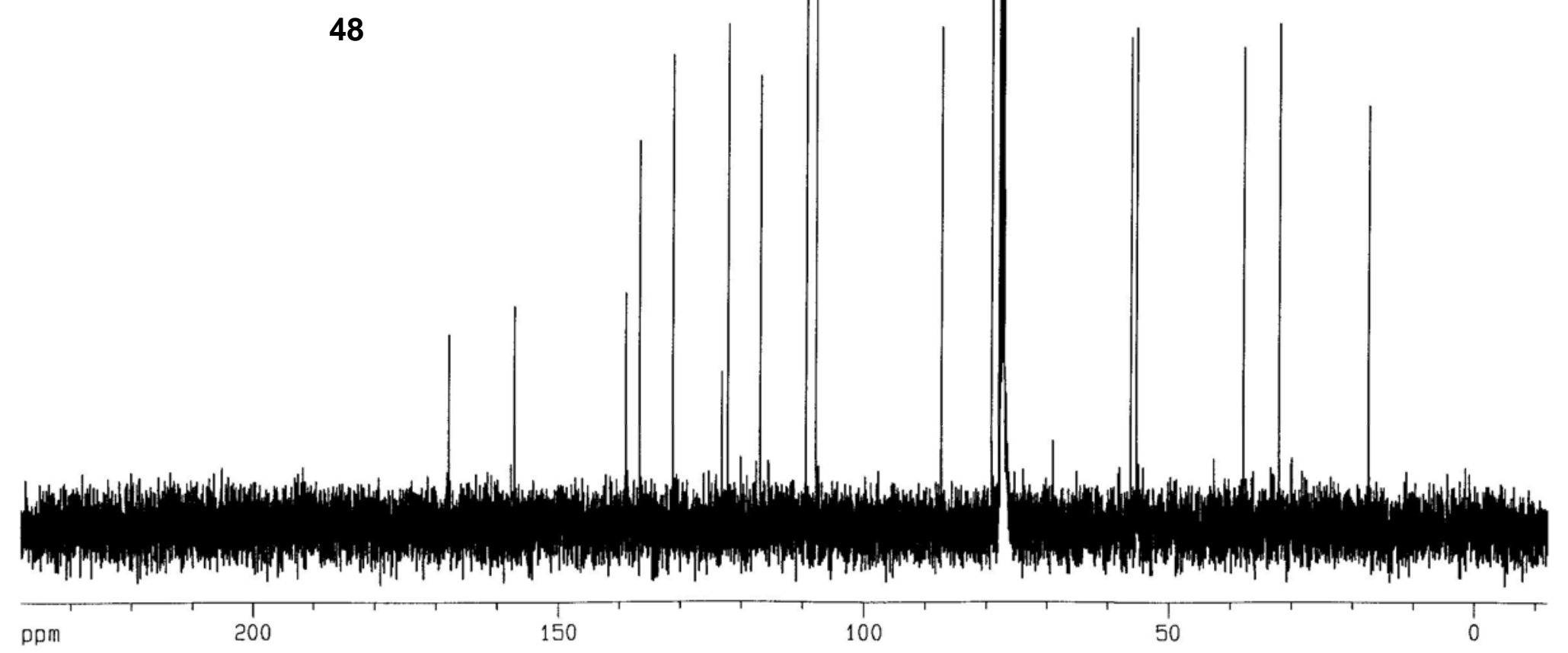




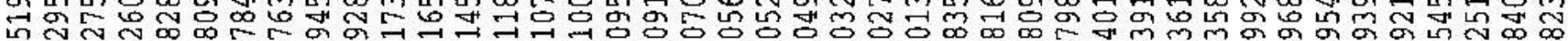
rí
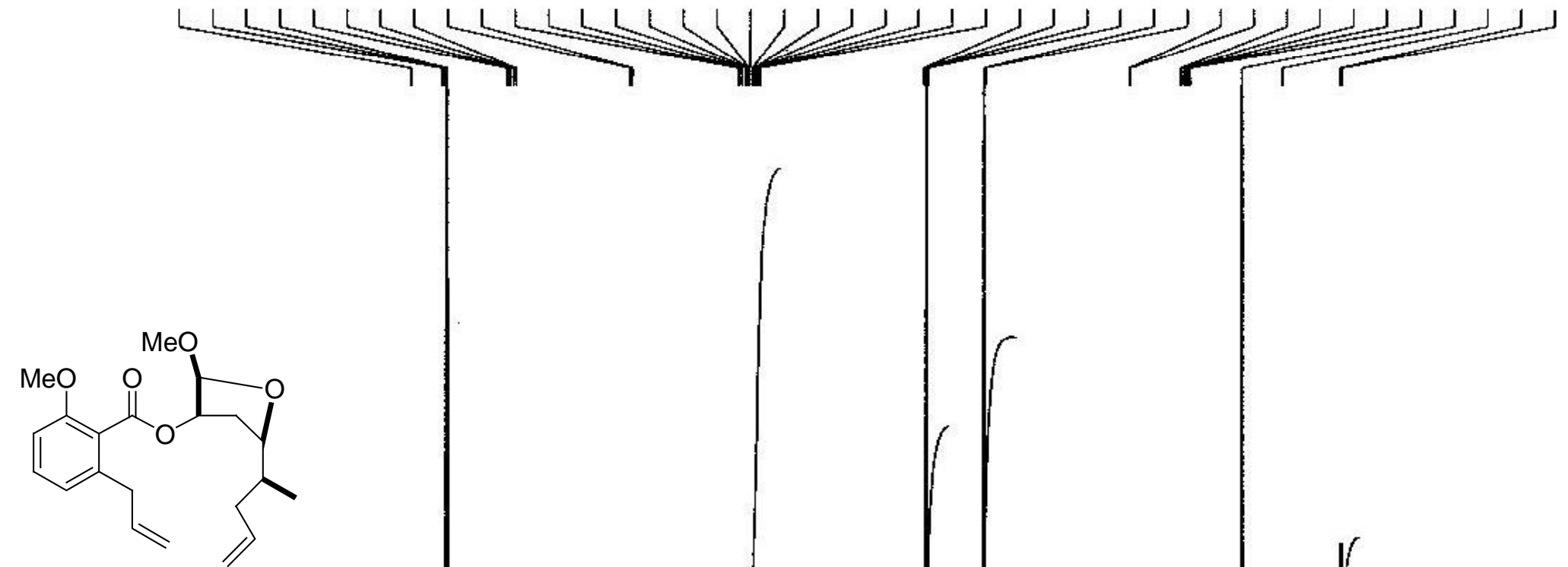

49-alpha anomer

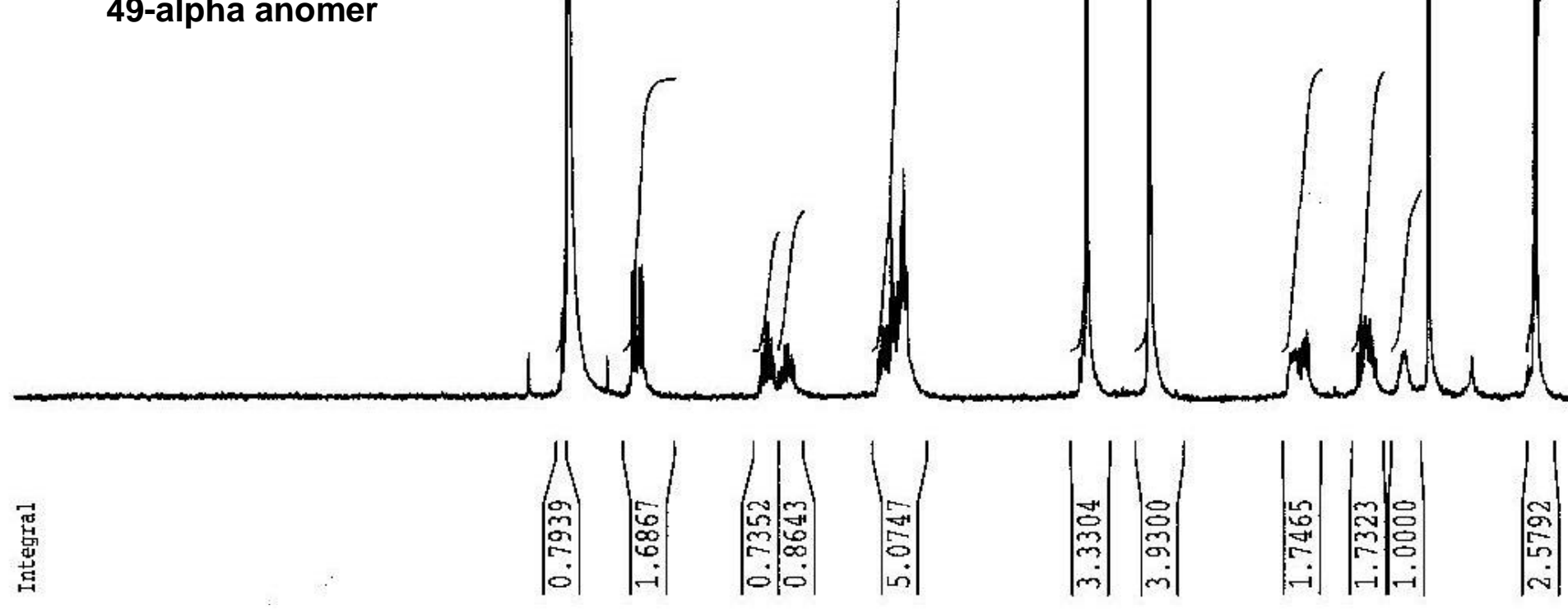




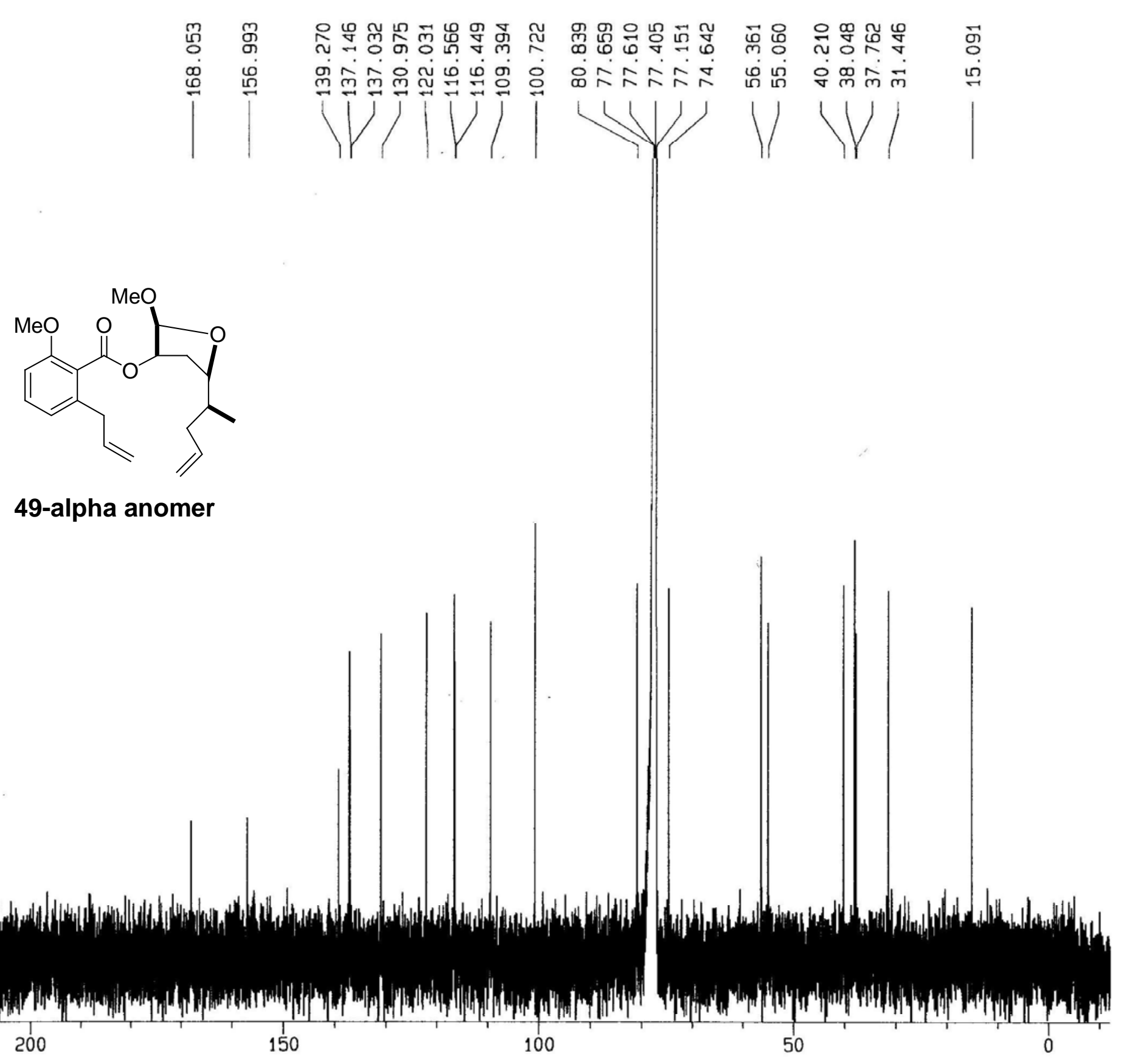



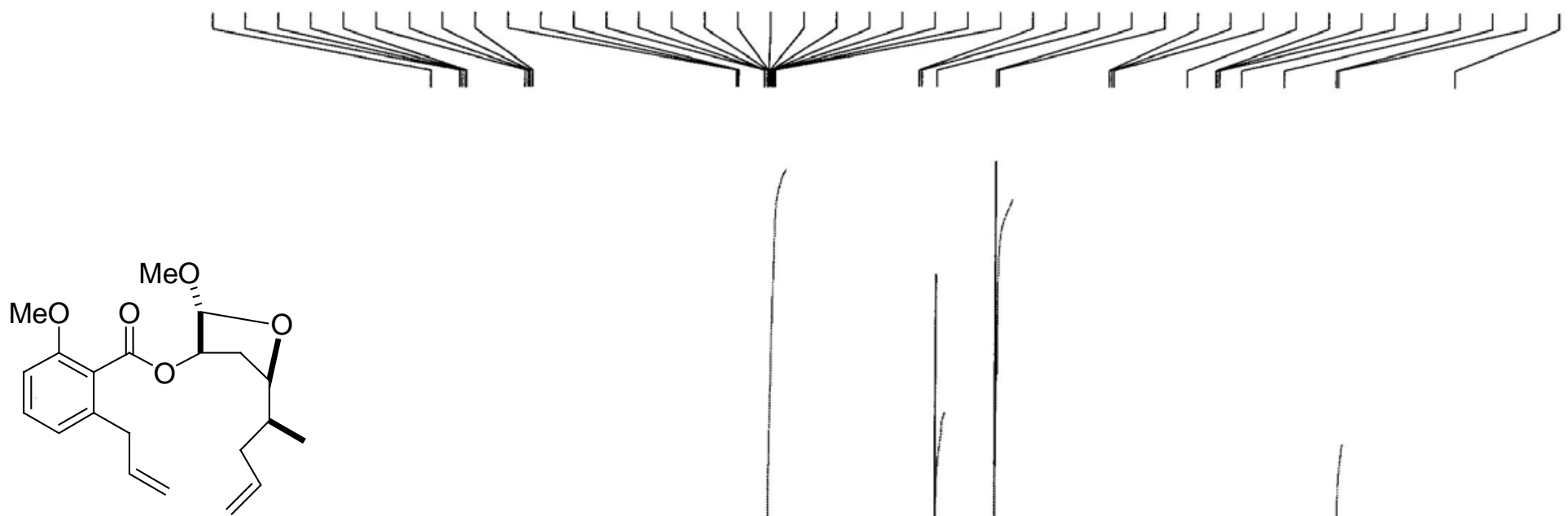

49-beta anomer

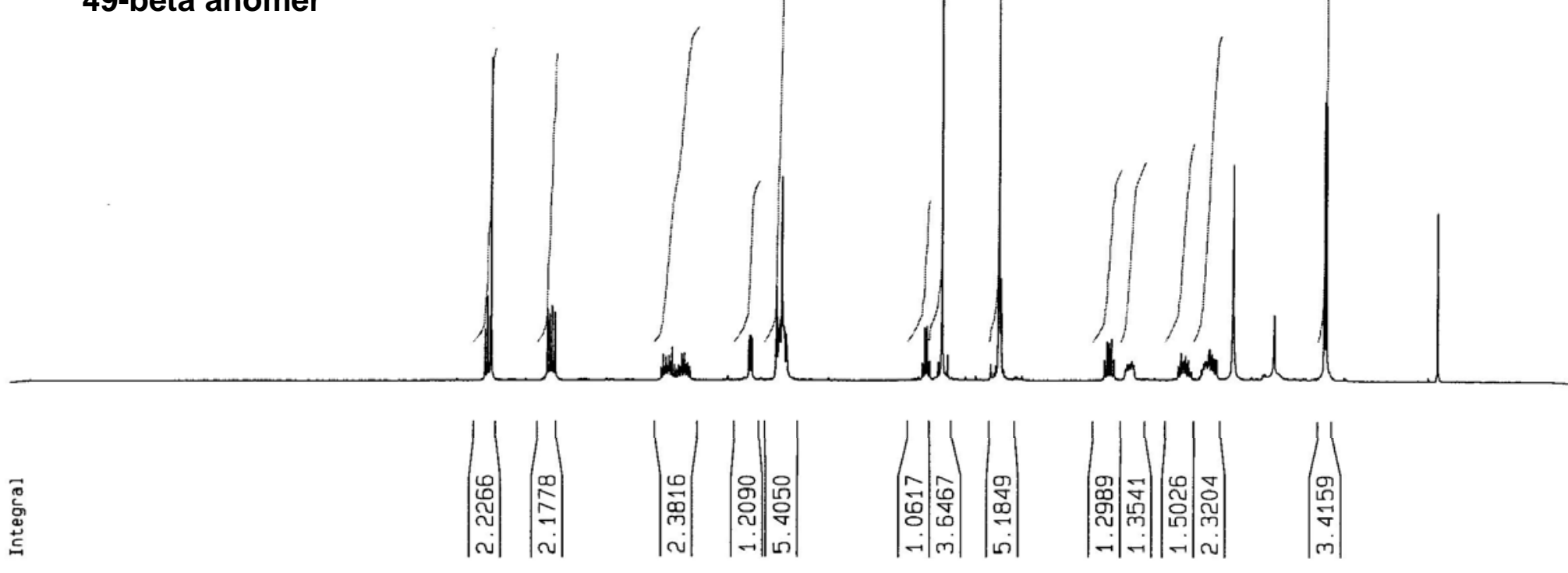

5 


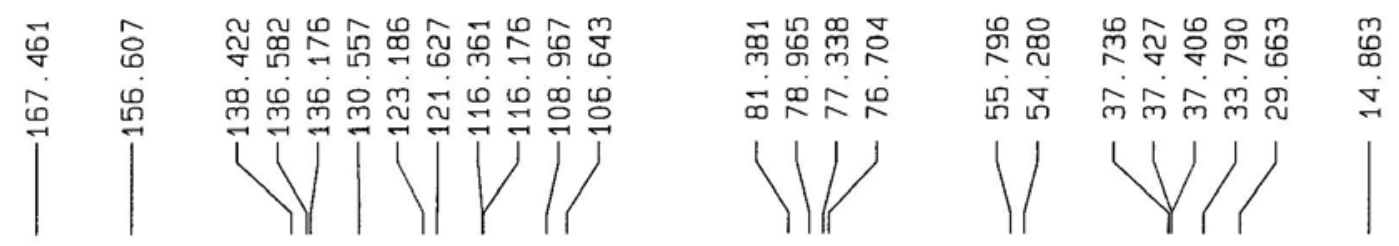

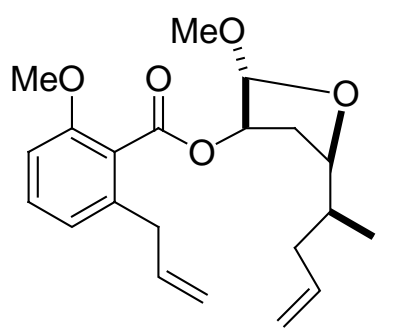

49-beta anomer

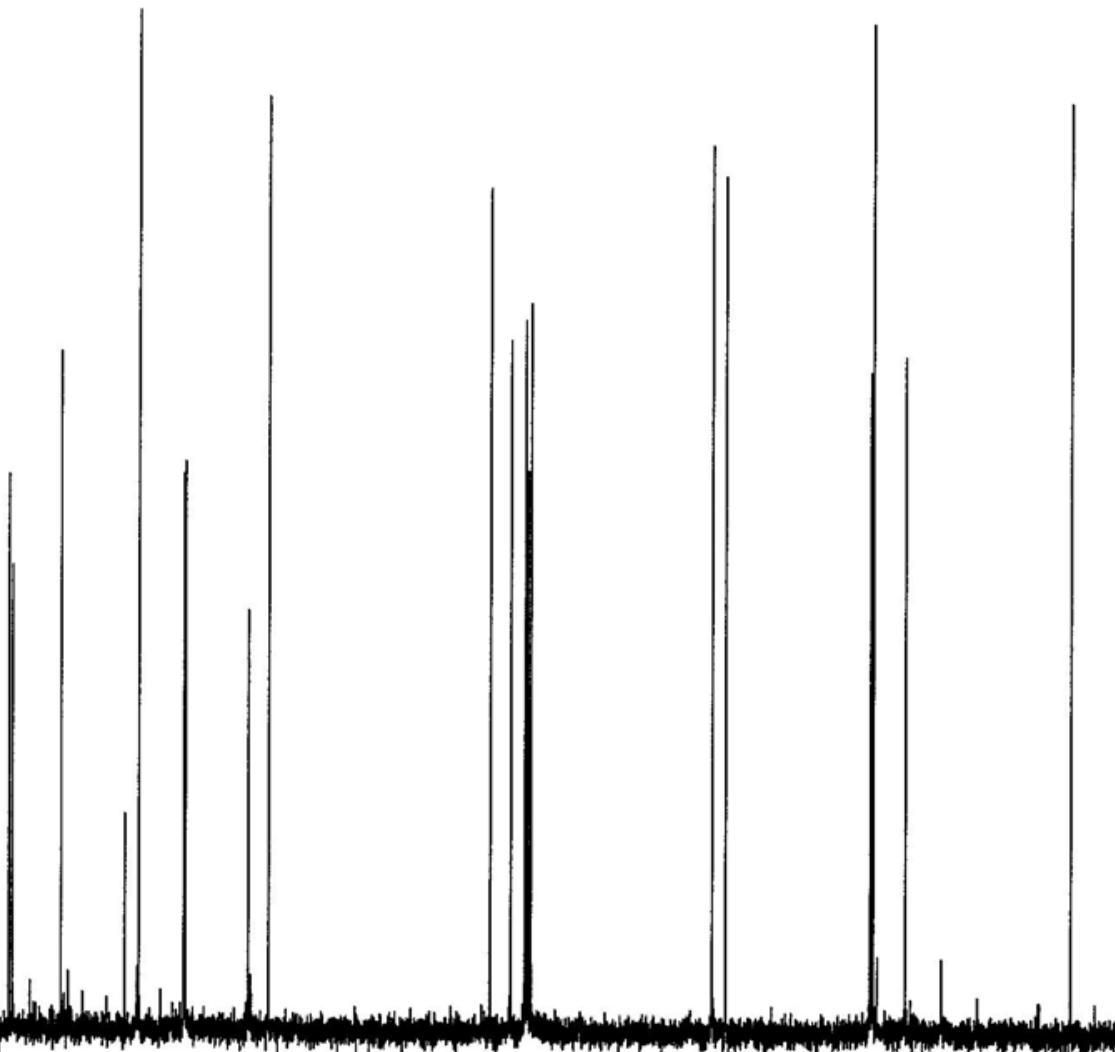




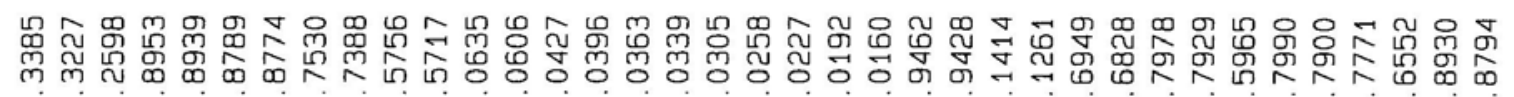

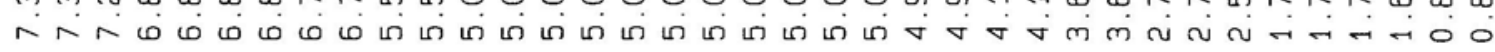
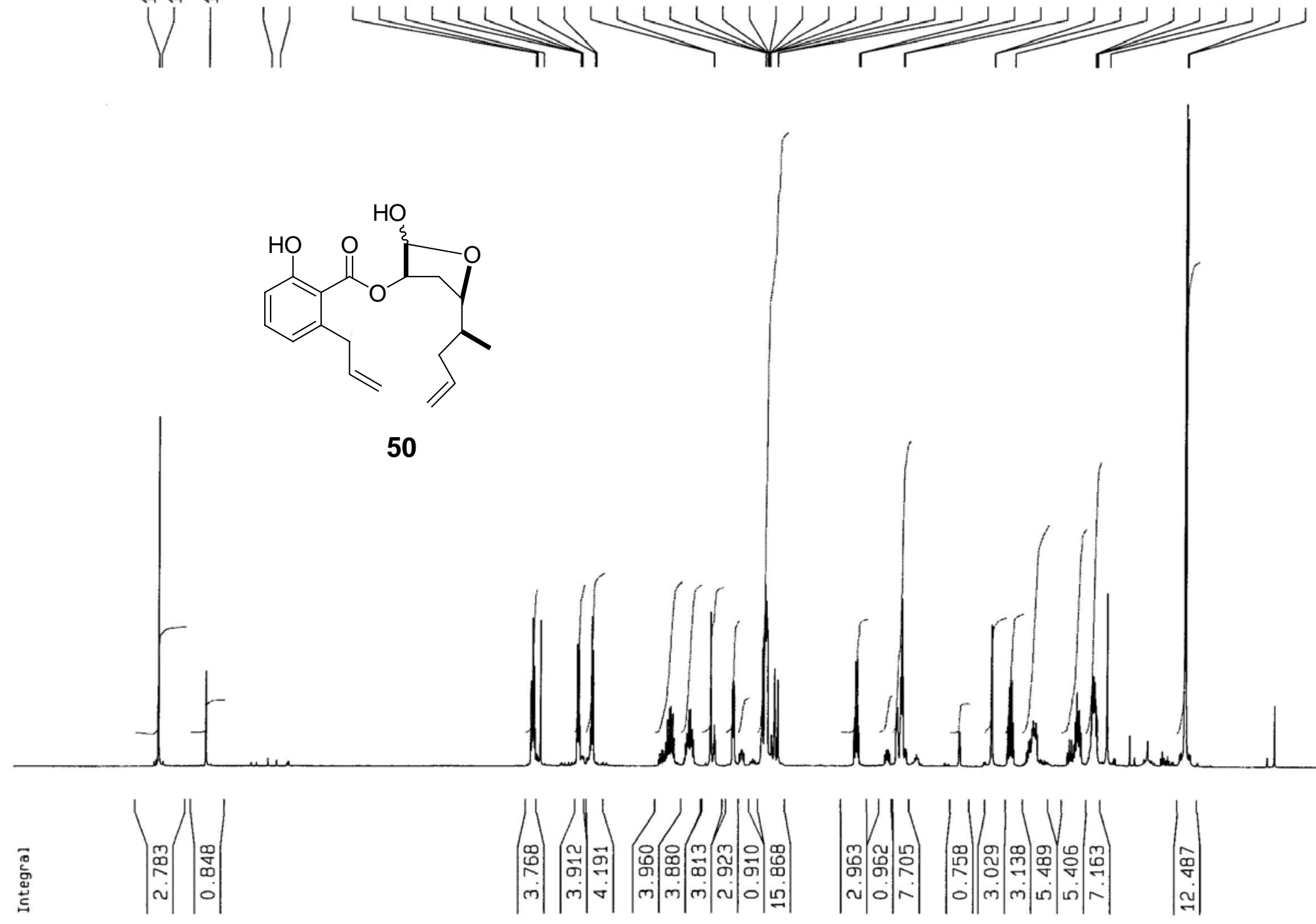

50

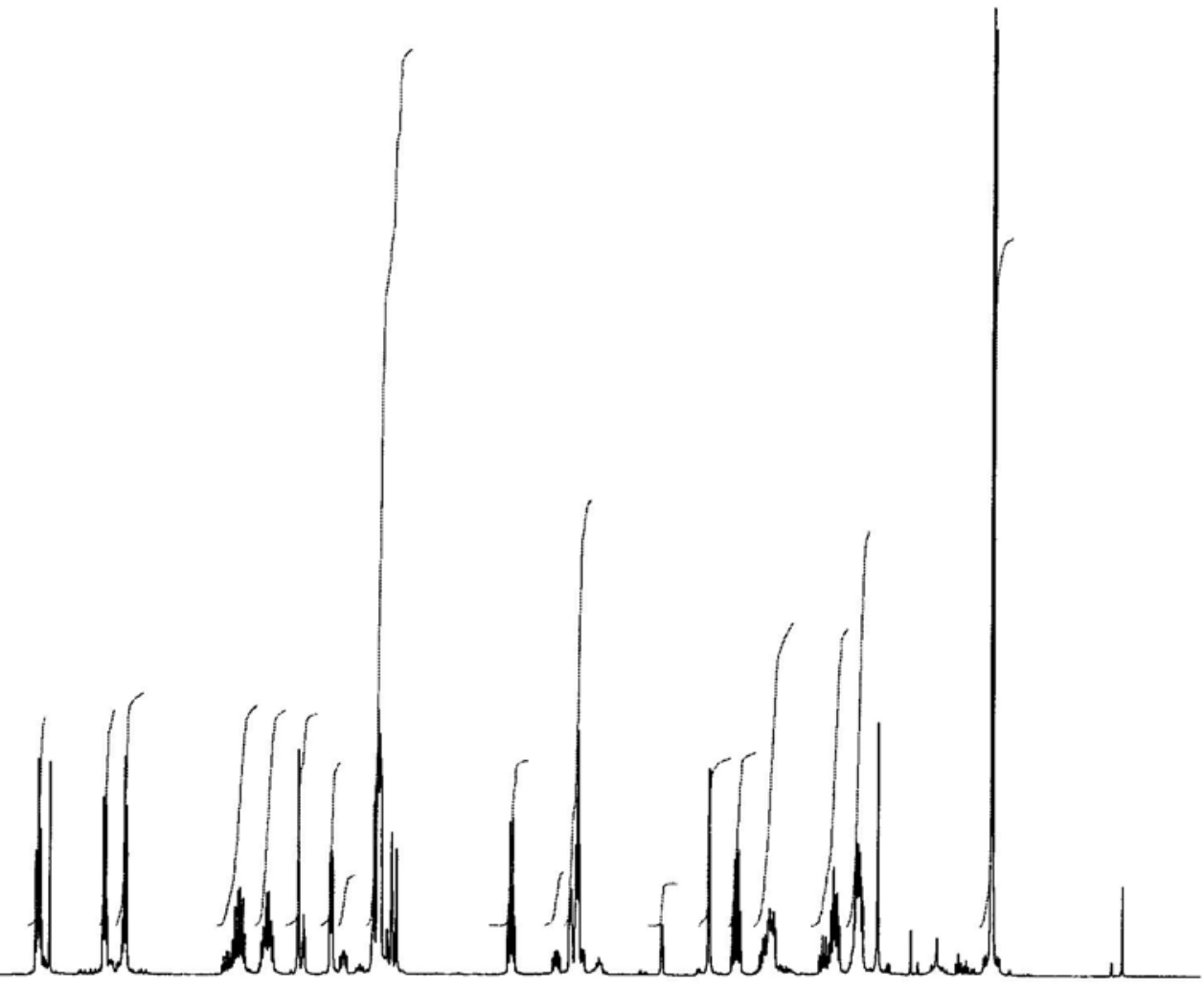




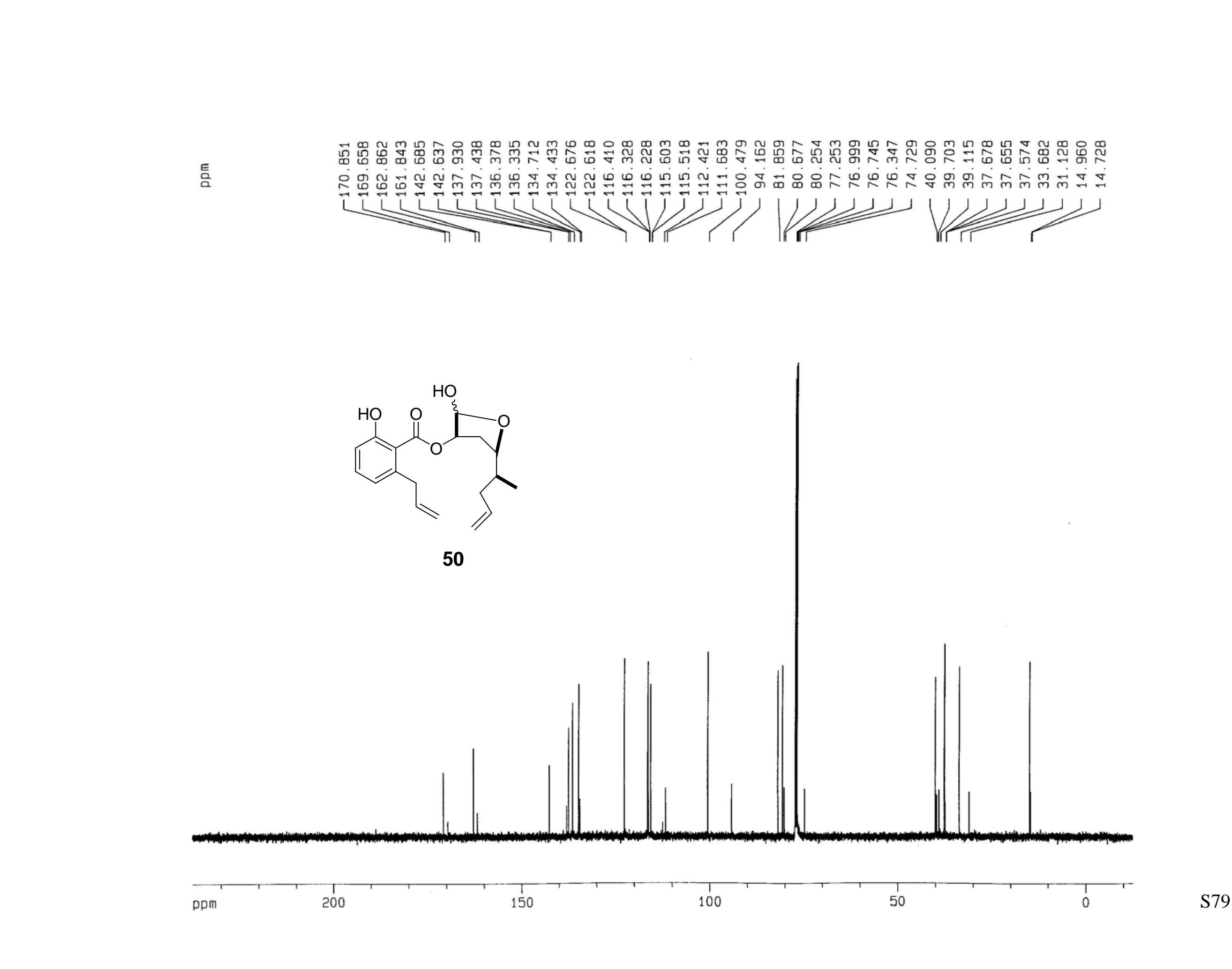




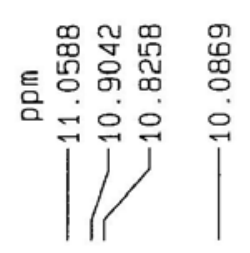

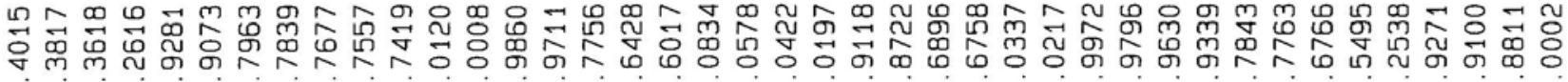
ヘ ヘ ヘ ம
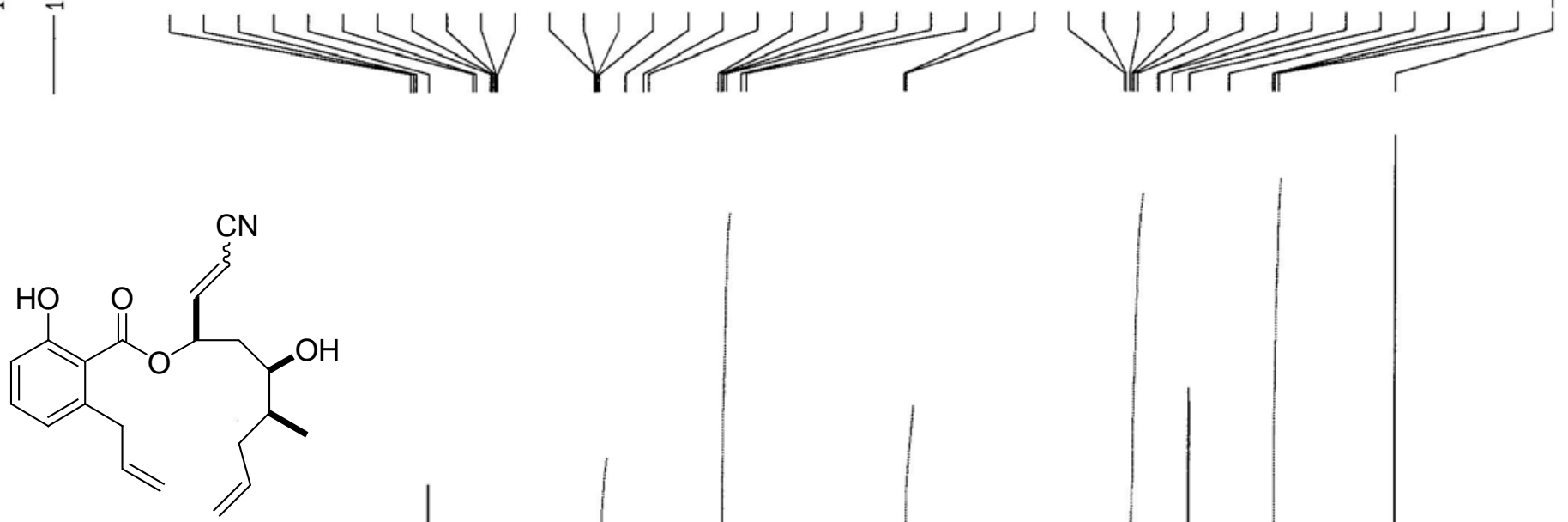

51
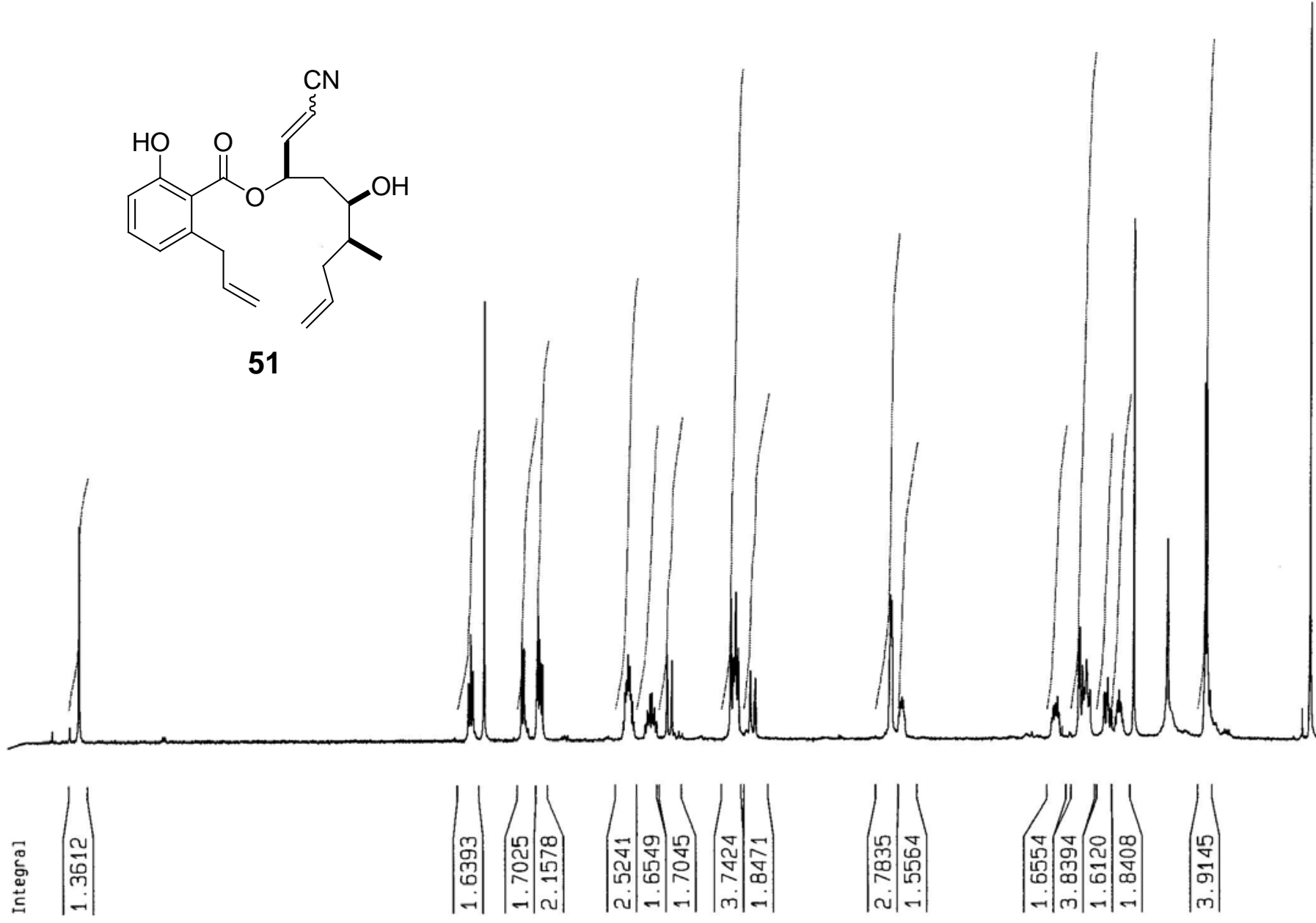
喜
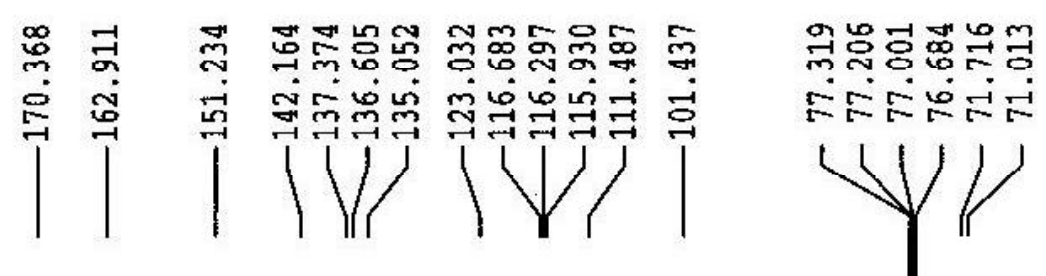

국్ㅀㅇㅊㅇㅇㅇㅇㅇㅛ

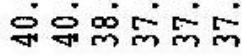

U

亲<smiles>C=CCc1cccc(O)c1C(=O)OC(C=CC#N)CC(O)C(C)CC=C</smiles>

51

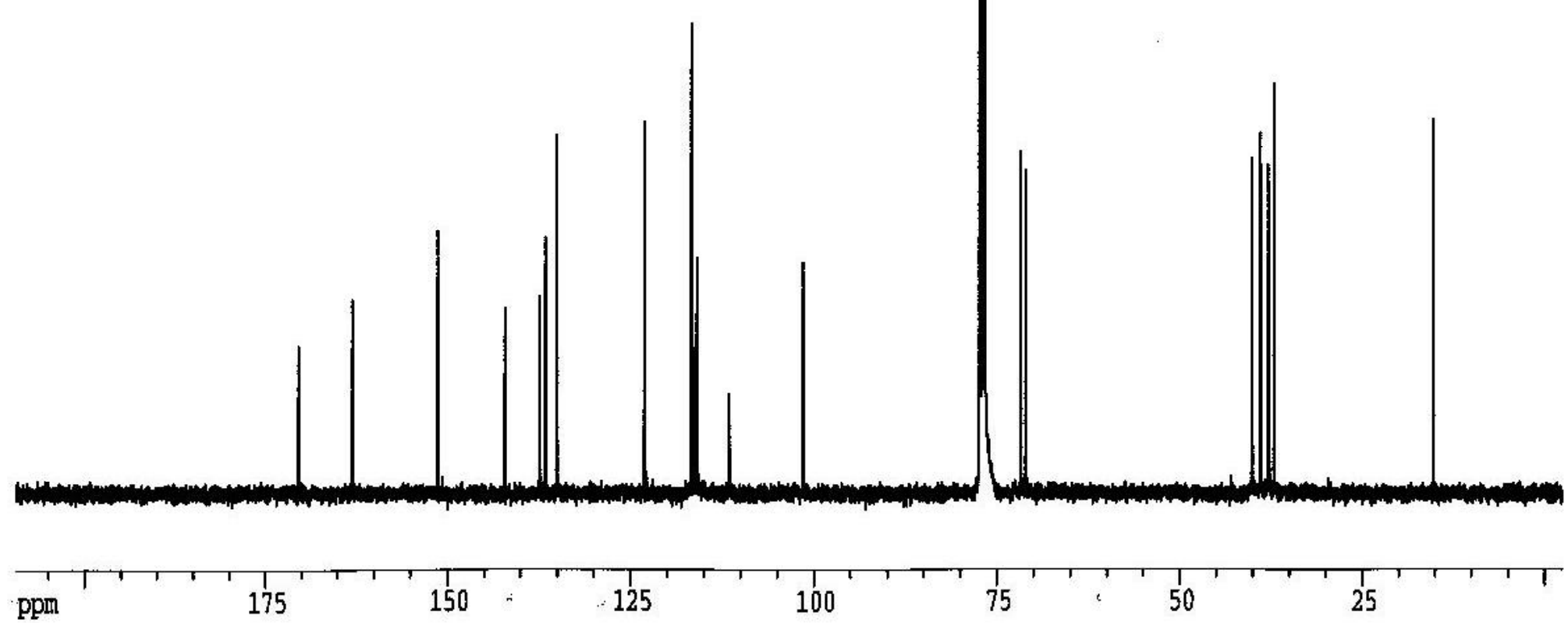


言 $\stackrel{0}{0}$

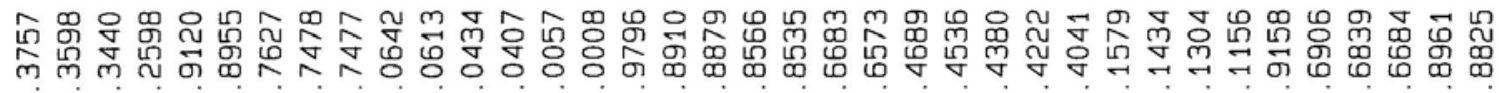
ヘヘ
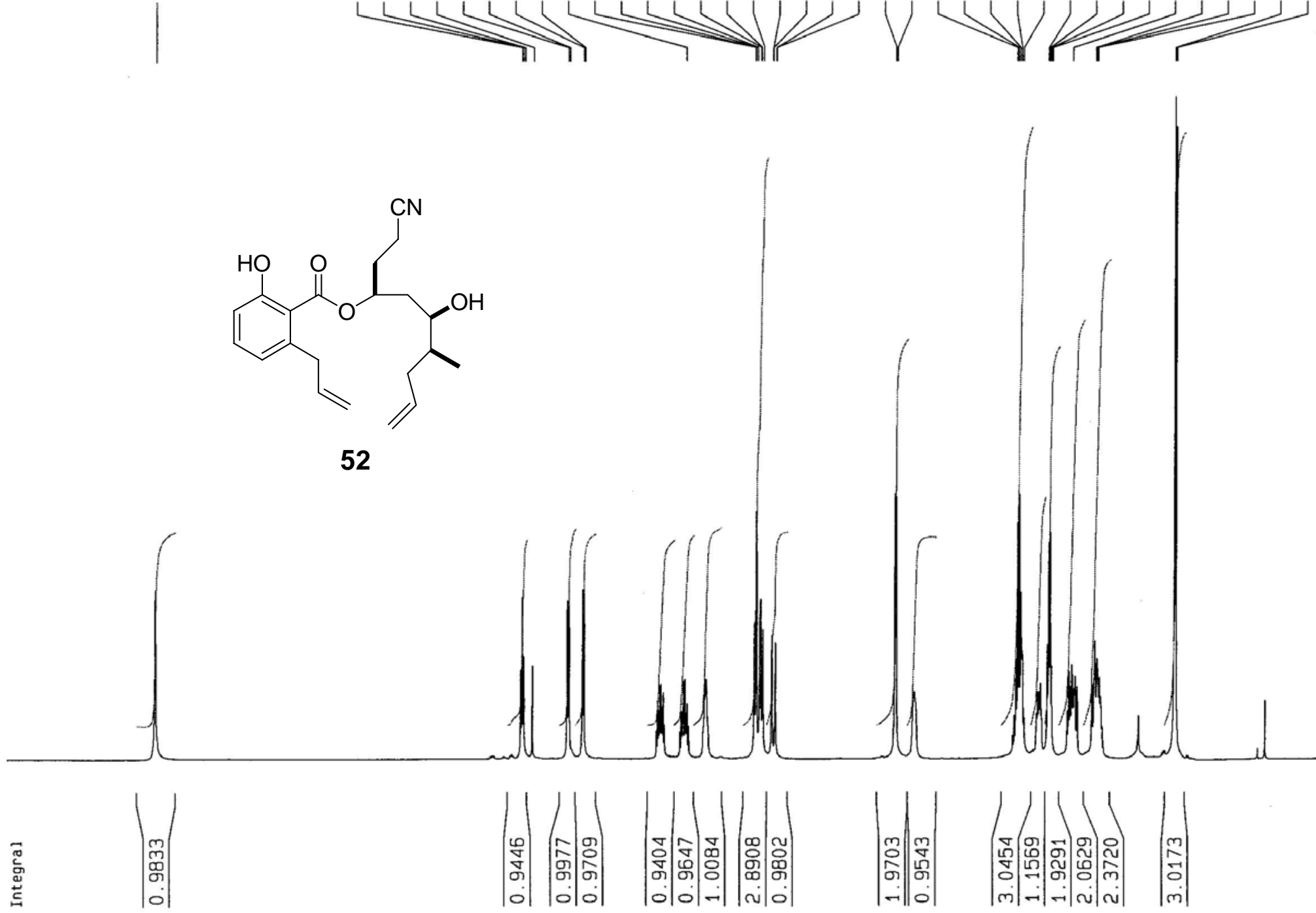


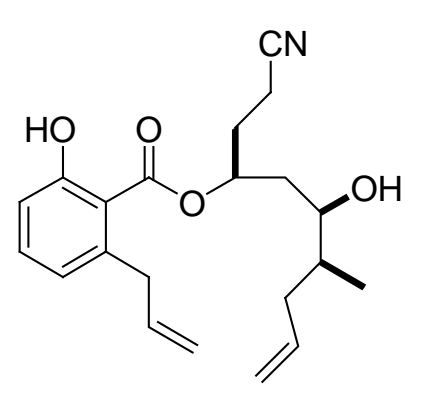

52

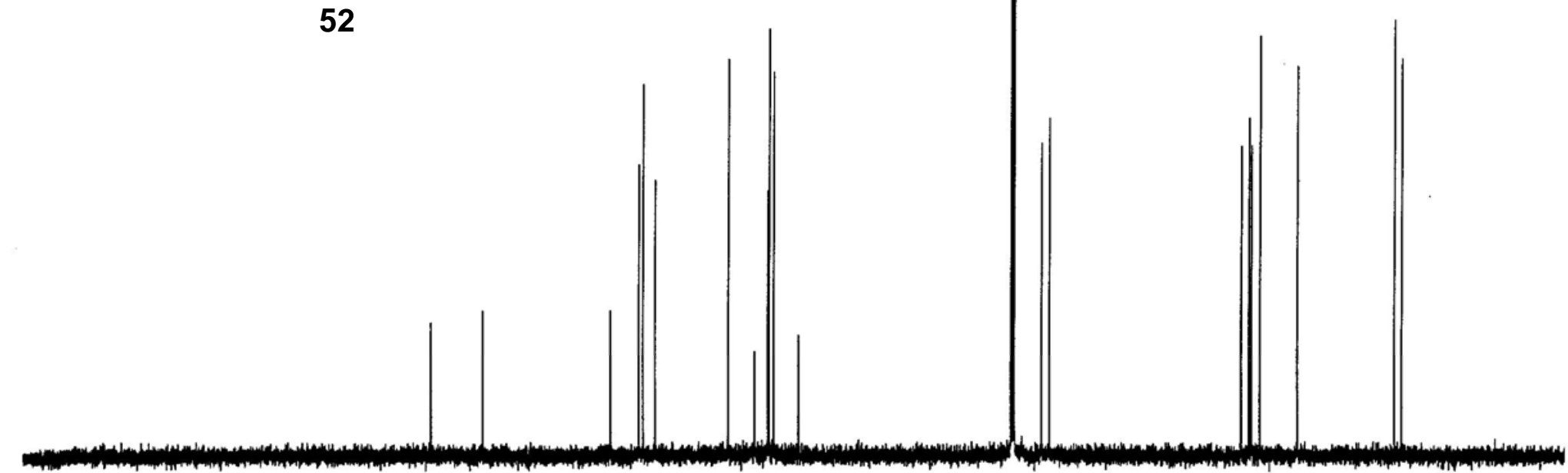



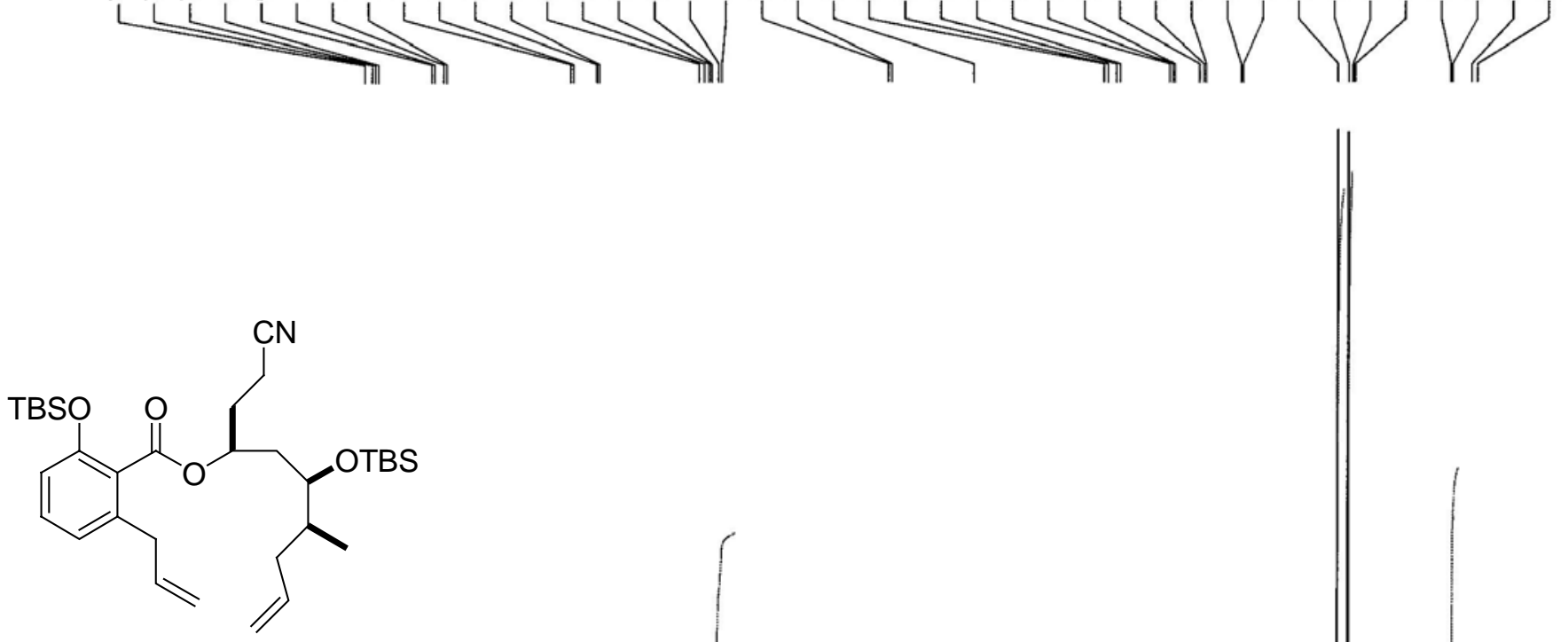

53

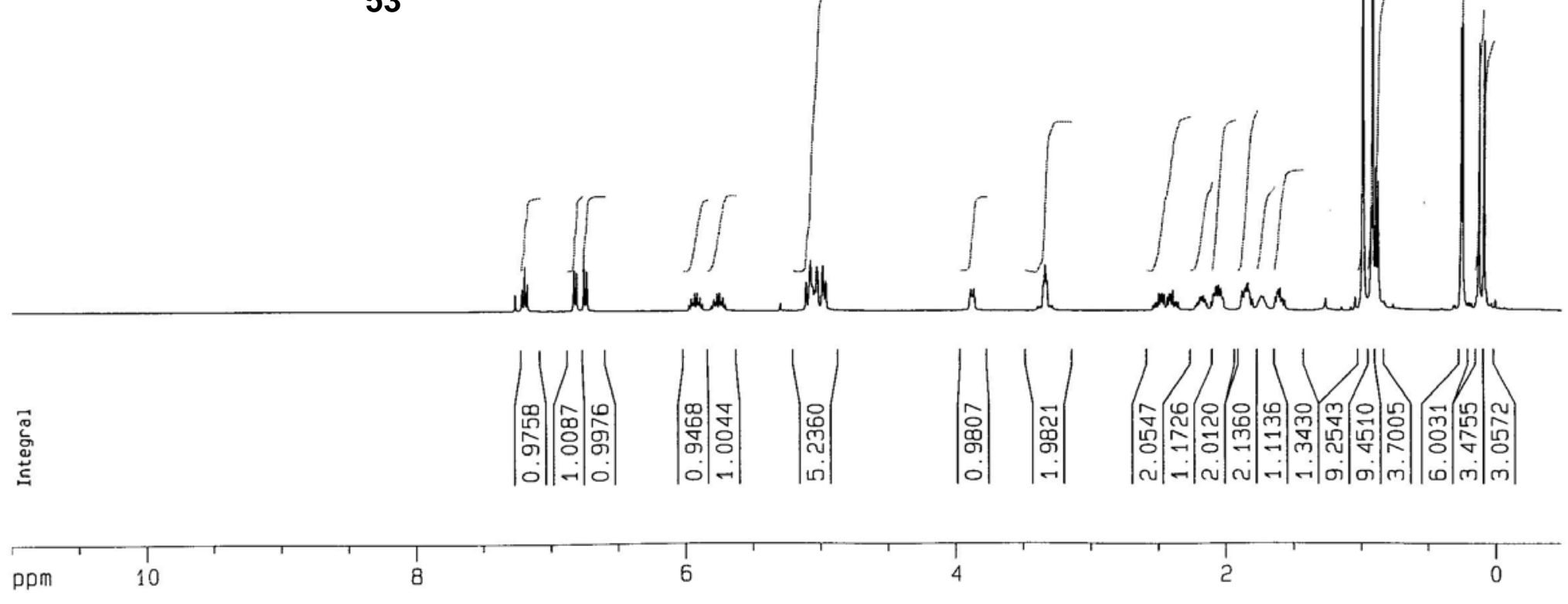




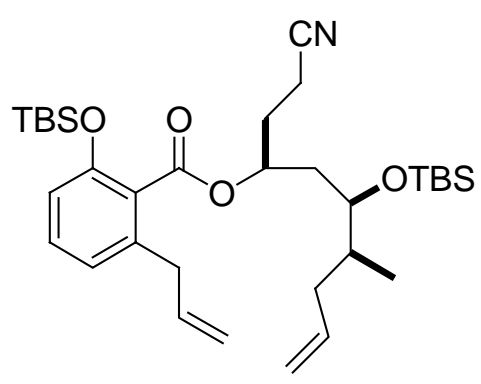

53

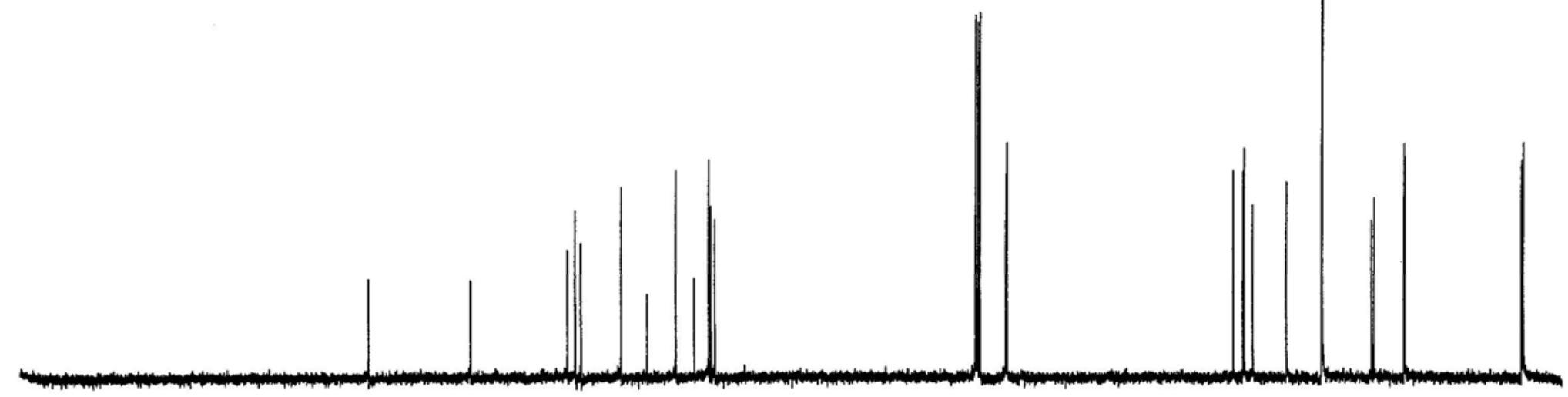




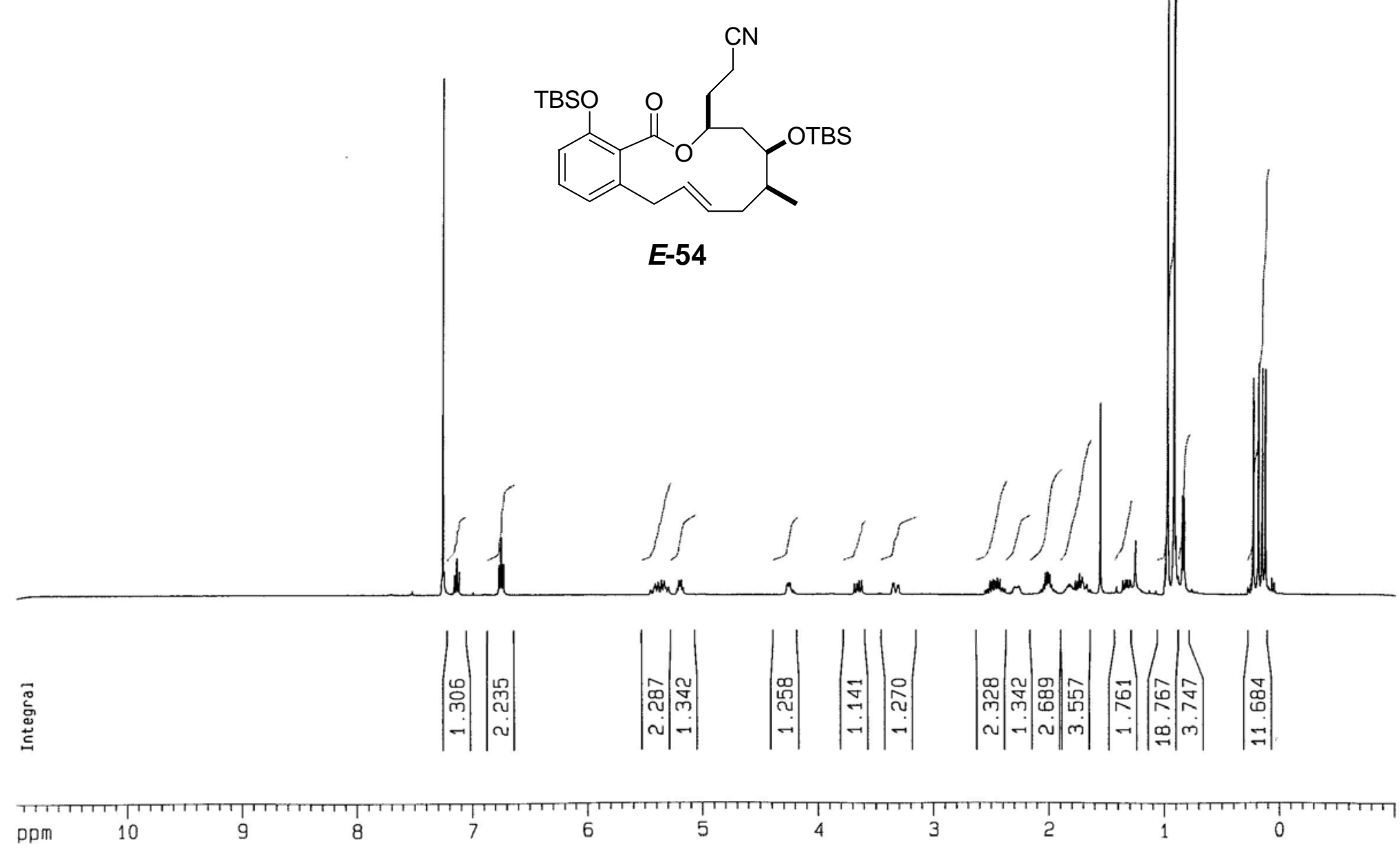




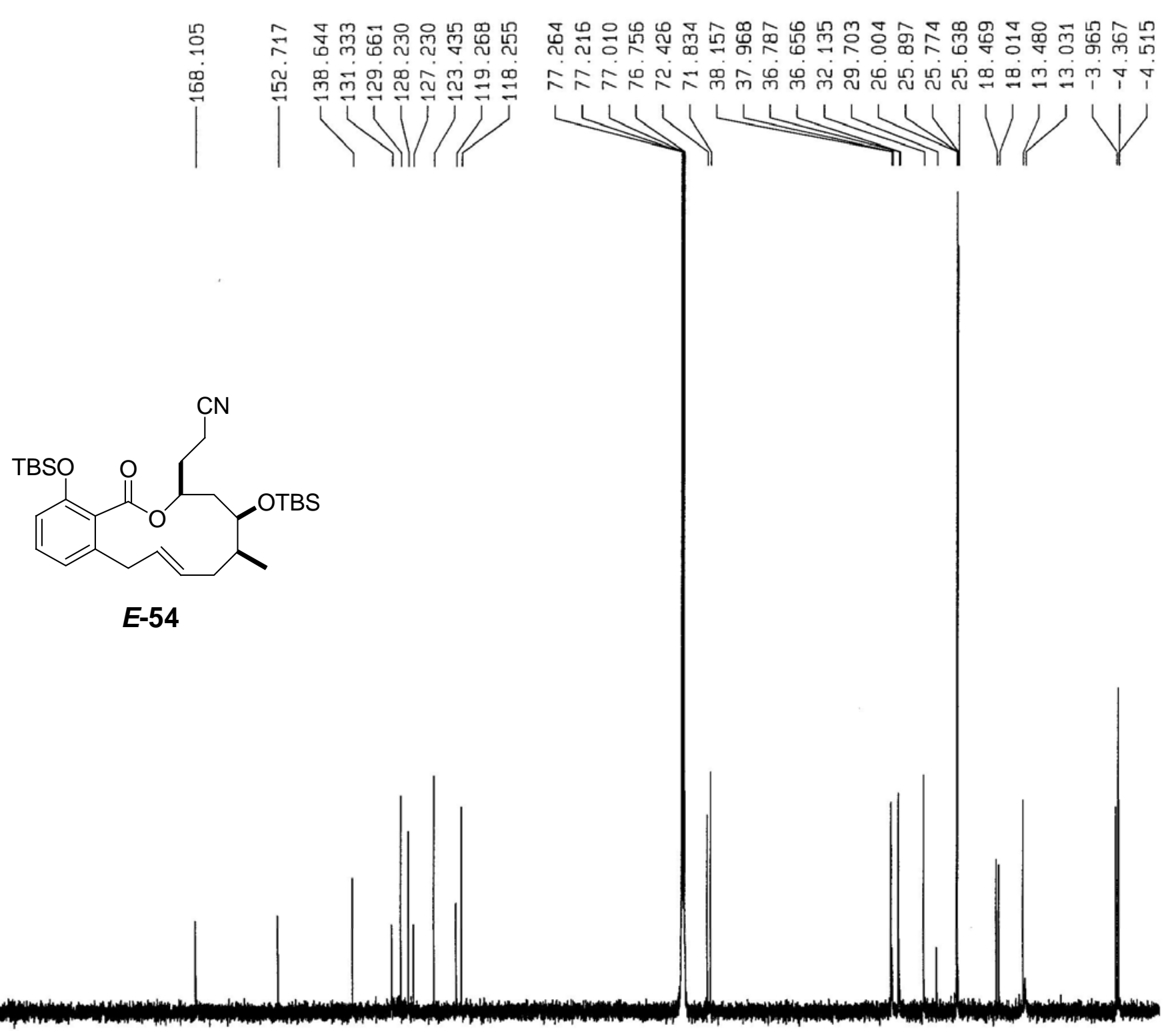




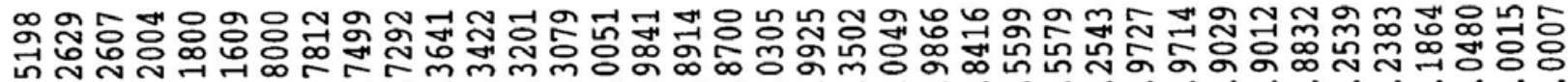

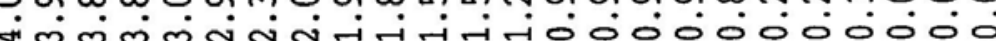

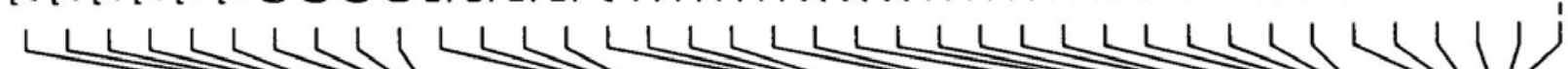
กา 11

71

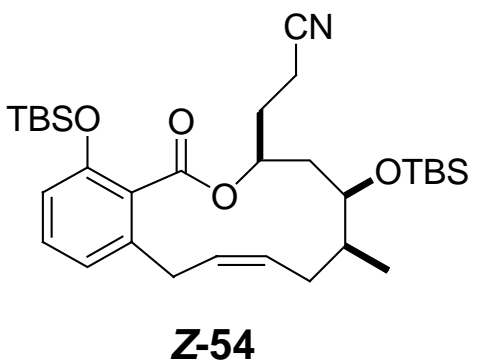

怨

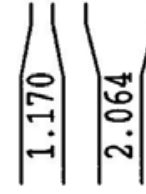

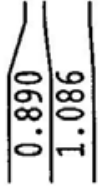

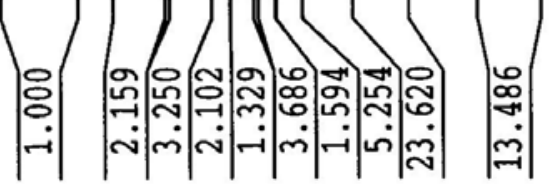




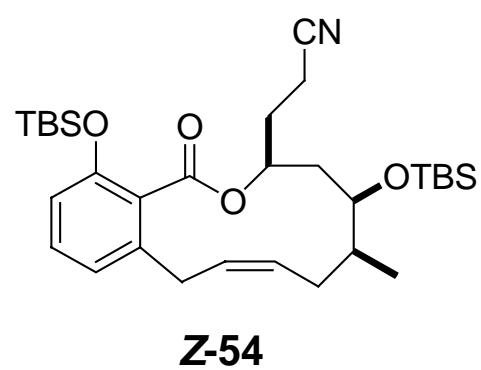

